\title{
Nanobodies as new tools for studying large cargo transport and lamina organization
}

\author{
Dissertation \\ For the award of the degree \\ "Doctor rerum naturalium" \\ within the Molecular Biology of Cells program \\ of the Georg-August-Universität Göttingen \\ Faculty of Biology
}

Submitted by

Myroslav Gebura

Born in

Kalush, Ukraine

Göttingen, August 2017 


\section{Members of the Thesis Committee}

Prof. Dr. Dirk Goerlich

(Supervisor and referee)

Prof. Dr. Juergen Wienands

Prof. Dr. Markus Bohnsack
Max Planck Institute for Biophysical Chemistry

Department of Cellular Logistics

Georg August University Goettingen

Department of Cellular and Molecular

Immunology

Georg August University Goettingen

Department of Biochemistry I

\section{Members of the extended Thesis Committee}

Prof. Dr. Henning Urlaub

Prof. Dr. Wolfgang Wintermeyer

Prof. Dr. Detlef Doenecke

Department of Biochemistry I

Bioanalytical Mass Spectrometry Group

Max Planck Institute for Biophysical Chemistry

Department of Physical Biochemistry

Georg August University Goettingen

Date of the oral examination: 9.10.2017 
I hereby declare that I completed my thesis entitled "Nanobodies as new tools for studying large cargo transport and lamina organization" independently and with no other aids or sources then quoted.

This dissertation has not been submitted elsewhere for any academic award of qualification Myroslav Gebura

Göttingen, August 2017 
"God is in the details"

- Proverb - 


\section{Table of content}

1 Summary ...................................................................................................................................... 11

2 Introduction ........................................................................................................................... 12

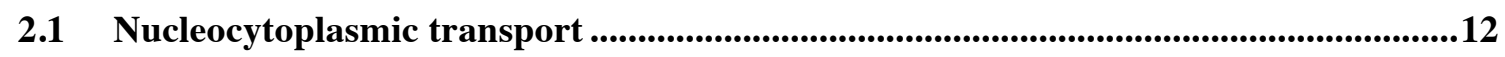

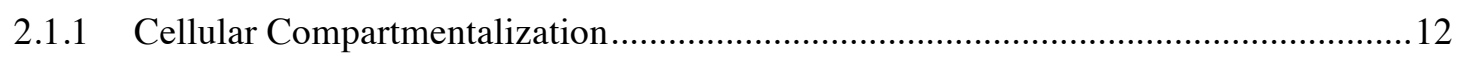

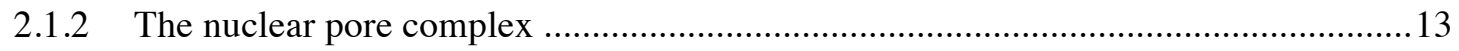

2.1.3 FG repeats form NPC permeability barrier …..........................................................

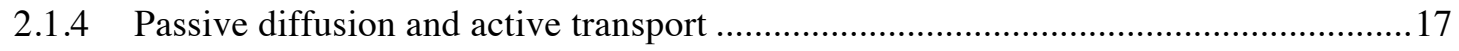

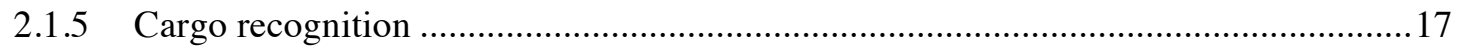

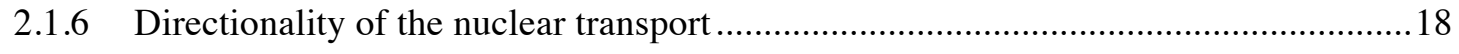

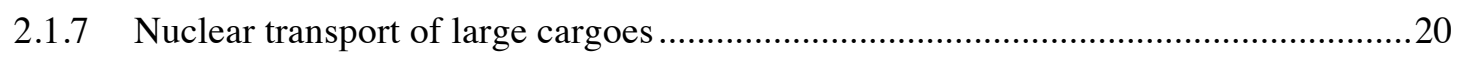

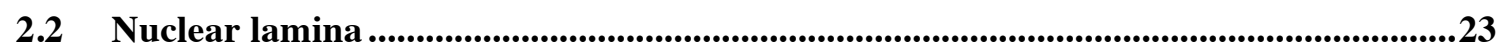

2.2.1 Lamins are building blocks of the nuclear lamina ...................................................23

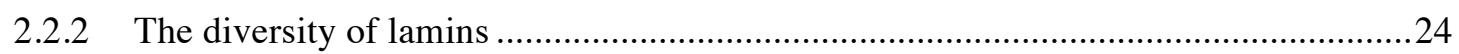

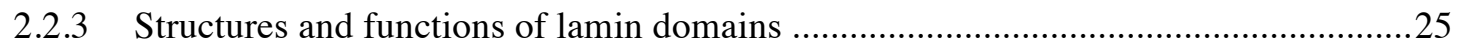

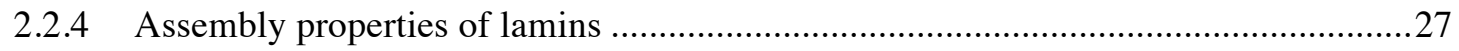

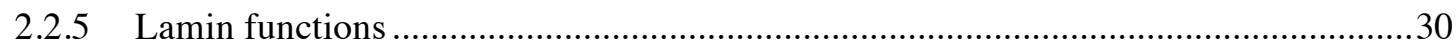

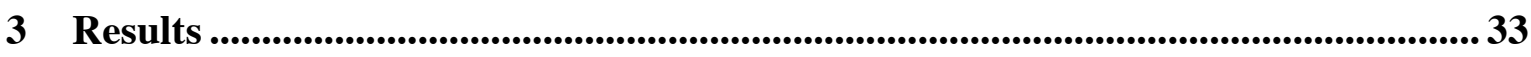

3.1 Generation of anti-ribosomal nanobodies en route to transport competent ribosomal

subunits ...............................................................................................................................................................34

3.1.1 Purification of 30S and 50S ribosomes from Thermus thermophilus ..........................34

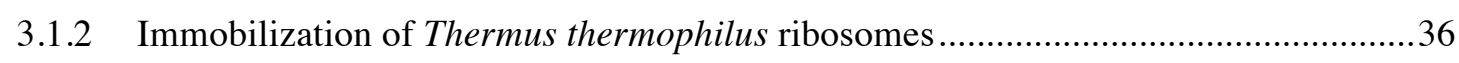

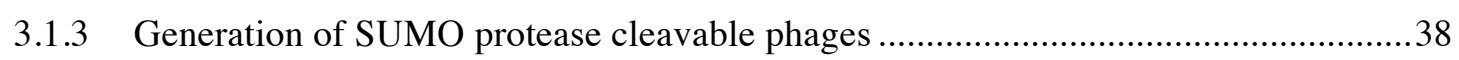

3.1.4 Selection of anti-ribosomal nanobodies and their characterization .............................4

3.1.5 Identification of binding partners of nanobodies by cross-linking ..............................4

3.1.6 Identification of rRNA-binding nanobody by CRAC …............................................48

3.1.7 Ribosomal subunit transport in FG-hydrogel permeation assay .................................51

3.2 Generation of nanobodies against lamin isoformes from Xenopus laevis......................53

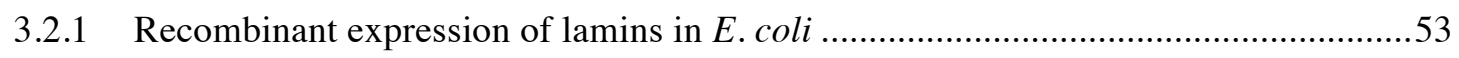

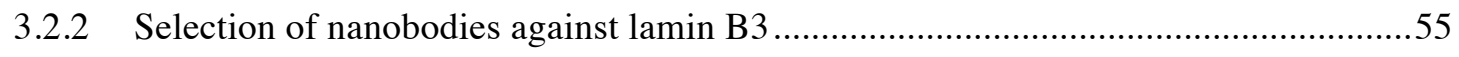

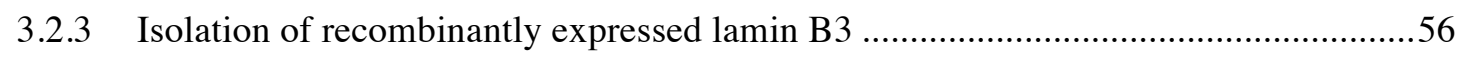

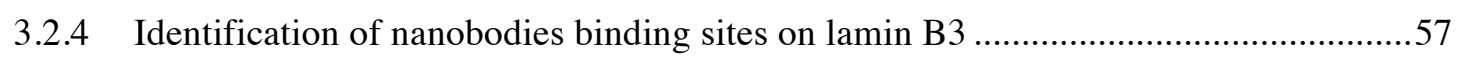

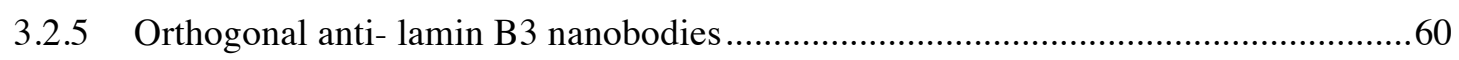

3.2.6 Specificity of selected orthogonal nanobodies against different lamin isoforms .........61

3.2.7 Native isolation of lamin isoforms from Xenopus laevis eggs ....................................64 
4 Discussion ............................................................................................................................. 71

4.1 SUMO protease cleavable phage M13 …...............................................................................71

4.2 Nanobodies against $T$ th ribosomes and the rRNA binding nanobody ...........................73

4.3 Mapping of nanobodies binding sites on large antigens ..................................................74

4.4 Nanobodies as tools to study transport of ribosomal subunits .......................................75

4.5 Recombinant expression of soluble lamins .....................................................................

4.6 Anti-lamin B3 nanobodies and their applications .........................................................

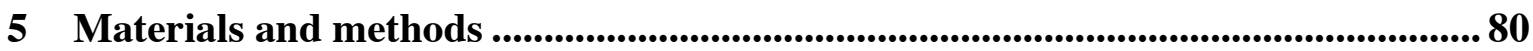

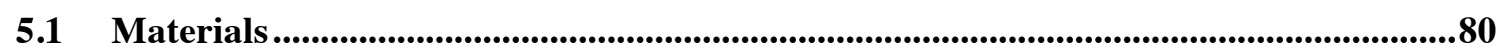

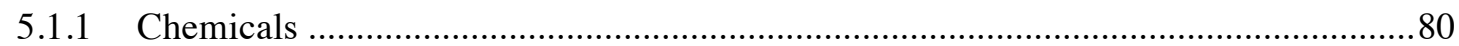

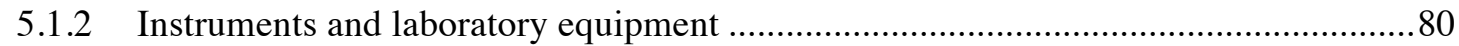

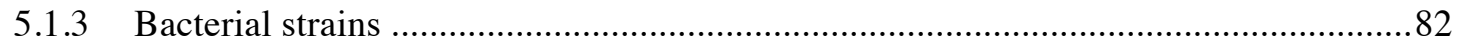

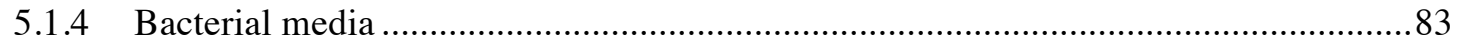

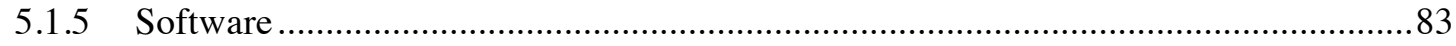

5.2 Standard methods in molecular biology ...............................................................................84

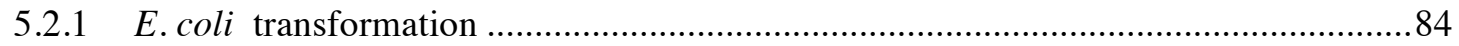

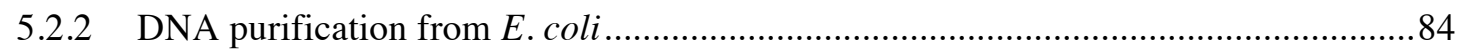

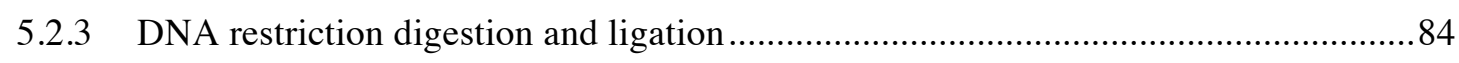

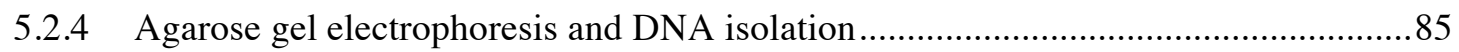

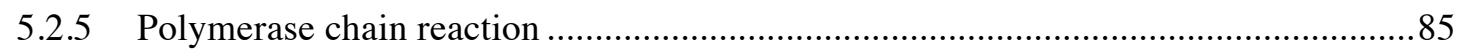

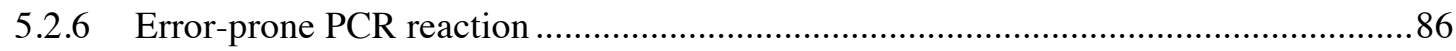

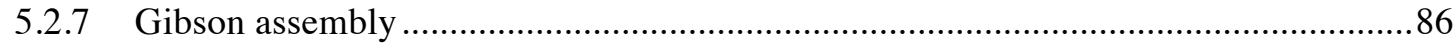

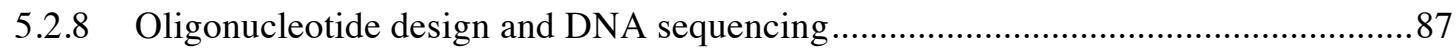

5.3 Protein expression and purification .......................................................................................87

5.3.1 Recombinant protein expression in E. coli ..................................................................

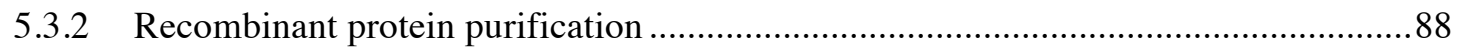

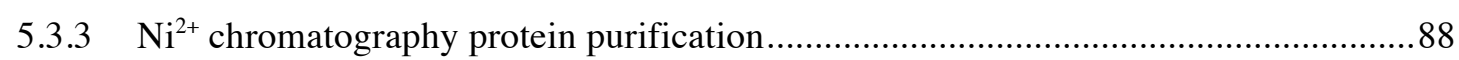

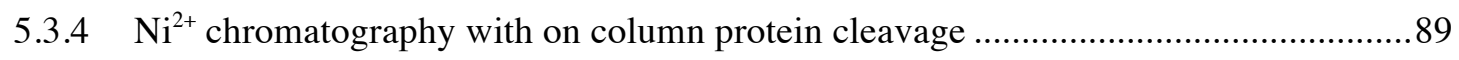

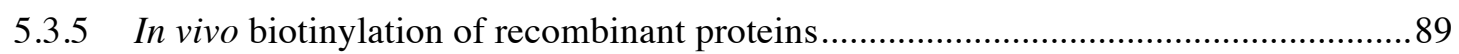

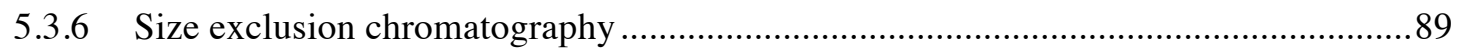

5.3.7 Purification of 30S and 50S Thermus thermophilus ribosomal subunits ....................90

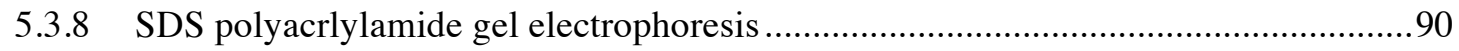

5.3.9 Maleimide-flurophore conjugation to proteins with free -SH groups ........................91

5.3.10 Biotinylation of 70S Thermus thermophilus ribosomes ............................................91

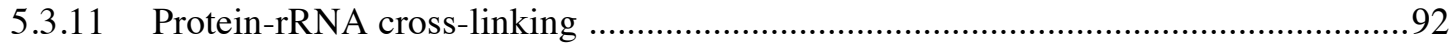




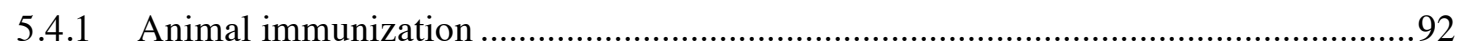

5.4.2 Lymphocyte isolation and total RNA preparation ....................................................92

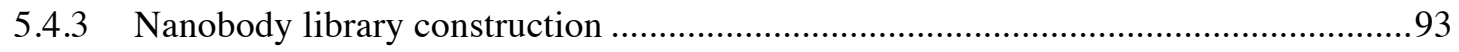

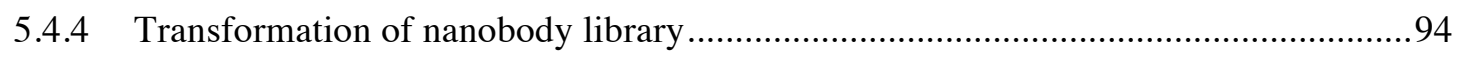

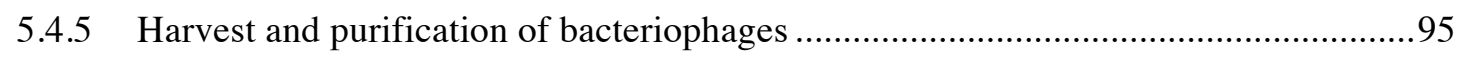

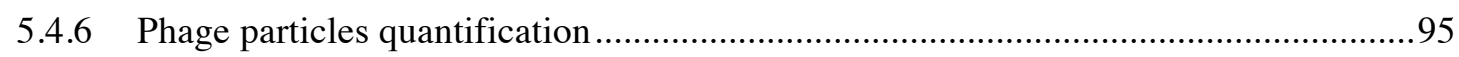

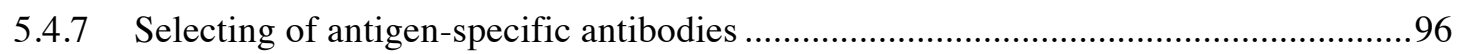

5.4.8 Re-cloning of bio-panned nanobodies into an expression vector .................................97

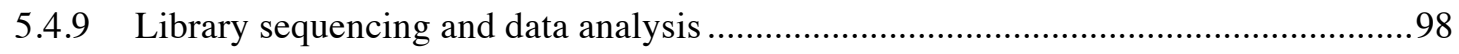

5.5 In vitro methods using Xenopus laevis egg extract ........................................................98

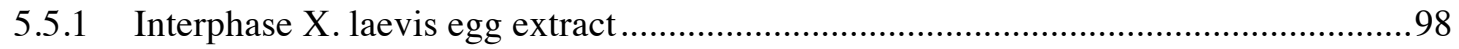

5.5.2 Protein isolation from Xenopus laevis egg extract .....................................................99

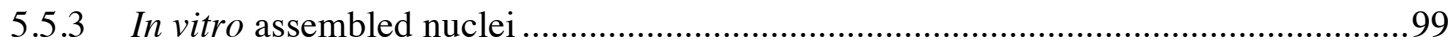

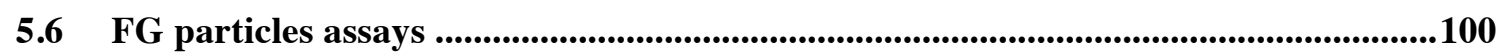

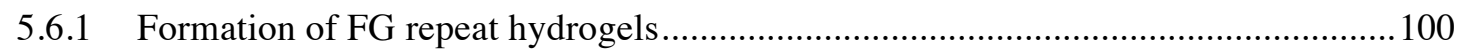

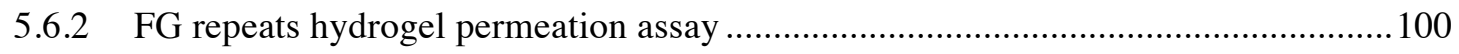

5.7 Cell cultures and immunostaining .......................................................................101

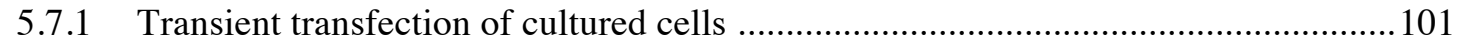

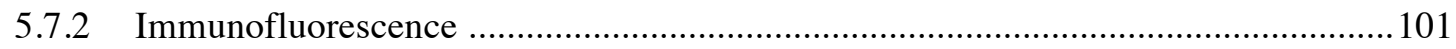

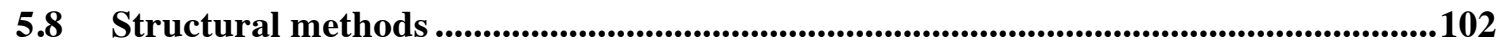

5.8.1 Cross-linking mass spectrometry for protein identification ......................................102

5.8.2 Nanobody epitope mapping by cross-linking …….................................................102

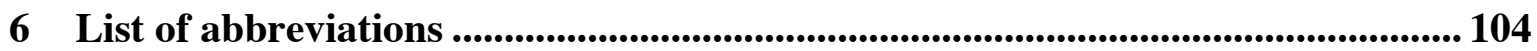

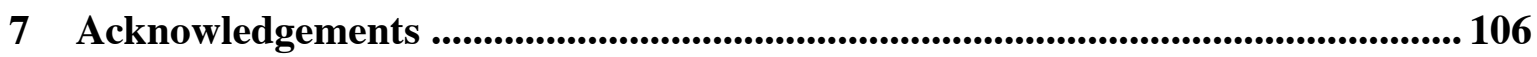

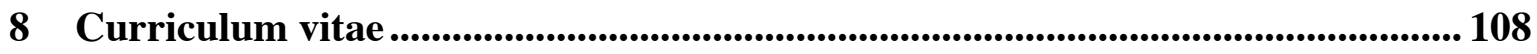

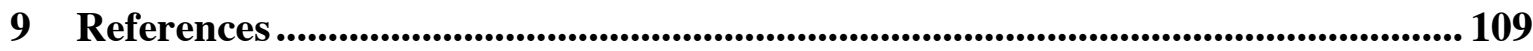




\section{Summary}

Nanobodies are the smallest recombinantly expressed antibody fragments, which show outstanding performance in numerous applications. In this work, we set out to generate nanobodies as tools to study nucleo-cytoplasmic transport of large cargoes, such as ribosomes. We also generated a set of nanobodies targeting the $X$. laevis lamina.

To meet these objectives we have optimised phage-display and designed an M13 phage that presents a nanobody library in SUMO-protease cleavable form. This allows a very specific phage-elution from immobilised antigens, namely by adding the SUMO-protease in a mild physiological buffer, which releases the phages from the still antigen-bound nanobody.

Using our newly developed SUMO-cleavable phage M13 we have successfully selected and characterized six orthogonal nanobodies against ribosomes from Thermus thermophilus. This includes the very first nucleic acid-binding nanobody targeting the Asite of the large ribosomal subunit.

So far, all attempts to produce lamins in bacteria lead to insoluble aggregates. We now discovered, however, that certain solubility-enhancing tags combined with appropriate protease cleavage modules allow E. coli to produce lamins in a soluble and correctly folded form. This not only established that lamins do not require any specialised eukaryotic chaperones for folding, but also allowed to isolate a set of orthogonal anti-lamin B3 nanobodies. We show that anti-lamin nanobodies perform excellently in purifying native protein complexes as well as in imaging. 


\section{Introduction}

\subsection{Nucleocytoplasmic transport}

\subsubsection{Cellular Compartmentalization}

The nucleus is a hallmark of every eukaryotic cell and the presence of a nucleus has introduces many evolutionary benefits to eukaryotes. Arguably, one of the most obvious advantages of the nuclear compartmentalization is the enclosure and protection of the cell's genomic information. Such a segregation allows for the regulation of the access of unwanted elements e. g. aberrant proteins, free radicals or even viruses, which otherwise might have deleterious effects on DNA.

An even more striking evolutionary advantage of the nucleus is a separation of the transcription machinery from the translation apparatus. Alternative mRNA splicing, which takes place in the nucleus, offers a means to multiply the number of protein variants that can be obtained from a single gene. Due to the nucleus, the size of the genome itself could be increased manifold in comparison to prokaryotes, thus it could store more coding and regulatory DNA sequences. Protein translation, on the other hand, is decoupled from the transcription and takes place in the cytoplasm, which allows for effectiveness and a high fidelity of gene expression.

This order of events necessitates an effective and regulated molecular crosstalk between the nucleus and the cytoplasm. In this sense, all the proteins that carry out functions in the nucleus e. g. DNA maintenance, regulation of gene expression, structural proteins have to be delivered to the nucleus. Conversely, mRNA, tRNA, mature ribosomal subunits must constantly flux to their destination compartment - the cytoplasm.

Importantly, the physical boundaries of the nucleus are defined by a double layer membrane, which is not permeable for most of water soluble molecules in the cell. How could the cell then ensure transport of molecules between the cytoplasm and the nucleus? To tackle this fundamental dilemma the cell has originated a specialized macromolecular protein assembly termed the nuclear pore complex (NPC) (Watson, 1954). 


\subsubsection{The nuclear pore complex}

The nuclear pore complex is the sole site for nuclear passage, it creates a diffusion barrier for certain molecules and is, simultaneously, highly permissive for others Figure 2.1.

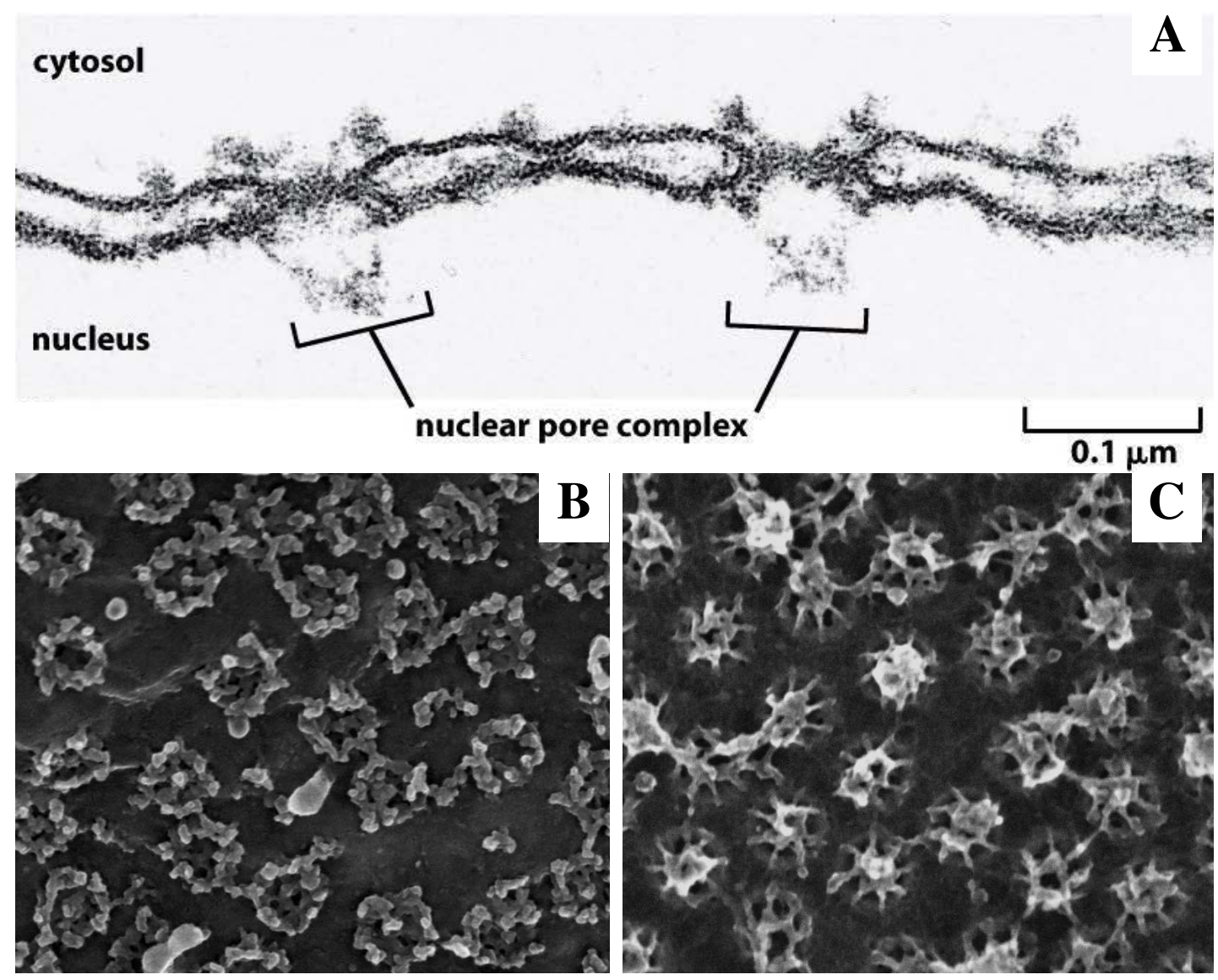

Figure 2.1 The nuclear pore complex arrangements in the nuclear membrane. (A) A crosssection of nuclear membrane harboring NPCs. A side view of the NPCs represents the continuity of outer and inner leaflets of the membrane. The structural components, as well as the nuclear basket of the NPC can be assessed. (B) The cytoplasmic side of the nuclear membrane from a frog oocyte obtained by scanning electron microscopy. (C) Face-on representation of NPCs nuclear side obtained by negative stain electron microscopy. Note the eight-fold symmetry of the nuclear basket. The picture A was reproduced from Alberts et al. 2002. Pictures B and C were kindly provided by Dr. Volker Cordes (Max Planck Institute for Biophysical Chemistry).

Since the discovery of the NPC (Gallan H.G., 1950) there were numerous scientific studies describing the composition and functions of this large multi-protein assembly. One of the very first observations was that the NPC spans through the membrane, fusing the outer and the inner leaflets of the nuclear membrane. The outer nuclear membrane creates a continuum with the endoplasmatic reticulum, while the inner nuclear membrane is restrictive and compartment specific. Later on, the eight-fold symmetry of NPCs was observed (Watson, 1959) and the fact that the NPC is asymmetric: the cytoplasmic side of 
the NPC is equipped with eight highly flexible domains also known as filaments, whereas the nuclear side has a basket-like structure. With the advancements in biochemistry the size of the NPC could be estimated, which ranges from $66 \mathrm{MDa}$ in yeast to an impressive 125 MDa in vertebrates (Reichelt et al., 1990; Rout and Blobel, 1993). The most recent technological improvements in the field of cryo-electron microscopy have further shed light onto the NPC architecture. By means of cryo-electron tomography the NPC structure from Xenopus laevis was determined at resolution up to $20 \AA$ (Eibauer et al., 2015)Figure 2.2 .
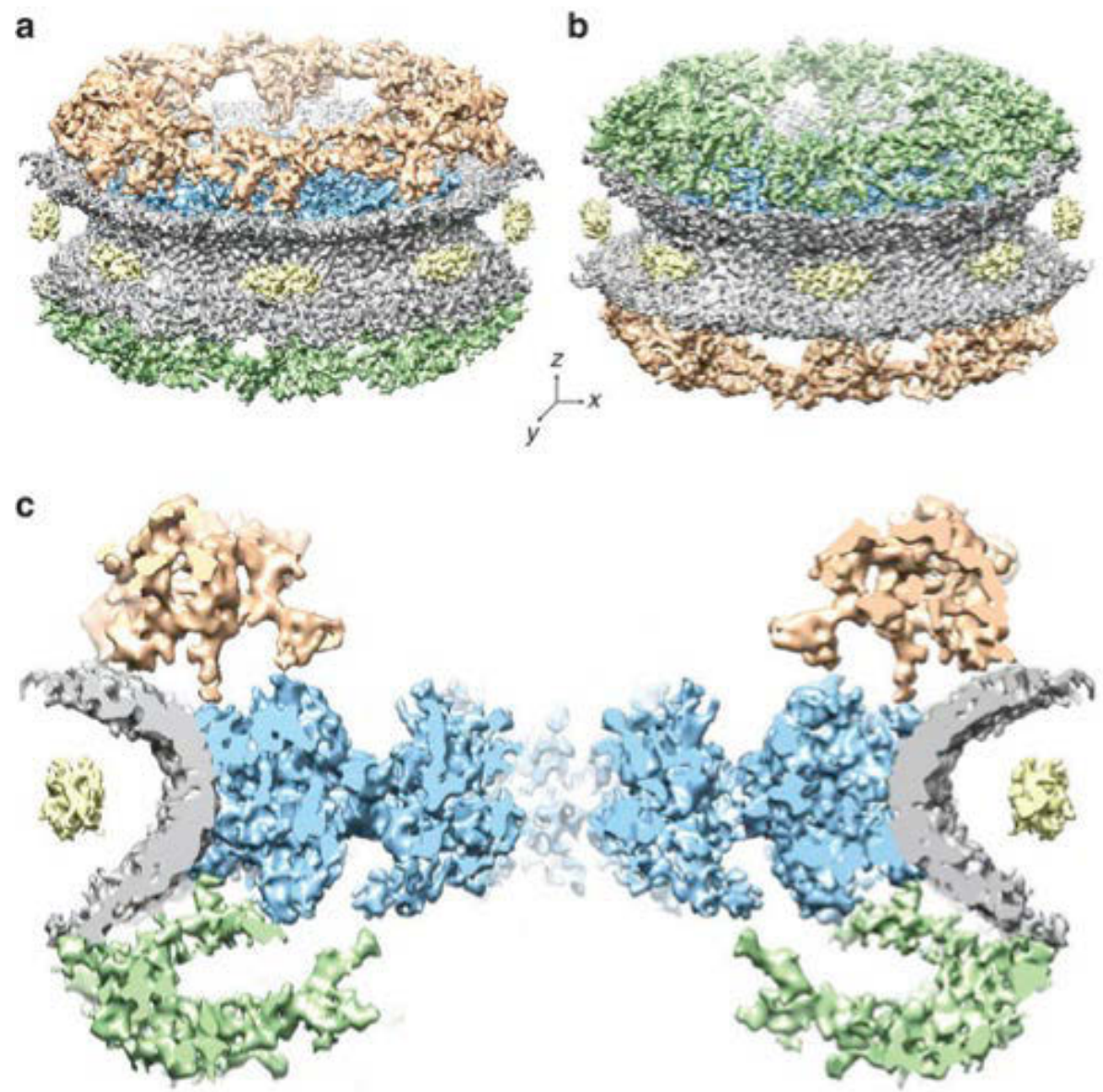

Figure 2.2 The structure of Xenopus laevis NPC. (A) Surface representation of the cytoplasmic ring in golden color. (B) The nucleoplasm ring is represented in green. (C) The section through the NPC. The pictures was adapted from Eibauer et al. 2015.

Although the NPC has a large molecular mass, it is composed only of 30 individual nucleoporins (Nups), which are present in multiple copies (Ori et al., 2013; Rout et al., 2000) Figure 2.3. All Nups can be divided into two categories: structural Nups, which compose all rigid structural elements, and Nups, which contain disordered elements, filling in the central pore of the NPC (Schwartz, 2005). 


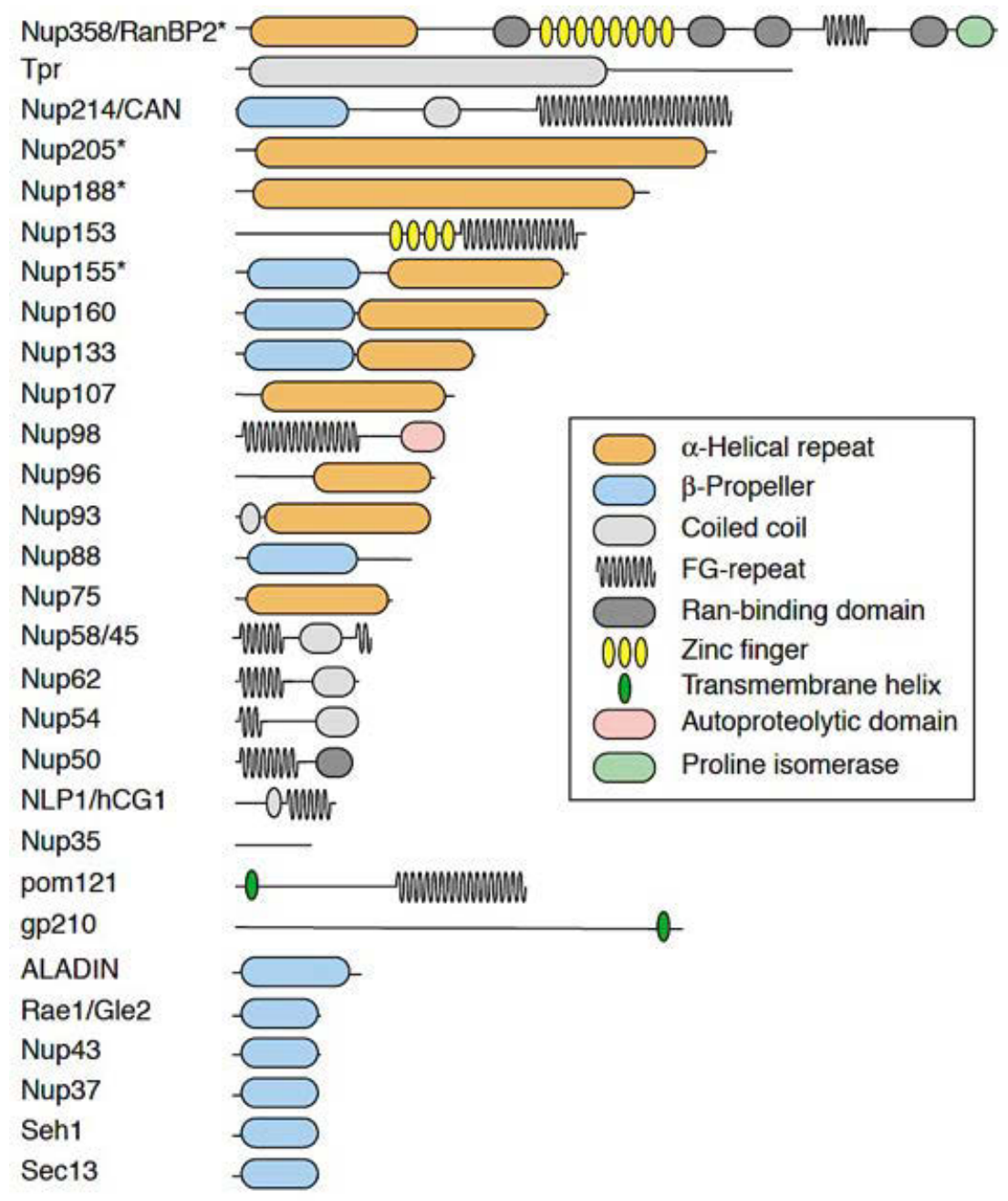

Figure 2.3 Schematic representation of vertebrate Nups domains. An asterisk represents $\alpha$ helical repeats predicted with high confidence (Schwartz 2005).

Protein members of the intrinsically disordered group are rich in phenylalanine-glycine repeats, also known as FG-repeats (Wente et al., 1992). Their importance lies in the fact that they represent the interaction sites with molecular complexes that are transported through the NPC, thus creating a permeability barrier (Frey and Görlich, 2007; Hülsmann et al., 2012; Ribbeck and Görlich, 2001).

\subsubsection{FG repeats form NPC permeability barrier}

The very first FG containing nucleoporins (FG-Nups), Nsp1 and Nup1, were identified in yeasts (Hurt, 1988). Since their discovery numerous Nups were also reported to contain FG repeats and they were found across all the eukaryotic kingdoms (H. B. Schmidt, 2015). Between $12 \%$ and $20 \%$ of all the Nups were estimated to contain FG repeats (Iovine et al., 1995), which together accounts for approximately $13 \mathrm{MDa}$ of all the protein mass in the NPC (Ori et al., 2013). FG-Nups reside in the parts of the NPC exposed to the peripheral 
surface and the innermost central channel, which speaks for their involvement in the barrier formation.

FG-Nups are intrinsically disordered and the unfolded structure of FG-Nups originates from the repetitive FG units themselves, which are flanked by hydrophilic spacers (Denning et al., 2003). Unstructured FG protein domains are composed of up to 50 single FG dipeptide motifs. Additionally, these hydrophobic clusters can be formed either by FG, VFG, and GLFG (Webster et al., 2014). As FG motifs can be formed by different residues, their resulting physical properties also can differ significantly (Xu and Powers, 2013).

Despite being unstructured proteins, FG-Nups have well defined interactions, which can be further grouped into three categories. First of all, the FG-Nups interact with the structural components of the NPC (Andersen et al., 2013), what allows their effective incorporation into the NPC. The integration of the FG-Nups into the NPC is believed to be facilitated by their non-FG domains (Bailer et al., 1998) and when these conserved domains are deleted the FG-Nups become mislocalized (Zeitler and Weis, 2004).

Secondly, FG-Nups engage into intra- and intermolecular interactions, creating a continuous mesh-work. This mesh-work was demonstrated to be a water-containing polymer (hydrogel) created by physically linked FG repeats (Frey and Gorlich, 2009; Frey and Görlich, 2007; Frey et al., 2006). Reasonably, FG-Nups are instrumental for the formation of the selective gate of the NPC. Even when FG-Nups are expressed recombinantly they undergo self-assembly to form hydrogels that resemble properties of the permeation barrier of the NPC (Schmidt and Görlich, 2015).

Finaly, the FG-Nups ability to form hydrogels is integral to the process of cargo translocation, which represents the third group of interactions (Bayliss et al., 1999). These hydrogels formed by FG-Nups contribute both to the permeability barrier and to the passive and active transport of cargoes via the NPC. On one hand, FG hydrogels allow a rapid passage of small molecules and metabolites, on the other hand, they become increasingly restrictive when the size of a molecule approaches $40 \mathrm{kDa}$ (Bonner, 1975). At first glance, this finding might be puzzling as it raises a question how cells transport large molecules through the NPC. Eukaryotic cells have liberated the passage of larger molecules by designing an elegant mechanism, which relies on nuclear transport receptors (NTRs). 


\subsubsection{Passive diffusion and active transport}

There are two modes of nuclear transport: a passive diffusion and an active transport. Several molecular characteristics contribute greatly to the way a molecule will be transported via the NPC. The foremost feature that allows passive diffusion of a molecule through the NPC is its size. Smaller organic substances e. g. ethanol, glycerol, or steroids can basically permeate the nuclear membrane freely. Molecules with a nominal size below $\leq 5 \mathrm{~nm}$ can also diffuse into the nucleus via NPCs. Although there are numerous molecules that fall into the first category and can freely enter the nucleus, larger ones, conversely, are strictly excluded from nucleus. An appropriate NTR must be recruited for a larger molecule to become transport competent.

Transport receptors represent a protein family of soluble proteins, with 21 members in mammals and 14 in yeasts (Fornerod et al., 1997a; Görlich et al., 1997). NTRs are large proteins, which range in size from 90 to $150 \mathrm{kDa}$. Depending on the directionality of a cargo transport, NTRs can be classified as importins (e. g. importin $\beta$ ), exportins (e. g. CRM1), or bi-directional NTRs (e. g. Imp13) (Görlich et al., 1997; Mingot et al., 2001). All NTRs are sequence-related to importin $\beta(\operatorname{Imp} \beta)$ and share similar structural elements, so-called HEAT repeats. Every HEAT repeat consists of two anti-parallel $\alpha$-helices linked by flexible inter-unit loops (Groves et al., 1999). NTRs are made of about 18-20 HEAT repeats arranged into two orthogonal arches with a slight clockwise tilt giving rise to a solenoid-like structure (Chook and Blobel, 1999). Such a molecular architecture appears to be inherently flexible allowing NTRs to recognize and release their cargos (Conti et al., 2006). Linear arrangement of HEAT repeats creates a large surface allowing NTRs to interact with their cargoes and RanGTP, which represent the most prominent common features of all NTRs. The first step in a cargo transport is its recognition, thus it is worth discussing this mechanism more in detail.

\subsubsection{Cargo recognition}

The primary task of NTRs is to recognize their cargo among all the other cellular proteins. This NTRs function is achieved by the presence of nuclear localization or nuclear export signals on cargoes (abbreviated as NLS and NES, respectively). Proteins destined to be in the nucleus contain NLS, which consist of a mono- or bipartite stretch of basic amino acids. The monopartite NLS are represented by the one present on the well studied simian 
virus 40 (SV40) large T antigen (Kalderon et al., 1984), while the bipartite NLS can be exemplified by the one present on nucleoplasmin (Dingwall and Laskey, 1991).

The best-known NESs, on the other hand, are the PKI NES (Wen et al., 1995) and the NES from HIV virus protein Rev (Fischer et al., 1995). Importantly, both NES types are recognized by CRM1 (Fornerod et al., 1997b). These NES are short peptides, which are comprised out of five hydrophobic consensus resides flanked by short spacer residues (Güttler et al., 2010).

There are, however, situations where NTRs cannot bind their cargo directly. In such a case, an NTR must bind an adaptor molecule. The most studies adapter molecule is importin $\alpha$ $(\operatorname{Imp} \alpha)$ (Görlich et al., 1994).The function of $\operatorname{Imp} \alpha$ is to recognize the actual NLS and to bind the cargo-molecule together with $\operatorname{Imp} \beta$. The $\operatorname{Imp} \beta$ is a true transport receptor and accounts for the translocation of the trimetric complex to the nuclear side of the NPC. The accessory protein Imp $\alpha$ is transported back to the cytoplasm by CAS exportin, whose only function is Imp $\alpha$ re-export (Kutay et al., 1997).

\subsubsection{Directionality of the nuclear transport}

The presence of NTRs alone is not sufficient for a cargo translocation. Thus, the cell has developed a neat mechanism to control the directionality of nuclear transport via the RanGTP system. Ran is a member of a Ras-protein family. Being a GTPase, it can switch between two states a GTP- and a GDP- state. The RanGTP form is 1000 fold more abundant in the nucleus than in the cytoplasm (Görlich et al., 2003), RanGDP, on the contrary, is more enriched in the cytoplasm. Such as steep gradient of RanGTP is achieved by specific molecular machinery that regenerates RanGTP in the nucleus. RCC1 is a Ranspecific nucleotide exchange factor, which is bound to chromatin and present exclusively in the nucleus (Bischoff and Ponstingl, 1995). Thus, RCC1 ensures a high nuclear level of RanGTP. When RanGTP is in the cytoplasm two accessory proteins activate the GTPase function of Ran. RanGAP (facilitated by RanBP1) stimulates GTP hydrolysis by Ran up to $10^{5}$ fold (Bischoff et al., 1995). Both RanGAP and RanBP1 are strictly cytoplasmic proteins, what ensures GTP hydrolysis specifically in the cytoplasm.

Finally, to replenish nuclear RanGTP pool, RanGDP form has to be transported back to the nucleus. This task is carried out by a dedicated NTR called NTF2, which is a $15 \mathrm{kDa}$ transport receptor. It forms homodimers and specifically recognizes two molecules of 
RanGDP and reimports them back into the nucleus (Ribbeck et al., 1998). The final result of the above described mechanisms is a RanGTP gradient across the nuclear envelope, with high nuclear and low cytoplasmic RanGTP concentrations, where the steep RanGTP gradient fuels the active transport (Görlich et al., 1996) Figure 2.4.

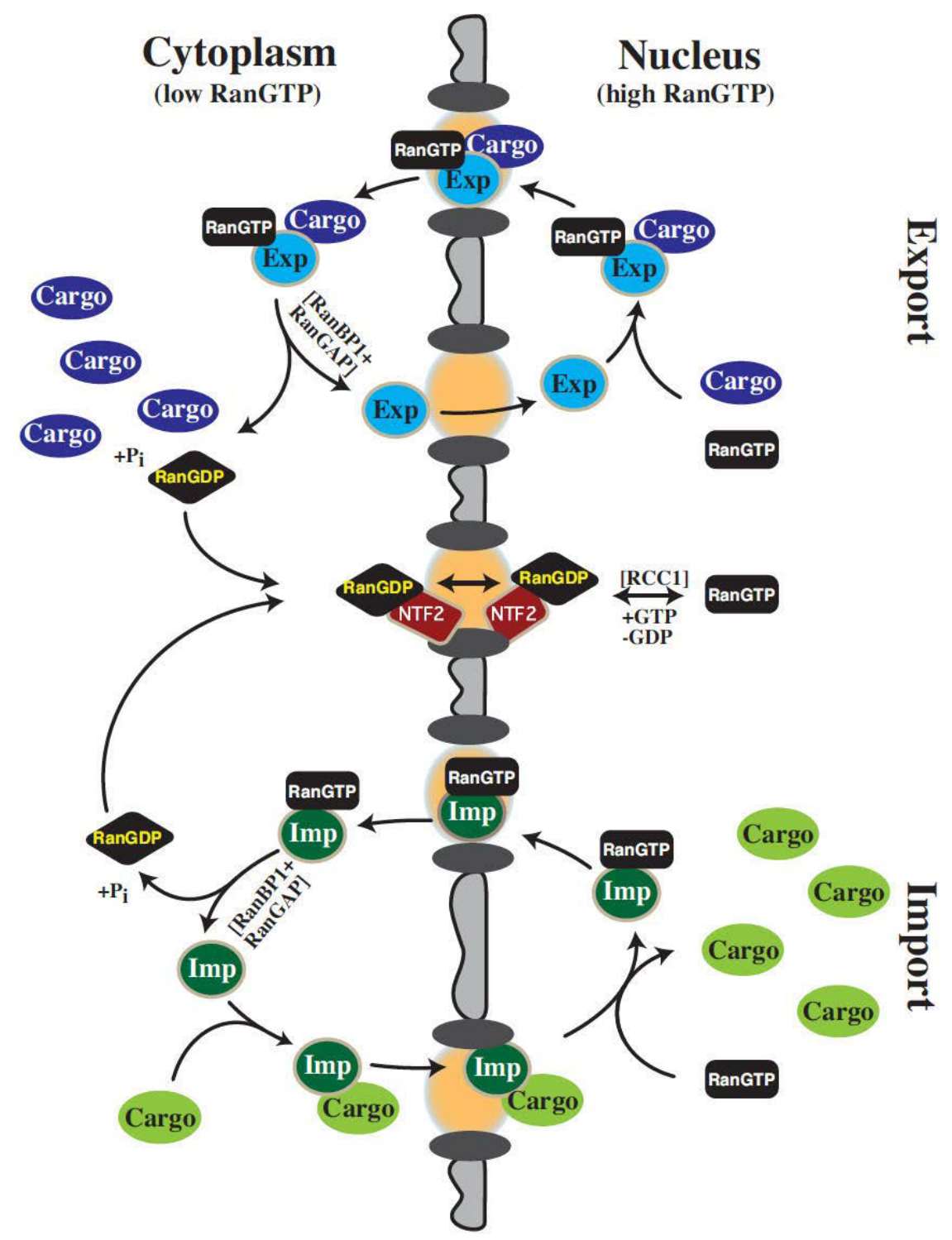

Figure 2.4 Transport cycles of importins and exportins with the relation to the RanGTP system. Modified from Görlich \& Kutay 1999.

Although the nuclear transport per se is energy independent, NTRs respond to the RanGTP gradient by loading/unloading their cargo molecules (Englmeier et al., 1999). Both exportins and importins have the propensity to bind RanGTP. This binding, however, induces different conformational changes in exportins and importins. 
Importins bind their cargo on the cytoplasmic side at low concentration of RanGTP. An importin together with its cargo traverses through the nuclear membrane and encounters a high RanGTP concentration in the nucleus. The high RanGTP was proposed to facilitate the release of an importin/cargo complex at the nuclear side of the NPC, thus terminating a cargo passage (Görlich et al., 1996). Importins bind RanGTP, which displaces the cargo from the NTR, and an importin/RanGTP complex travels back to the cytoplasm. As discussed previously, Ran-bound GTP is hydrolyzed in the cytoplasm in the presence or RanBP1 and RanGAP and this hydrolysis causes the RanGDP to dissociate, setting the importin free and ready for the next translocation cycle (Bischoff and Görlich, 1997).

Exportins, on the other hand, harvest the RanGTP cycle quite in the opposite way. Exportins bind cargo together with RanGTP in the nucleus. Importantly, such a trimetric complex formation it highly cooperative: RanGTP increases the affinity of exportins to bind their cargo a 1000 -fold, and vice versa. A trimetric complex consisting of an exportin/cargo/RanGTP is transported into the cytoplasm. Already mentioned RanBP1 and RanGAP facilitate the complex dissociation by stimulating GTP hydrolysis by Ran (Kutay et al., 1997; Monecke et al., 2013; Petosa et al., 2004).

In summary, both importins and exportins can bind RanGTP, but RanGTP has an opposite effect on the export and import of molecules. In the case of importins RanGTP binding is mutually exclusive with the presence of the cargo, as their binding sites overlap. Thus when RanGTP binds an importin, the latter's cargo is dislodged. Binding to exportins, on the other hand, facilitates the cargo binding. To summarize, the directionality of nuclear transport is determined by Ran, governing cargo loading/unloading onto NTRs.

\subsubsection{Nuclear transport of large cargoes}

Nuclear passive diffusion occurs when size of a molecule does not exceed 20-40 kDa limit. Larger molecules are transported by an active involvement of transport receptors. However, NTRs alone do not dictate the properties and the rate of NPC passage. On the contrary, it was established that also the cargo itself influences the translocation rates. Particularly, not only the size of a cargo, but also the ratio of NTRs to the cargo influences the speed of translocation (Ribbeck and Görlich, 2002).

NTRs facilitate active transport of molecules with a wide range of sizes. Molecules as small as $40 \mathrm{kDa}$ can be transported by an NTR. On the other side of the spectrum are the 
largest cellular cargoes such as ribosomal subunits with their nominal size of 2,6 MDa. Evidences from biochemical and genetic experiments suggest that ribosomes are exported out of the nucleus as separate subunits rather then a single entity. It was also reported that both the small and the large ribosomal subunit rely on CRM1, supported by the findings that a potent inhibitor of CRM1 leptomycin B inhibits export of both subunits (Ho et al., 2000; Moy and Silver, 2002). Before and during the export ribosomal subunits undergo several alterations in order to become transport competent. Several studies, by means of genetics, revealed the complexity of factors involved in ribosomal biogenesis (Hurt et al., 1999; Moy and Silver, 2002; Stage-Zimmermann et al., 2000). Arguably, ribosomal biogenesis and its transport are very much intertwined processes, both contributing to the proper maturation of ribosomes. Some of the newly found factors could act as maturational checkpoints, to rearrange ribosomal architecture, or otherwise be adaptors for the nuclear export, or even carry more than one function.

A major breakthrough in understanding the nuclear transport was made when Nmd3 was discovered, a protein which carries functions both in the maturation of ribosomes and in its transport (Ho et al., 2000). NMD3-like proteins are present also in prokaryotes, but only in eukaryotic species NMD3 has a C-terminal extension that harbors an NES. The idea that NMD3 shuttles between the nucleus and that cytoplasm was further supported by the finding that leptomycin B inhibits the export of NMD3 (Ho et al., 2000). Additionally, expression of NMD3 mutant lacking the NES results in a nuclear retention of large ribosomal subunits (Gadal et al., 2001). It is also possible that NMD3 has a dual role in ribosomal biogenesis and monitors the assembly by "structural proofreading", where its binding depends on the accuracy of previous steps (Ma et al., 2017). Such a quality control would coordinate the late-maturation with the following export steps, preventing, in general, the export of premature or aberrant ribosomes. NMD3 interaction with the ribosomal subunits is conceivable to be multivalent, where NMD3 might recruit numerous CRM1 molecules. This might represent a general strategy in the transport of large cargoes, which also goes in line with findings from in vitro experiments where multiple NTRs are more efficient in translocation of large cargoes (H. B. Schmidt, 2015).

Another important consideration is an involvement of heterogeneous transport receptors in the transport of large cargos. Indeed, an effective transport of ribosomes requires not only CRM1, but also additional factors, such as exportin 5 (Brownawell and Macara, 2002) and Mex67/Mtr2 heterodimers (Yao et al., 2007). An important aspect of CRM1-mediated 
transport is that it binds ribosomal subunits indirectly, exportin 5 and the Mex67/ Mtr2 dimer, conversely, appear to interact with ribosomal subunits directly (Wild et al., 2010). Certain mutations of MTR2 are also hindering the transport of ribosomes in yeast. The peculiarity of MTR2 is that it functions as a co-factor in mRNA export (Santos-Rosa et al., 1998). These collective findings suggest that distinctive NTRs can play a role in the transport of a cargo, also the number of NTRs, and finally intrinsically properties of the cargo altogether can govern the cargo translocation properties. 


\subsection{Nuclear lamina}

\subsubsection{Lamins are building blocks of the nuclear lamina}

Nuclear processes such as DNA replication, splicing and transcription are temporally and spatially regulated in a strict fashion. The structural integrity of the nuclear content is achieved by more complex structures then a mere lipid bilayer. The inner nuclear membrane (INM), although connected with the outer nuclear membrane (ONM) has a distinct array of approximately 60 integral nuclear proteins, which offer structural support and nuclear plasticity, transduction of mechanical signals (Pajerowski et al., 2007), as well as they regulate cell migration, and finally, cell proliferation (Dechat et al., 2008).

Metazoan structural proteins of the INM constitute a special feature of the nucleus - the nuclear lamina. The Lamina is a $15-20 \mathrm{~nm}$ thick layer of protein meshwork that is directly linked to the inner face of the INM (Dwyer, 1976) Figure 2.5. In contrast to metazoans, lamina is absent in single cell organisms or plants.

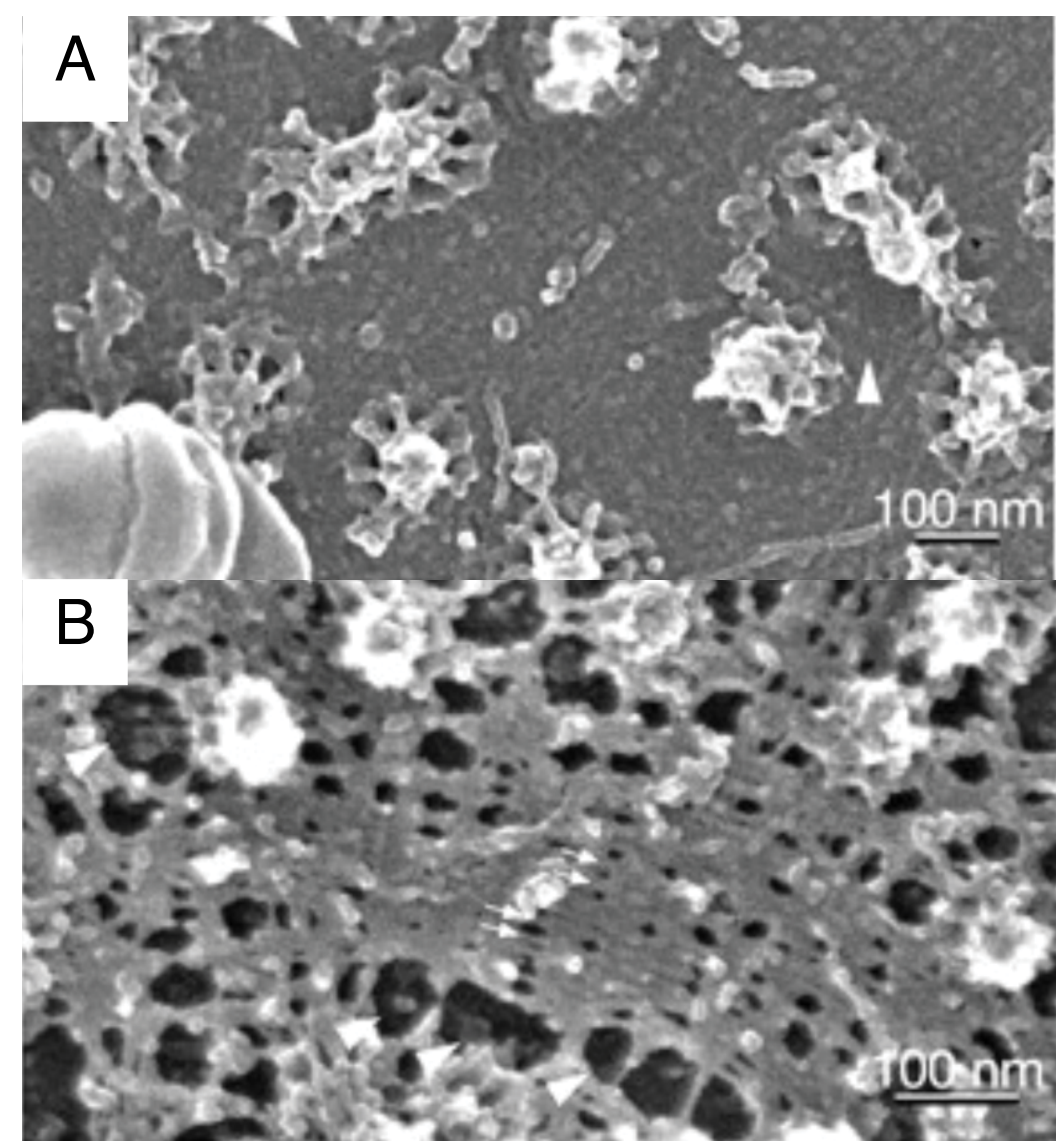

Figure 2.5 Nuclear lamina detected by field-emission scanning electron microcopy. (A) Nuclear face of Xenopus laevis nuclear envelope harboring lamina. (B) Nuclear envelope treated with Triton X100 0,5\% before fixation. Pictures are adapted from Goldberg et al. 2008. 
The nuclear lamina occupies approximately $50-60 \%$ of the inner nuclear membrane surface and constitutes a very dense protein meshwork underlying the nuclear membrane. The lamina protein meshwork is composed of numerous proteins, however, the most abundant structural protein are lamins. Lamins were first identified as 60-80 kDa proteins. Immediately after heir discovery lamins were reported to have architectural relationships to the INM, chromatin, and NPCs (Aaronson and Blobel, 1975; Gerace et al., 1978). Later, lamins were classified as members of the oldest protein family $\mathrm{V}$ of intermediate filaments, from an evolutionary point of view. The term intermediate filaments was proposed based on the fact that the average diameter of assembled lamins is $10-12 \mathrm{~nm}$, which is somewhat in between of actin fibers $(7-10 \mathrm{~nm})$ and microtubules $(25 \mathrm{~nm})$. Two distinct types of lamin were distinguished: A-type and B-type. A-type lamins have a neutral isoelectric point, whereas the type-B lamins are more acidic. Additionally, during mitosis A-type lamins are completely solubilized and not attached to the INM (Gerace and Blobel, 1980). Lamins type-B during mitosis, however, are not soluble but are rather present on distinct types of vesicles (Lourim et al., 1996). Type-B lamins are expressed in almost all cell types, whereas A-type lamins expression is limited to certain development stages. Both types of lamins were reported to create two specific laminas. Different laminas however, although formed by two distinct lamin types, seem to be interdependent (Shimi et al., 2008). Each class of lamins has several members, which are worth discussing more in detail.

\subsubsection{The diversity of lamins}

During the animal evolution there was a clear tendency for an increase in number of lamins. For instance, in Caenorhabditis elegance a single gene of lamin was found, coding for a single Ce-lamin. This protein resembles the functions and properties of both A- and B-type lamins (Riemer et al., 1993). Next, in Drosophila melanogaster two lamin genes were discovered coding for a Dm-lamin (B-type), and a C-lamin (A-type family) (Gruenbaum et al., 1988).

An even greater lamin diversity is found among the vertebrates. Generally, mammals have three lamin genes, which code for lamin A, B1, and B2 proteins. However, the actual number of lamin protein isoforms is far greater due to alternative splicing. Mammalian gene LMNA, for example, codes for lamin A, C, and C2 splice variants, LMNB1 for lamin B1, and finally gene LMNB2 produces lamin B2 and B3 (Peter and Stick, 2012). 
In other vertebrates such as fish, amphibians, reptiles, and birds in addition to the above mentioned three lamin genes there is yet a fourth gene - LMNB3. LMNB3 gene makes the whole lamin family even more teeming, as it codes for three splice variants. Such lamin isoforms as lamin $\mathrm{B} 3 \alpha$, lamin $\mathrm{B} 3 \beta$, and lamin $\mathrm{B} 4$ are transcribed and translated from the LMNB3 gene. Another peculiarity of Lamin B3 is that its expression is almost exclusive to the oocyte and early embryos (Stick and Hausen, 1985).

Undoubtedly, the main role of lamins is their structural support of the nucleus and they represent major blocks of nuclear skeleton. Reversible polymerization is a prerequisite for lamins to carry their functions. Indeed, both lamin type-A and-B form polymers. Although much is still unclear how lamin type-A and -B polymers are assembled and finally differ, general molecular features underlying these processes are somewhat similar. Thus, it is worth discussing molecular features of lamins more in detail.

\subsubsection{Structures and functions of lamin domains}

Despite of the great number of lamin isoforms, all the members of the lamin family share a similar tripartite molecular structure. Lamin molecules are fibrilar in shape and are about $50 \mathrm{~nm}$ in length. The structure of lamins consists of three important elements: an aminoterminal head domain, a centrally located rod domain, and a carboxyl terminal tail domain Figure 2.6.

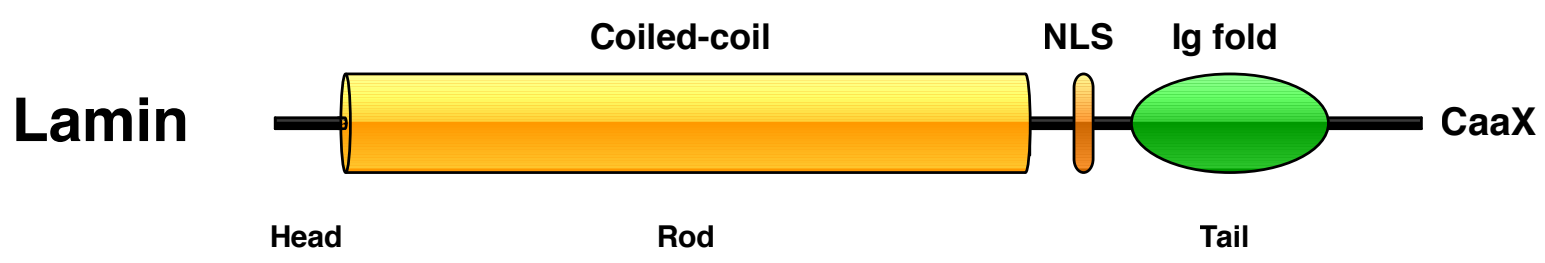

Figure 2.6 General schematic structure of a lamin protein.

The most prominent feature of lamins is the rod domain, which is the central part of all intermediate filament molecules. The rod domain is conceptually similar among all the lamins and the first and the last thirty amino acids are the most conserved regions in the sequence of all lamins. The rod domain is predicted to consist of four $\alpha$-helical regions termed $1 \mathrm{a}, 1 \mathrm{~b}, 2 \mathrm{a}$, and $2 \mathrm{~b}$ plus three short linkers that divide these regions, while at the same time bring flexibility to these regions. Deletions or mutations in the rod domain directly affect the assembly of lamins into higher order polymers. Expression of rod domain deficient lamin B1 in Hela cells causes patchy, lobulated lamina appearance, as 
well as abnormal distribution of other nuclear proteins (Schirmer et al., 2001), indicating a direct role of the rod domain in lamina assembly.

The head domain is an N-terminal disordered stretch preceding the rod domain of lamins. The length of the head domain can vary between 30 amino acids in more primitive metazoans up to approximately 56 amino acids in higher metazoans. The function of the head domain is to facilitate the head-to-tail lamin polymerization. Direct experimental evidences from in vitro studies indicate that recombinantly expressed lamin, lacking the head domain, fails to assemble into the head-to-tail polymers (Isobe et al., 2007). The most important region for the head-to-tail polymers formation was determined to be the last twenty amino acids of the head domain (Isobe et al., 2007). The head domain contains numerous phosphorylation sites and is a target for many kinases and phosphatases, which control the lamin polymerization properties. For instance, Cdk1 phosphorylates the sites conserved among all the lamins (except the C.elegans lamin). Phosphorylation by Cdk1 is crucial for the polymerization/depolymerization of lamins, as shown by in vitro experiments (Eggert et al. 1993). Fish and amphibian lamins contain additional SR/RS dipeptide motifs, which are targeted by serine/arginine protein kinases. This feature was hypothesized to reflect an adaptation of lamins to the biology of oocyte storage in ovaries over a long period of time (Yamaguchi et al., 2013).

As the lamin head domain is involved in head-to-tail polymerization and is a target of numerous protein kinases it is logical to assume that the targeted phosphorylation might keep a fraction of lamins in monomeric form. Likewise, phosphorylation might create local gaps in lamina, facilitating local changes of its physical properties, and ultimately changing lamina-proteome or lamina-chromatin network (Bjerke and Roller, 2006).

The tail domain of lamins is $185-277$ residues long, is mainly disordered, and shows some of the typical qualities of intrinsically disordered proteins such as tendency to aggregate and high proline-glycine content. There are several ubiquitous features that are present in the tail domain of all the lamin family members. First, almost all lamins have a Ras-like Cterminal $\mathrm{CaaX}$ motif, where there is a cysteine, followed by two aliphatic residues and the C-terminal residue. The very C-terminus of lamins is very important as it is a site for protein modifications, including proteolytic cleavage, carboxyl-methylation, while probably the most common modification is isoprenylation (Beck et al., 1990; Chelsky et al., 1987; Farnsworth et al., 1987). Isoprenylation (farnesylation) is a protein modification 
that renders attachment of a hydrophobic moiety to a protein. As all lamins are isoprenylated, it could be logically assumed that this modification is a prerequisite of inner-nuclear membrane localization. Lamins type-A, although have the CaaX motive and are isoprenylated, have also a proteolytic cleavage site. The proteolysis also removes the $\mathrm{CaaX}$ sequence in all type-A lamins after farnesylation, thus ensuring the maturation of lamins type-A. At the same time, although cleaved, lamin A is still incorporated into the lamina (Broers et al., 2002). What's more, when lamins type A are expressed without their very C-terminal 21 amino acids they are still correctly incorporated into the lamina. Lamins type-B, in contrast, are permanently isoprenylated and are stably incorporated into the membrane, even during mitotic nuclear break down (Firmbach-Kraft and Stick, 1993). These data collectively suggest that farnesylation alone is not responsible for targeting lamins to the inner nuclear membrane.

Another very prominent feature found in the lamin tail domain is a so-called IgG-like domain. The IgG-like domain is organized into a compact structure comprised of $\beta$-strands and is about $3.5 \mathrm{~nm}$ in diameter (Dhe-Paganon et al., 2002). The lamin IgG-like domain is suggested to play role in lamin interactions with other proteins and nucleic acids, as well as in lamin polymerization. When recombinantly expressed lamin B3 $\operatorname{IgG}$ is added to $X$. laevis in vitro nuclei assembly, it inhibits further incorporation of lamin B3 into the lamina and blocks nuclear growth (Shumaker et al., 2005).

Lastly, the lamin tail domain contains an NLS, which is an essential element for the nuclear targeting of lamins. The NLS consists of an invariant lysine followed by three basic residues and its relative positioning is variable (Loewinger and Mckeon, 1988).

\subsubsection{Assembly properties of lamins}

Lamins assemble into filaments both in vivo and in vitro, in order to carry their function as nuclear structural elements. Electron microscopy experiments performed on X. laevis oocytes show that in vivo lamins are organized into tetragonal $10 \mathrm{~nm}$ thick filaments (Aebi et al., 1986). Recent studies using cryo-electron tomography suggest that mammalian lamins are organized into the same tetragonal form, however, have only $3.5 \mathrm{~nm}$ in diameter (Turgay et al., 2017) Figure 2.7. 


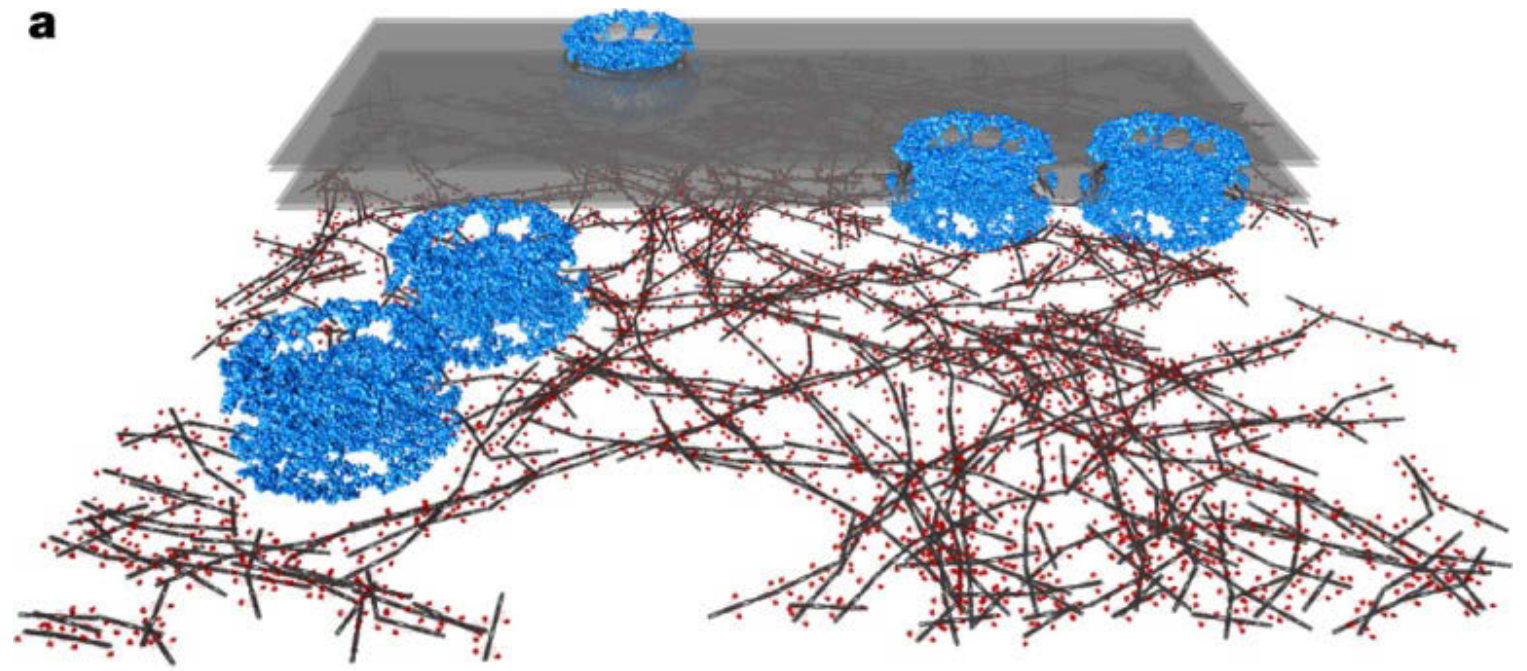

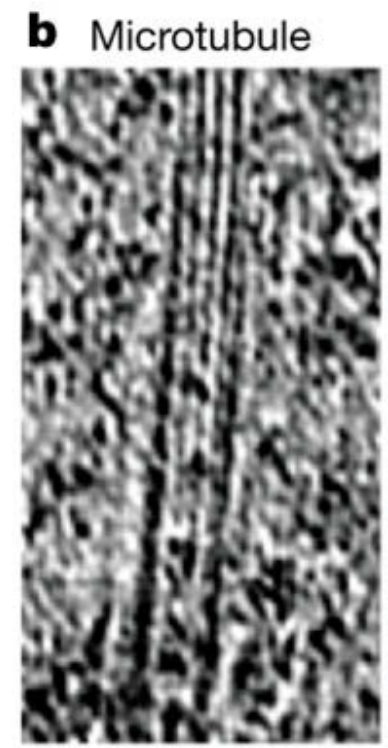

Width: $24 \mathrm{~nm}$

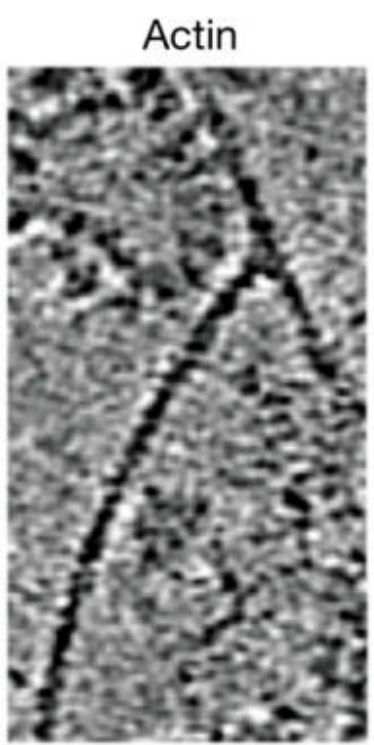

$8 \mathrm{~nm}$

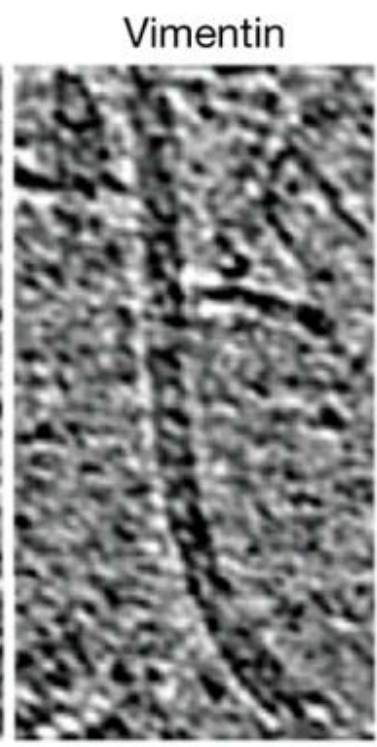

$10 \mathrm{~nm}$

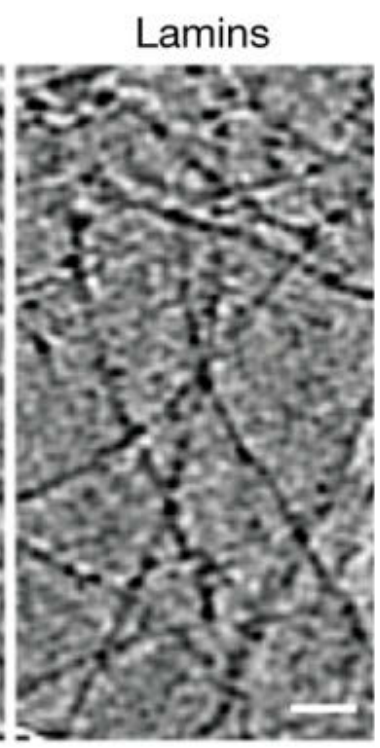

$3.5 \mathrm{~nm}$

Figure 2.7 Nuclear lamina and a comparison of cytoskeleton proteins of mammalian somatic cells. (A) Rod domains are depicted in gray, IgG-like domains are represented by red dots. NPC are shown in blue. (B) Comparison of different types of cytoskeletal elements: microtubule, actin, vimentin, lamins. Pictures are adopted from Turgay et al. 2017.

Resolving the organization and detailed structure of lamina is still a very challenging task, which is attributed to the crowded environment of the lamina and its association with numerous lamin binding proteins and chromatin.

A more detailed overview of lamin organization properties comes from in vitro studies, where lamin polymerization can be induced by changing biochemical conditions of the buffer environment (Karabinos et al., 2003). Lamin recombinant expression in E. coli leads to the formation of inclusion bodies, which have to be dissolved in urea. When urea 
denatured lamin is dialyzed against a basic buffer ( $\mathrm{pH} 8.5)$ lamin dimers are detectable (Aebi et al., 1986; Karabinos et al., 2003), which comprise the very basic building blocks of lamin polymers. The parallel dimers are formed by longitudinal interactions of the rod domains, which create two-stranded coiled-coils. The rod-rod domain interactions resemble a rope-like structure of approximately $50 \mathrm{~nm}$ in length with two distinct globules at one end. The globules represent the $\mathrm{C}$-terminal tail IgG-like domains of lamins. This formation can be induced in vitro by dialyzing lamins against buffers with $\mathrm{pH}$ values around 9 (Aebi et al., 1986; Gieffers and Krohne, 1991).

An unresolved issue in lamin biology is if lamins are able to form heterodimers. Studies on mouse embryonic cell lines suggest that lamins are assembled into homopolymers (Delbarre et al., 2006). More data, obtained using superresolution microscopy, support the notion that different lamin types form distinct independent meshworks (Goldberg et al., 2008). Lamin polymers, observed in vivo, clearly favor formation of homotypical A- and B-type polymers. Such a segregation could be determined by differently controlled transcription, translational, or by selective degradation of heteropolymers prior to their incorporation into the nuclear lamina (Moir et al., 2000).

The next step of lamin polymerization could be reproduced in vitro upon a dialysis of lamin against a more physiological buffer. In a buffer with pH 6 lamins have a strong tendency to associate into head-to-tail polymers with a specific 2-3 nm overlap in their rod domain segment (Gieffers and Krohne, 1991). Later, these head-to-tail lamins start associating laterally into fibrils. The association of the fibrils can have two outcomes, paracrystals or filaments can be formed, depending on the experimental conditions and the type of lamin. Experiments on C. elegans lamin type-B show that lamins yield uniformly polymerized filaments, which are about $10 \mathrm{~nm}$ in diameter, when polymerized in a buffer at $\mathrm{pH}$ 7. These fibrils are indistinguishable from the nuclear lamin fibrils found in vivo (Karabinos et al., 2003). In contrast, when C.elegans lamin B is dialyzed against a buffer with high $\mathrm{CaCl}_{2}$ concentration at $\mathrm{pH}$ 9, long paractrystalline fibers are formed. These paracrystals have a very pronounced dark-light transverse $25 \mathrm{~nm}$ repetitive banding, as detected by electron microscopy.

It must be noted that currently only two types of lamins were assembled into stable filaments. Only lamin B from C. elegans and rat liver lamin type-A were shown to form 10 $\mathrm{nm}$ filaments that resemble lamina in vivo (Karabinos et al., 2003). Filamentous structures 
formed by other lamins are transient, and no $10 \mathrm{~nm}$ filaments could be detected at the steady state (Aebi et al., 1986). For most of lamins lateral association of dimers into paracrystals is strongly favored over the formation of filaments in vitro.

The behavior of lamins in vitro and preferred formation of paracrystals could be explained by the fact that in the physiological environment lamins are an integral part of a supramolecular protein network. Numerous proteins are lamin associated and selectively bind lamins before and after they are incorporated into the lamina. Perhaps, due to the lack of these lamin associated proteins, lamins preferentially form paracrystalls and not intermediate filaments in vitro. Therefore, without all the appropriate lamin binding proteins, only lamin homotypic interactions are captured in vitro, which are never detected in cells in vivo, except when lamins are expressed ectopically or overexpressed. Indeed, when $X$. laevis lamin A is overexpressed in an insect cell Sf9 line paracrystals were observed, which resemble paracrystals assembled in vitro (Klapper et al., 1997). However, when expressed under more physiological conditions lamin A does not form paracrystals and is incorporated into the lamina. This observation was attributed to the expression strength in the insect cell line. Taken together these data show that formation of paracrystals by different lamin isoforms is attenuated or compromised in vivo by cellular factors.

\subsubsection{Lamin functions}

Besides very obvious mechano-structural roles in the nucleus, lamins are also implicated in several other very important functions including remodeling of chromatin, DNA replication, repair and transcription.

Multiple recent studies show that lamins interact with DNA/chromatin. Studies show that lamins interact with mitotic chromosomes (Burke, 1990), lamins have been also identified as peripheral proteins of metaphase chromosomes (Takata et al., 2007). Entire chromosomes seem to be organized into specific nuclear landscapes by lamins (Cremer et al., 2006). It is also suggested that different chromosomes are organized in a specific fashion, where chromosomes poor in genes are closer to lamins and chromosomes with high gene abundance are more distant to lamina (Croft et al., 1999). Notable examples of such chromosomes mapping are the gene-poor chromosomes 13 and 18, normally positioned at the nuclear periphery. In lamin B1 deficient cell lines these chromosomes move away from lamina (Malhas et al., 2007). The positioning of differently active 
chromosomes seems to indicate the involvement of lamins in the regulation of gene transcription. The function of lamins in gene transcription is further supported by evidence that lamin mutations causing lamin depolymerization also lead to impairment of transcription (Spann et al., 1997). Specifically, the activity of RNA polymerase II is inhibited. Overexpression of lamins A or C causes reduction of polymerize II transcription (Kumaran et al., 2002).

Another line of evidences suggests that lamin $\mathrm{A} / \mathrm{C}$ are also involved in the regulation of the cell cycle. This is mediated by an apparent interaction of lamin type-A with a major cell cycle regulator $\mathrm{pRb}$. Hypophosphorylated $\mathrm{pRb}$ is required for cell cycle progression. On the other hand, when $\mathrm{pRb}$ is in its hypophosphorylated state it is tightly associated to lamin A (Mancini et al., 1994). In vitro binding assays show that $\mathrm{pRb}$ is retained in the complex with lamins A/C and LAP2 $\alpha$ (Markiewicz, 2002). Additionally, in lamin A knock-out fibroblasts was observed a dramatic reduction of $\mathrm{pRb}$, pointing towards lamin function in regulation and turnover of pRb (Johnson et al., 2004).

It cannot be excluded, however, that the involvement of lamins in chromosome organization, regulation of the cell cycle, cell proliferation etc. is not achieved via other accessory proteins. For example, studies suggest an additional pathway for lamin/chromosome interactions thorough lamin-binding proteins e. g. LAP2 $\beta$, LAP2 $\alpha$, or heterochromatin protein 1 (Dechat et al., 2004; Ye and Worman, 1996). In general, more experimental evidence is needed to assess direct or indirect regulation of these processes by lamina.

Another interesting function of lamins observed in the field of virology is that replication cycle of cytomegaloviruses and herpes viruses requires assembly of viral capsids in the nucleus, while the maturation of viral particles takes place in the cytoplasm. Thus, the viral capsid envelops at the INM and also fuses with the ONM to gain the access to the cytoplasm. For such an event to occur a virus has to disassemble the lamina. Indeed, experimental data support this notion: lamina disassembly is induced by viral phosphokinases. Human cytomegalovirus phosphokinase pUL97 was shown to phosphorylate lamins in vitro and cause lamin disassembly. Also other viral phosphokinases were shown to function much in the same fashion, namely to phosphorylate and disassemble the nuclear lamina e. g. pUL13, pUL34, BGLF4 (Bjerke and Roller, 2006; Lee et al., 2008). 
To summarize, lamins carry, in addition to their structural functions, carry other important and sometimes essential roles, which including chromosome organization, DNA replication, cell proliferation and differentiation. 


\section{Results}

It is well-established that NTRs facilitate the NPC-passage by mediating interactions with the FG repeats. Nevertheless, the NPC-passage of large (i.e. ribosome-sized) objects is poorly understood. In particular, it was far out of reach to define a minimal set of interactions for conferring NPC-passage competence to a ribosomal subunit. The study of a minimal set of NTRs for a ribosomal transport is complicated by the presence of features that make ribosomal subunits export-incompetent, upon the maturation steps in the cytoplasm. An approach to use pre-mature export competent ribosomes, however, is also limiting, as the NTRs presented on the ribosomes are not easily amenable. In turn, this leads to a limitation, that any further manipulations of NTRs positioning, type, number, cooperative binding are not feasible. Ribosomes from prokaryotic organisms, on the other hand, are similar in size to their eukaryotic counterparts, well characterized, and lack any features for an optimized NPC transport. We argue that such ribosomes could be ideal cargos for in vitro studies of NPC transport and several important conceptual questions can be addressed: what is the minimal set of NTRs for an efficient transport of a ribosomal subunit? Does the distribution of NTRs on the cargo matter? Do cooperative NTRs foster the passage?

A technical challenge that arises is, how to attach an NTR to a prokaryotic ribosomal subunit. We propose that nanobodies raised against ribosomes can serve as an excellent platform to bring NTRs to the ribosomal subunits, provided that they are equipped with an NES signals. Indeed, nanobodies harboring an NES can easily recruit an NTR and, at the same time, offere means to manipulate the number of added nanobodies, the type of NTRs, and NTRs distribution. However, to meet this goal several key objectives have to be met: purification of ribosomes for animal immunization, immobilization onto paramagnetic beads, and in vitro assays, constriction of nanobody libraries, elution optimization of phage displayed nanobodies, as well as expression and characterization of anti-ribosomal nanobodies. In the following chapters we discuss obtained results, introduced technical novelties, as well as possible methodological complications.

The second part of the given study focuses on the production of nanobodies against lamin B3. We describe such important aspects of nanobody production as expression of lamins in a soluble form in E. coli, characterization of anti-lamin nanobodies, application of antilamin nanobodies for microscopy and biochemistry. 


\subsection{Generation of anti-ribosomal nanobodies en route to transport competent ribosomal subunits}

\subsubsection{Purification of 305 and 505 ribosomes from Thermus thermophilus}

Since the discovery of thermophile bacteria, such as Thermus thermophilus, they have become of a significant scientific interest due to the applicability of their thermostable molecules (Oshima and Imahori, 1974). Multiple studies have described a use of thermophile bacteria as a source of proteins for crystallography (Sedelnikova et al., 1987), isolation of useful enzymes e. g. DNA polymerase (Lawyer et al., 1993), and even plasmid preparation and protein expression (Lasa et al., 1992). Thermus thermophilus also served as the very first model organism to provide a ribosomal crystal structure (Ban et al., 2000).

Considering that ribosomes from Thermus thermophilus are well characterized particles and devoid of any features designed for the nuclear-cytoplasmic transport, we reasoned that they might be good candidates to be tested in in vitro transport assays. To meet the objective of obtaining transport competent prokaryotic ribosomes it was absolutely essential to obtain homogeneous $70 \mathrm{~S}$ ribosomal samples, which can be used for raising nanbododies and, eventually, in transport assays. The isolation of ribosomes from thermophilic organisms is a lot more elaborate, in comparison to mesophilic microorganisms, due to numerous proteins, which are tightly bound to the ribosomes. We have purified Tth ribosomes as described by Korostelev et al. 2006 with essential modifications in the procedure Figure 3.1.

Thermus thermophilus culture was harvested at 0.6 OD600 and re-suspended in 1x TNKM buffer supplemented with DTT. We noticed that the Tth cells are practically resistant to the lysozyme action even after $2 \mathrm{~h}$ incubation at $25^{\circ} \mathrm{C}(2 \mathrm{mg}$ of Lysozyme per $\mathrm{ml}$ of cell suspension), that matches the observation made by Oshima and Imahori, 1974. Only cell passages through the French press in presence of DNAse I $(10 \mu \mathrm{g}$ per ml of cell suspension) yielded high protein extraction. Then, to sediment non-soluble cellular fraction we have done fractionated centrifugation, and to enrich and retrieve the $70 \mathrm{~S}$ ribosomal fraction from the lysate we have performed centrifugation into a sucrose cushion. We observed that ultracentrifugation through a $1.6 \mathrm{M}$ sucrose cushion yielded a purer and more homogeneous sample in comparison to $1 \mathrm{M}$ sucrose cushions. A single ultracentrifugation via the sucrose cushion resulted in a formation of a transparent, ribosome-rich, and readily soluble pellet. In view of the reasonably pure pelleted 
ribosomes, we loaded them directly onto a HiTrap Butyl column (GE Gealthcare Life

Science, Germany). The first hydrophobic chromatography step allowed us to get rid of the main contaminants, including ferritin Figure 3.1.
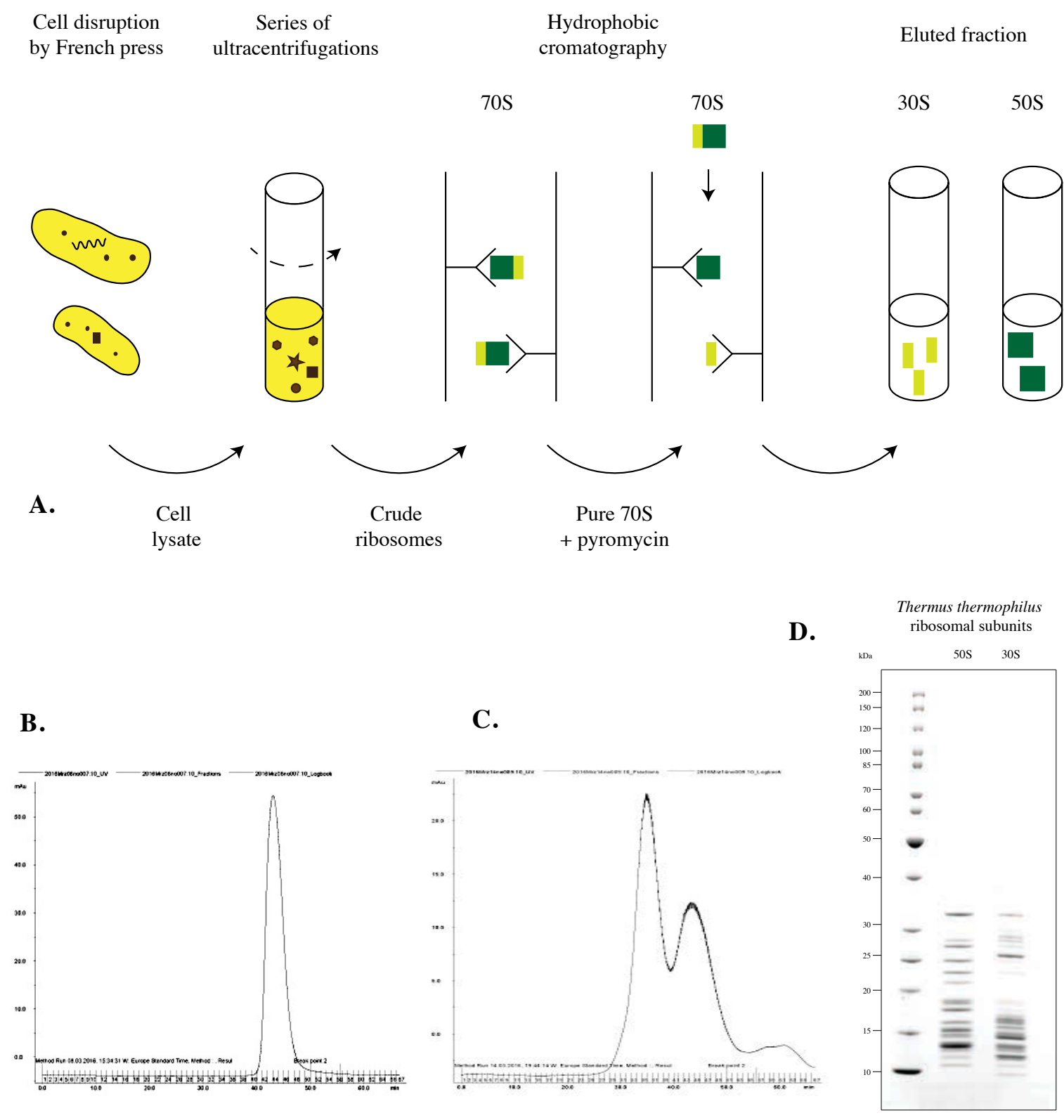

Figure 3.1 Purification of ribosomal subunits from Thermus thermophilus. (A) Illustration of a ribosome purification strategy. Ribosomes pelleted through a sucrose cushion were directly resuspended in 1x TNKM buffer supplemented with $1 \mathrm{M}$ ammonium sulfate and loaded onto a hydrophobic column. Peak fractions (B) were collected and pulled together. To further purify ribosomal subunits and to remove any polypeptide chains from the subunit interface the $70 \mathrm{~S}$ ribosomes were incubated with pyromycin. Incubation of $70 \mathrm{~S}$ ribosomes with pyromycin resulted in $30 \mathrm{~S}$ and $50 \mathrm{~S}$ ribosomal fractions, which were loaded onto the hydrophobic column again. $30 \mathrm{~S}$ and 50S ribosomal fractions were well separated (C) and finally analyzed on an SDS-PAGE gel (D). 
Then, to remove any nascent polypeptide chains and mRNA from the ribosomal interface we treated 70S ribosomes with pyromycin, that causes separation of $30 \mathrm{~S}$ and $50 \mathrm{~S}$ subunits. The $30 \mathrm{~S}$ and $50 \mathrm{~S}$ subunits were loaded onto the HiTrap Butyl column again. The pyromycin treatment together with the second hydrophobic chromatography step allowed us to obtain well separated $30 \mathrm{~S}$ and $50 \mathrm{~S}$ peaks. We have used the ribosomes for all the further experiments e. g. bio-panning, characterization of nanobodies, and FG particles assay.

\subsubsection{Immobilization of Thermus thermophilus ribosomes}

Screening of a nanobody library, to obtain antigen-specific molecules is a multistep process where the first step is an immobilization of a ligand onto a solid support. This step might seem somewhat trivial, however, the way the antigen is immobilized directly influences the selection of nanobodies. Several common methods are available for antigen immobilization for bio-panning. These include antigen immobilization onto plastic surfaces in form of polystyrene tubes (Hust et al., 2002), nitrocellulose (Hawlisch et al., 2001), column matrix (Noppe et al., 2009), and, to the largest extent onto magnetic beads (McConnell et al., 1999). We argued that the direct ligand immobilization by simple adsorption might orient the antigen in such a way as to obscure binding pockets, thus making them inaccessible to displaying phages. Paramagnetic beads, on the other hand, are more advantageous in terms of antigen immobilization. Magnetic beads offer increased surface for binding, as well as thoroughness and ease of washing. Based on these assumptions we decided to immobilize $70 \mathrm{~S}$ ribosomes to paramagnetic beads via a flexible long-arm linker. We have selected an adequately sized $59 \AA$ A long Maleimide-PEG11Biotin linker to immobilize ribosomes (Hansen et al., 1995). Immobilization of ribosomes was done in a two step fashion. First, Maleimide-PEG11-Biotin molecules were coupled to the ribosomes, and then the ribosomes were immobilized onto paramagnetic beads. The structure analysis of $70 \mathrm{~S}$ ribosomes from Thermus thermophilus (PDB accession number 4V4I) (Korostelev et al., 2006b) showed that there are only about nine accessible cysteines on the surface of the ribosome Figure 3.2. 

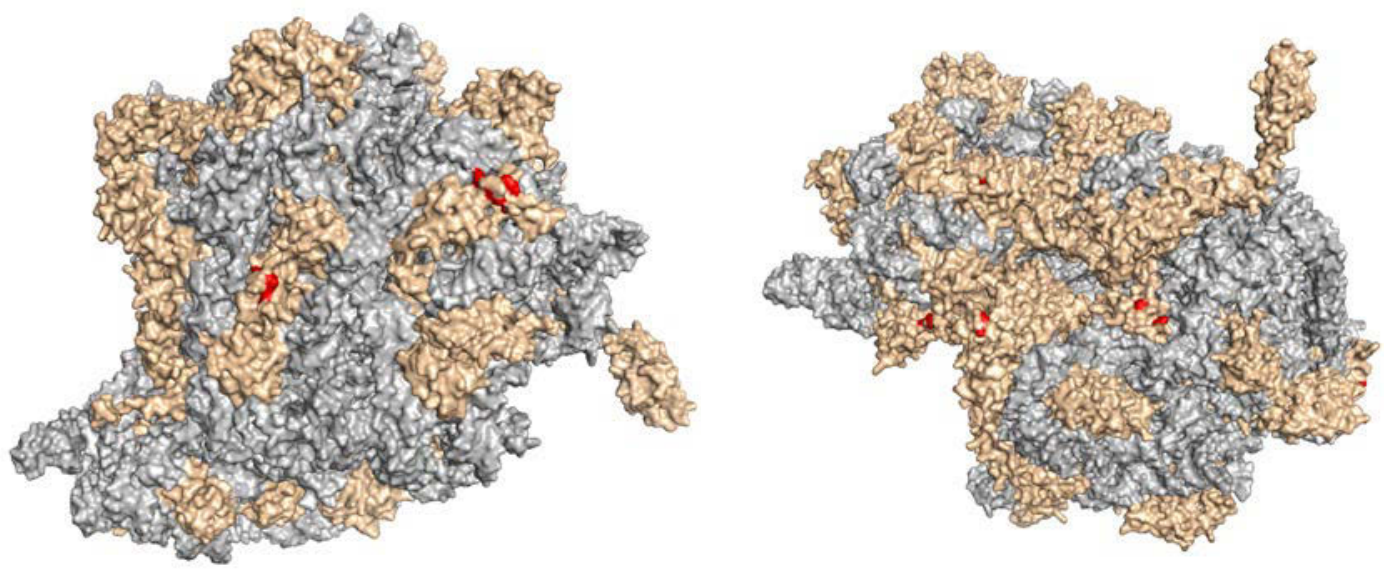

Figure 3.2 Structural analysis of cysteine positioning on the 70S Thermus thermophilus ribosomes. Ribosomal RNA is shown in gray, ribosomal proteins in wheat color, and the cysteines are in red.

We analyzed these cysteines on the surface of the ribosome, to determine if they would be accessible for the maleimide. For that, we coupled Alexa 488-maleimide fluorescent dyes to the ribosomal subunits. This methodology allowed us to assess how many proteins could be potentially bound to the maleimide and also to what extend, as indicated by the intensity of the fluorescent signal Figure 3.3.
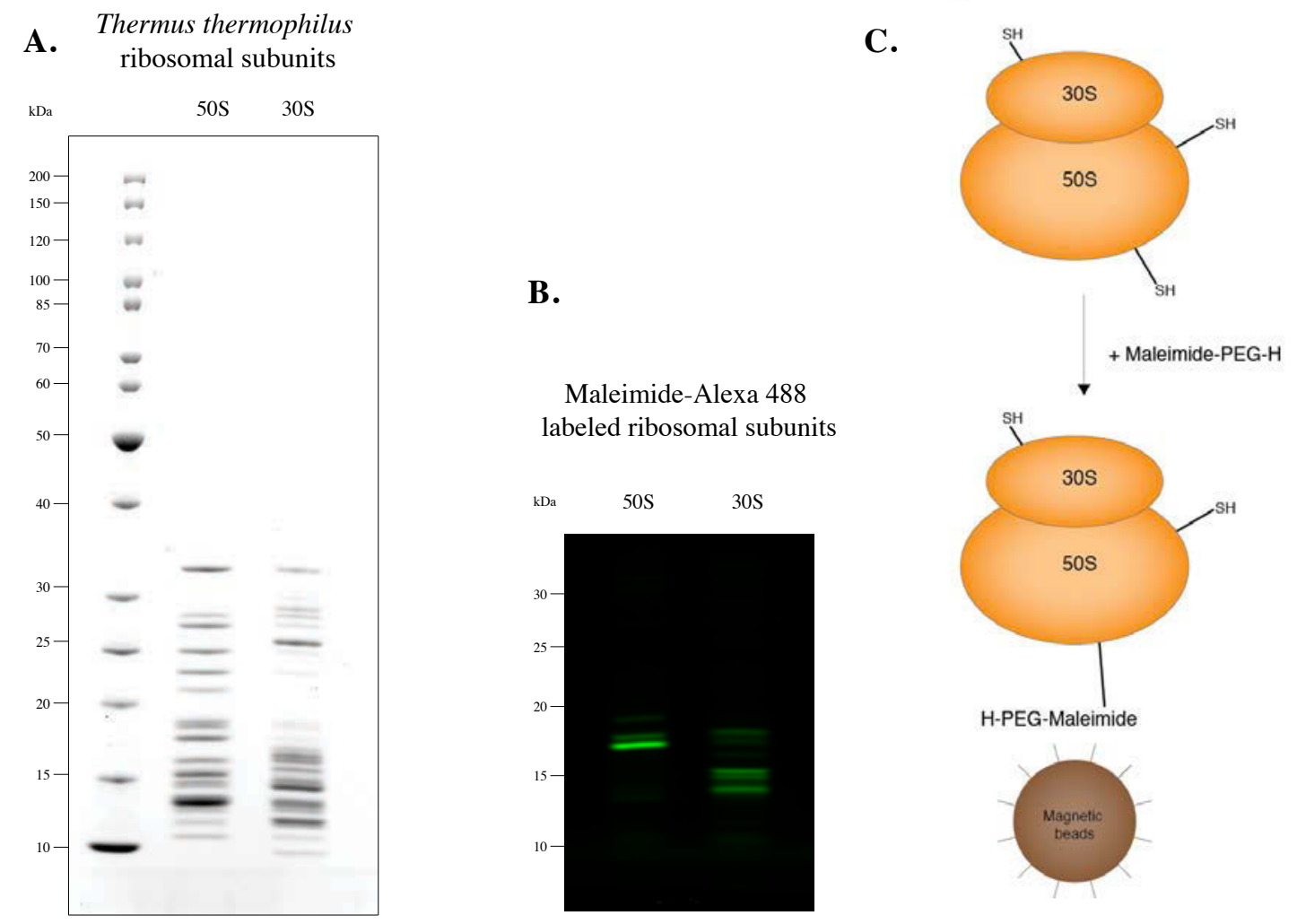

Figure 3.3 Maleimide-Alexa488 fluorescent labeling of Thermus thermophilus ribosomes. (A) 
Purified Thermus thermophilus ribosomal subunits. (B) Maleimide Alexa488 labeled ribosomal subunits. Ribosomal subunits were first reduced with DTT, re-buffered into a potassium buffer $\mathrm{pH}$ 6.8 and the Maleimide-Alexa 488 was added in 1.2 molar excess over the concentration of the ribosomal subunits. Note, that the overall number of proteins reacted with the maleimide dye closely corresponds to the overall number of the surface cysteines. (C) A scheme illustrating a general strategy of immobilization of ribosomes onto paramagnetic beads. The ribosomes were first reduced with DTT and then re-buffered into a potassium buffer $\mathrm{pH} 6.8$ and coupled to MaleimidePEG-H. Biotinylated ribosomes were then bound to streptavidin magnetic beads.

The immobilization of ligands to streptavidin consistently yielded low background, therefore we routinely used Maleimide-PEG-H to immobilize bulky molecules that otherwise cannot be bound to the paramagnetic bead matrix.

\subsubsection{Generation of SUMO protease cleavable phages}

A critical prerequisite for any bio-panning procedure is a successful recovery of positive phages, which are bound to an immobilized target. Ideally, this process should be specific e. g. allowing the elution of phages based only on interactions of a displayed protein with a ligand. This process should also not cayse any phage modifications that might compromise their infectivity. And lastly, it should be preferably time efficient, so that it does not add up a lot to the overall time of the bio-panning procedure.

A standard way to elute phage particles is a pH elution. This method is based on a simple disruption of interactions between the displayed protein and the immobilized target and/or denaturation of these proteins. The $\mathrm{pH}$ elution requires a harsh treatment of phage particles either with triethylamine ( $\mathrm{pH} \sim 12$ ) (Martineau, 2010) or with glycine/ $\mathrm{HCl}$ ( $\mathrm{pH} \sim 2$ ) (Steiner et al., 2008). Although the $\mathrm{pH}$ elution was used in numerous studies, it has several serious limitations. First of all, phages that display nanobodies with the highest affinities might be very difficult to be dissociated without destroying the phage itself and loosing phage infectivity. At the same time, the harsh $\mathrm{pH}$ elution elutes phages, which were retained for a wrong reasons e. g. unspecific binding.

An alternative method is protein cleavage by trypsin (Breitling et al., 1991). This method relies on the action of trypsin, that cleaves proteins at the carboxyl side of the amino acids lysine or arginine. Thus, when these amino acids are introduced between the displayed protein and the pIII, trypsin is able to cleave the virions. A drawback of the given methodology is that trypsin treatment is not specific and can lead to a partial digestion of phage proteins that in turn might cause loss of phage infectivity. Also, trypsin digestion is inefficient and requires high amounts of the enzyme and long incubation times. 
The $\mathrm{pH}$ elution method does not discriminate between a background phage binding and a specific phage binding, leading to a further propagation of unspecific phages. Therefore, we speculate that a development of a specific proteolytic cleavage of phage particles from the displayed protein could allow a mild cleavage at physiological conditions and maintain high phage infectivity Figure 3.4.

\section{Site specific protease elution of M13 phages}

Cleavage of specifically bound phages

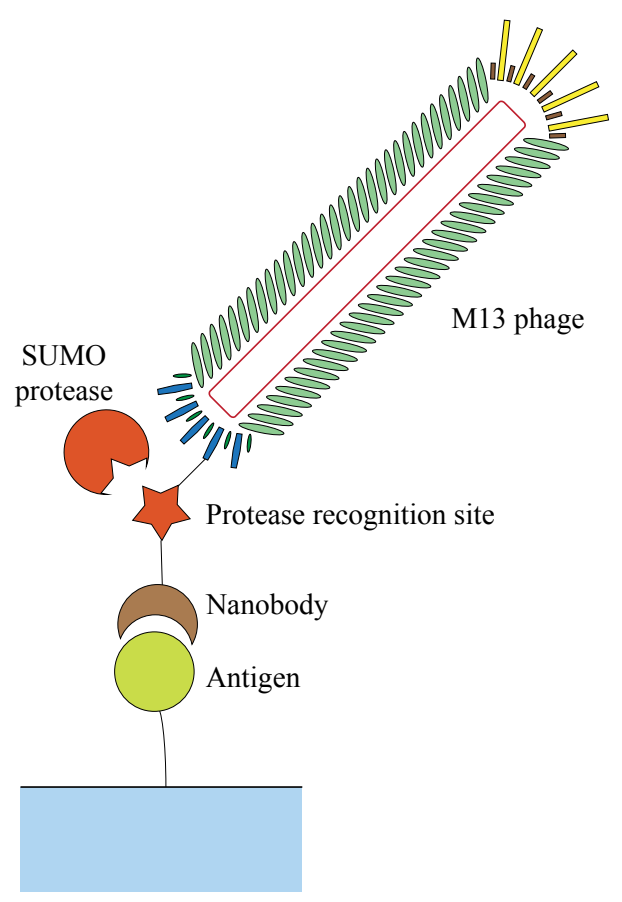

Free eluted phages

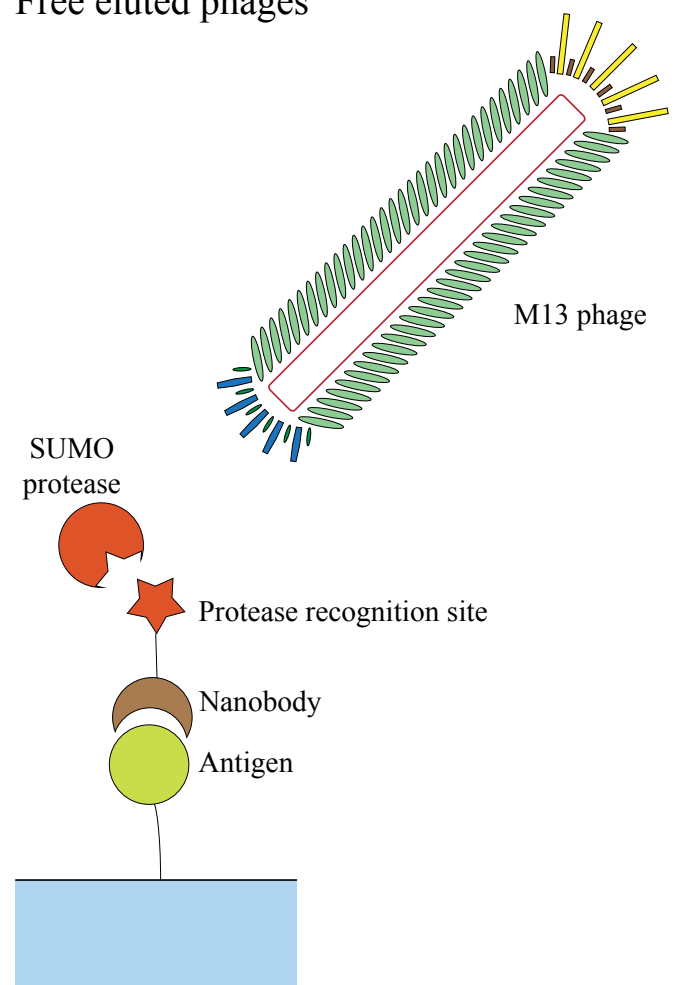

Figure 3.4 Site specific protease elution of M13 phages. (A) Protease cleavable phages are bound to a specific antigen via a displayed protein. (B) A protease cleaves the protease recognition site, thus freeing the phage particle. The major coat protein pVIII is depicted in green, pVII in yellow, pIII in blue.

First, we tested if an introduction of a cleavable module between the displayed protein and the pIII would result in viable phages. For that we introduced a TEV cleavage site, as well as an enterokinase and a SUMO cleavage site (Frey and Görlich, 2014) into a phagemid of the bacteriophage M13 Figure 3.5.

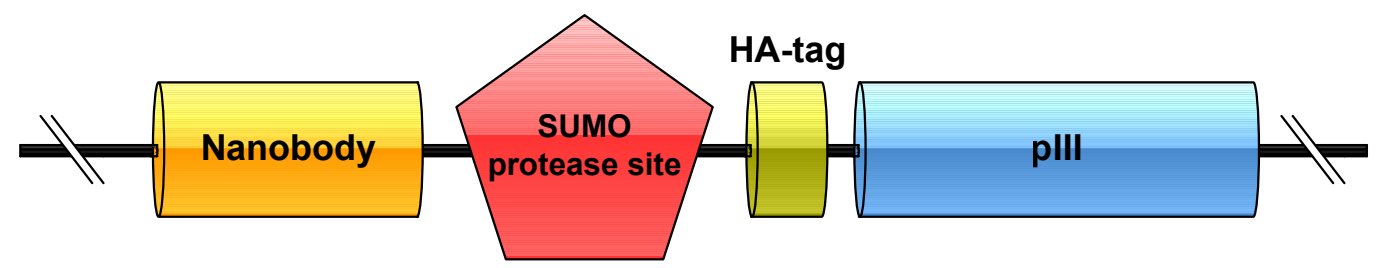

Figure 3.5 An open reading frame of a phagemid M13 with engineered protease cleavage 
sites.

Both the TEV and the enterokinase are common tools in molecular biology. TEV and enterokinase kinases recognize short peptide sequences; on the contrary, the SUMO protease recognizes a folded $10 \mathrm{kDa}$ cleavable domain. First, we tested if the largest SUMO tag would be correctly folded and exposed on virions. We cultured protease cleavable phages and harvested them as described in the "Harvesting and purification of bacteriophages" section. Then, by means of western blotting we confirmed that the SUMO cleavable domain is displayed on the phages together with a nanobody Figure 3.6.

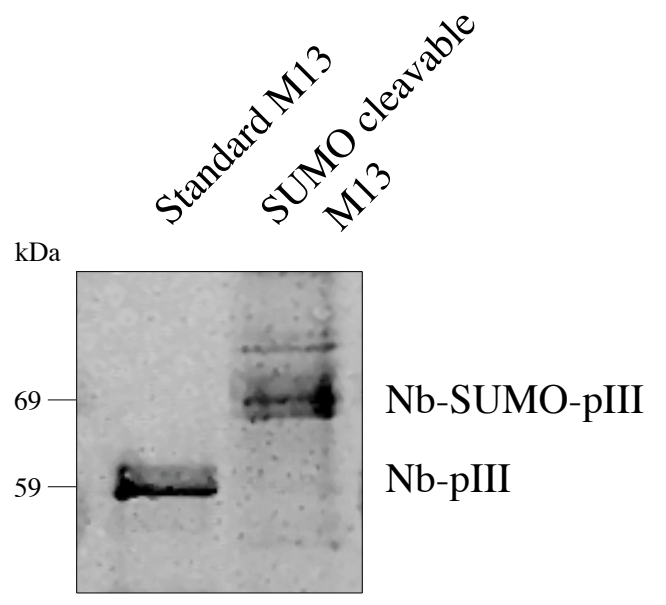

Figure 3.6 Western blot analysis of SUMO protease cleavable phages. Virions carrying the SUMO cleavable site were separated by SDS-PAGE followed by Western Blot. Phages displaying nanobody or nanobody-SUMO fusions were detected by anti-HA tag antibodies.

Based on these positive data we wanted to test if protease cleavable phages can be successfully used for bio-panning of nanobodies, as well as to evaluate the most efficiently cleaved domains. We set up an experiment where we bound equal amounts of phages with different cleavage modules to an immobilized antigen. The phages were stringently washed to ensure that only specifically bound phages are left bound to the antigen. Phages were eluted with TEV, enterokinase, and SUMO proteases and the amounts of virions were estimated by phage tittering as described in the "Phage particles quantification section" Figure 3.7 . 


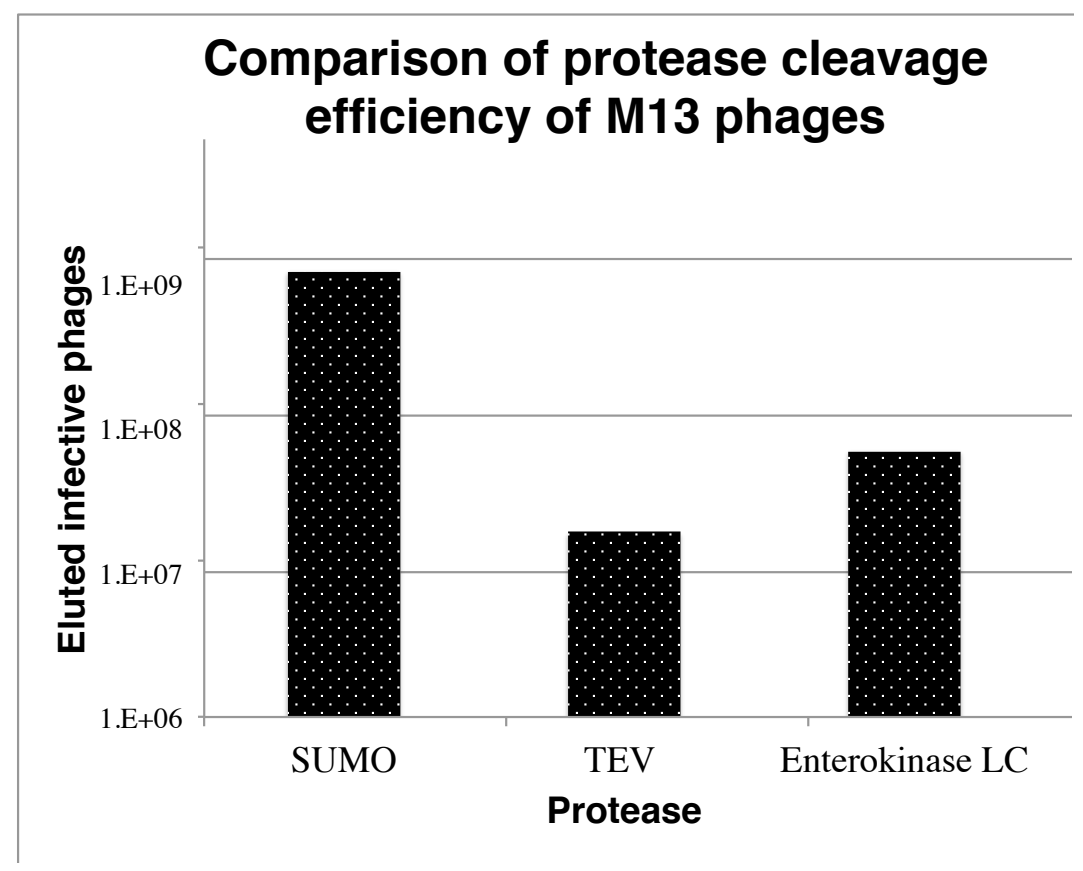

Figure 3.7. Characterization of engineered protease cleavable phages. Phages were eluted with SUMO protease, TEV protease, and enterokinase (LC). The elution time of SUMO domain was 5 minutes at $37^{\circ} \mathrm{C}$, of the TEV protease and enterokinase 3 hours. The samples were normalized against the negative control, where there was no protease added.

Eluted phages were all viable and exhibited high infectivity. Also, in the case of SUMO cleavage, the number of eluted phages closely matches the number of ligand molecules immobilized onto the support. As seen in the Figure 3.7 the SUMO protease showed the highest efficiency of virions cleavage, together with the shortest time required for the reaction. The SUMO domain served as an excellent tool for a fast and specific elution, therefore, we used the SUMO-protease cleavable phages to display nanobodies in all the following experiments.

\subsubsection{Selection of anti-ribosomal nanobodies and their characterization}

Phage display serves as a great technological tool to rapidly screen large libraries of proteins (Smith, 1985). To select nanobodies against 70S Tth ribosomes, we have used magnetic beads immobilized ribosomes for recurrent bio-panning rounds. In total, we have performed four rounds of affinity selection, which, on average, is an adequate number of cycles and should not lead to undesirable over-selection. It's worth mentioning that the concentration of immobilized ribosomes in the very first round of panning was $0.5 \mathrm{nM}$ and it was reduced to $0.25 \mathrm{nM}$ in the last round. Also, to further boost the selection pressure, in every consecutive round of panning the number of washes was increased, reaching 14 . 
After the fourth round of panning we have obtained $\phi$ circa $6 \times 10^{3}$-fold phage enrichment over the background binding, which is an indication that the selected phages were ready for sequencing. Before that, the selected nanobodies had to be re-cloned into a characterization vector. To reduce cumbersome manipulations associated with one by one cloning of nanobodies we have cloned the nanobody library in bulk. Such a cloning reduces hands-on time and prepares all the nanobodies for any further characterization e. $\mathrm{g}$. antigen pull-down or fluorescent microscopy. A common characterization vector used in this work is depicted in Figure 3.8.

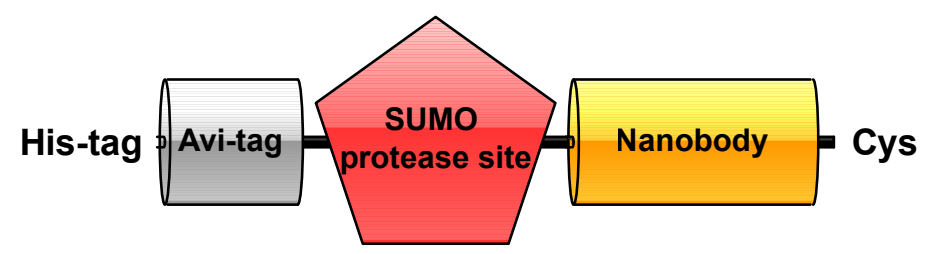

Figure 3.8 Open reading frame of the nanobody characterization vector

The Avi-tag allowed a rapid immobilization of the bait nanobody onto streptavidin magnetic beads and the SUMO-cleavable module offers an easy way to elute a captured prey. In total, 96 individual E. coli colonies were picked for sequencing. The sequences were aligned and analyzed according to nanobodies CDRIII loops. We have used Ugene software to align nanobody sequences and build phylogenetic trees Figure 3.9 (Okonechnikov et al., 2012). The panning against 70S ribosomes resulted in several distinct nanobody classes from which we picked at least a single representative nanobody for characterization. In total, we have analyzed 10 anti-ribosome nanobodies from the forth panning and 4 nanobodies from the third panning. All 14 nanobodies were expressed in $E$. coli and purified on $\mathrm{Ni}^{2+}$ matrix. To characterize these selected nanobodies and confirm their specificity towards the antigen we have done a series of immunoprecipitation experiments.

A.

FWIII

CDRIII

FWIV

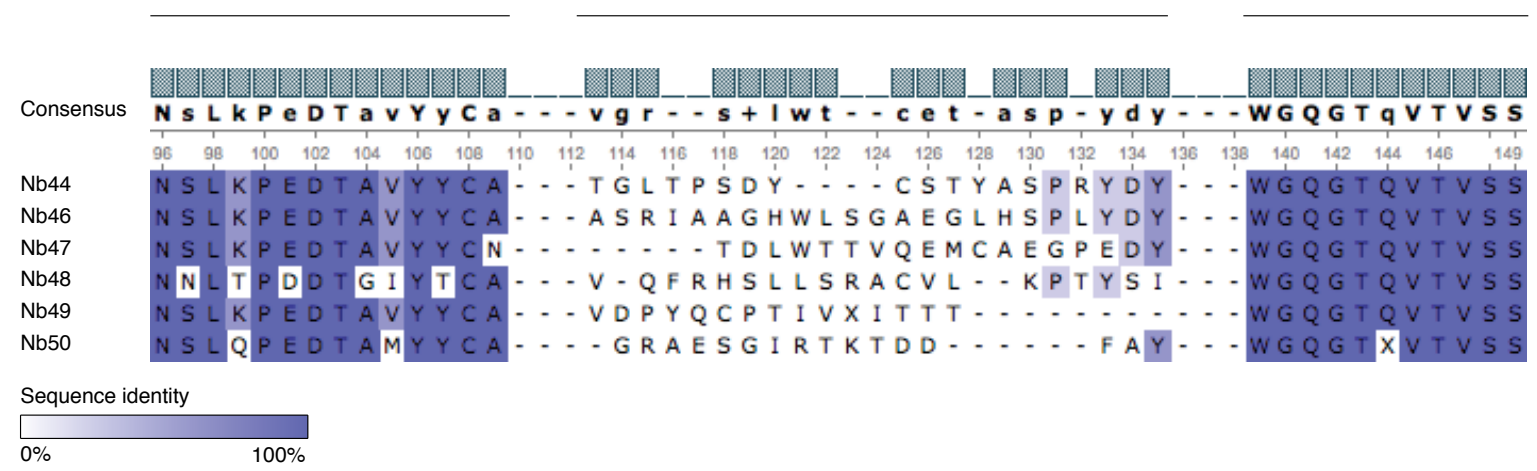


Figure 3.9 Sequence and phylogenetic analysis of nanobodies against $70 \mathrm{~S}$ ribosome from Thermus thermophilus. (A) Sequence were aligned using UGENE (Muscle algorithm) according to their CDRIII loops, note the sequence identity bar. CDRIII loop length varies greatly among the selected nanobodies.

(B) Circular phylogenetic tree of all 93 sequenced nanobodies. Orthogonal nanobodies with unique binding sites are specified.

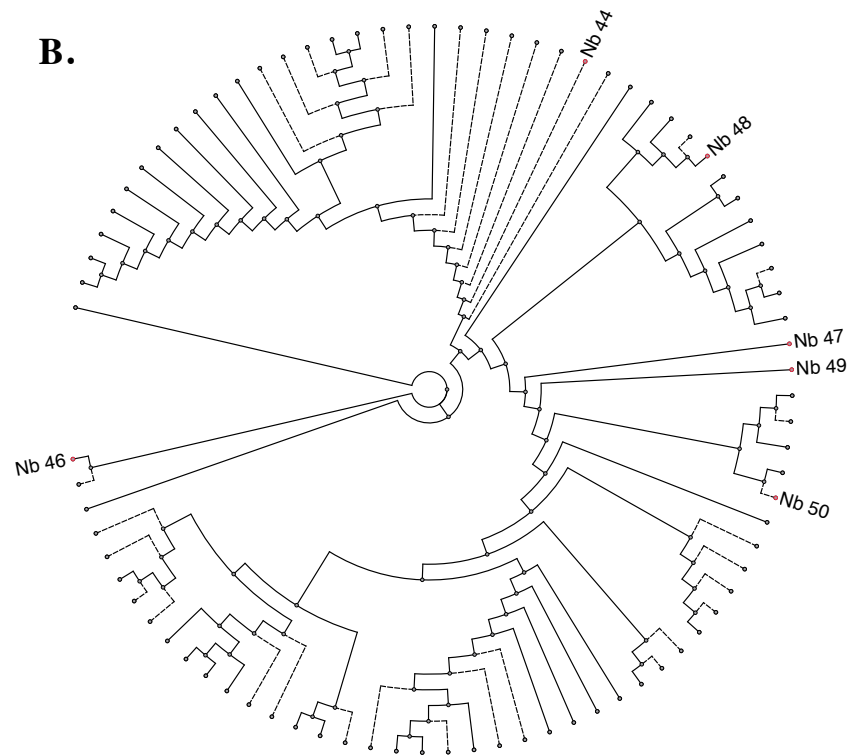

A common method to immobilize nanobodies for affinity chromatography is based on the N-hydroxysulfosuccinimide (NHS) chemistry (Rothbauer et al., 2007). We, however, take advantage of the Avi-tag and co-express, together with nanobodies, the BirA enzyme that biotinylates the Avi-tag in vivo (Schatz, 1993). In order to confirm the specificity of selected nanobodies they were immobilized and directly incubated with Tth cells crude lysate, which was prepared by French press cell disruption, followed by a single ultracentrifugation. In order to establish the binders of $30 \mathrm{~S}$ and $50 \mathrm{~S}$ subunits we treated the lysate with pyromycin. Pyromycin blocks the association of ribosomal subunits allowing affinity capture only of the target subunits by nanobodies. Native isolation of the ribosomal subunits confirmed that two nanobodies are positive binders of the $30 \mathrm{~S}$ subunit and nine nanobodies bind the 50S subunit Figure 3.10. 


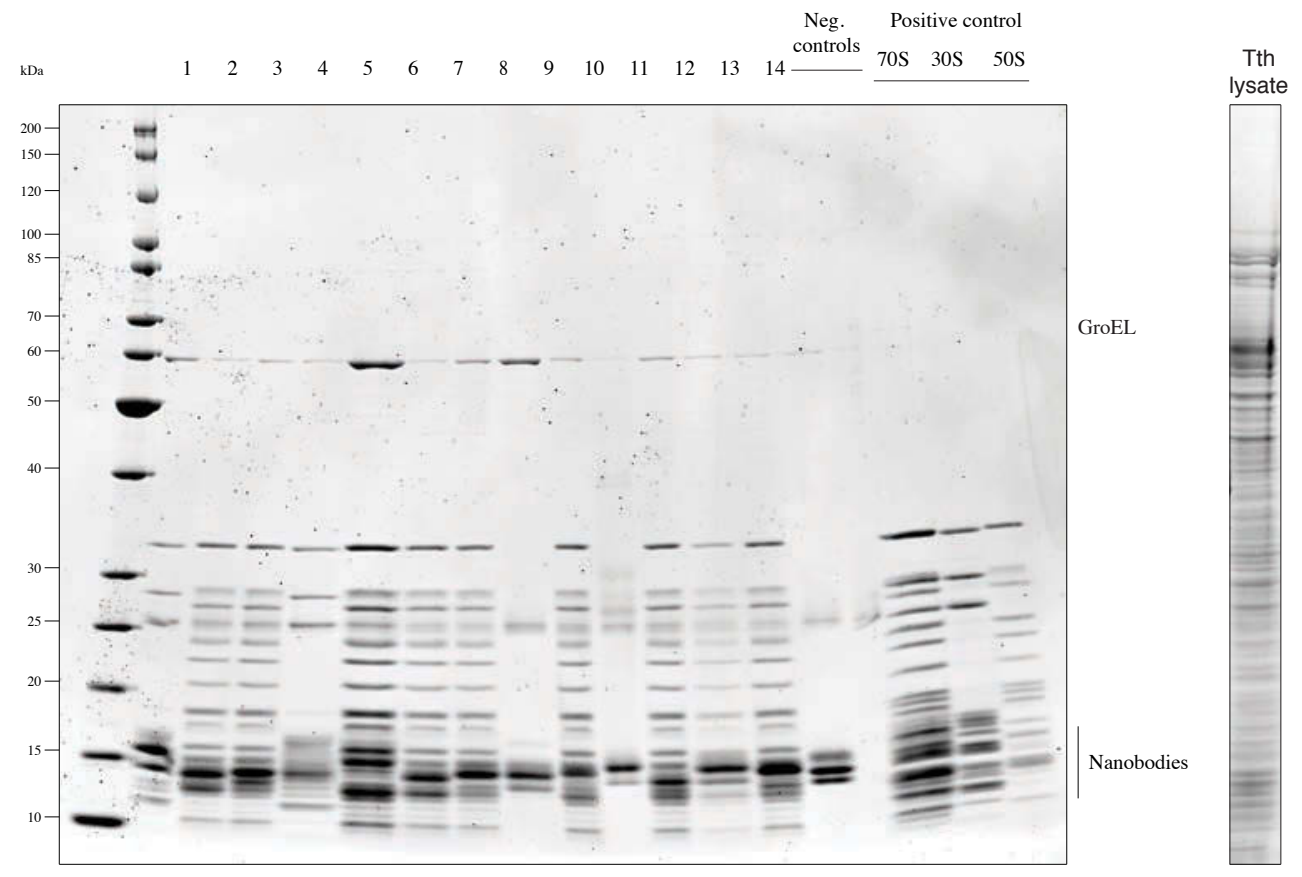

Figure 3.10 Native isolation of ribosomes from Tth lysate. Isolation of native ribosomes from the Tth lysate incubated with pyromycin. Nanobodies 44 (lane 1) and 46 (lane 4) are binders of the $30 \mathrm{~S}$ subunit, while remaining 9 nanobodies specifically target the 50S subunit. Note, 70S, 50S, and $30 \mathrm{~S}$ ribosomes purified by hydrophobic chromatography serve as positive controls. The ribosomal subunits were isolated from crude lysate (lane on the right hand side). Mass spectrometry analysis shows co-isolation of Tth GroEL.

Selected nanobodies allow the enrichment the $70 \mathrm{~S}$ ribosomes and in the presence of pyromycin they are able to specifically bind only the target subunits. Affinity chromatography confirmed specificity of selected nanobodies against the Tth ribosomes.

\subsubsection{Identification of binding partners of nanobodies by cross-linking}

The in vitro transport assay of ribosomal subunits required determination of binding sites of nanobodies. Due to the nature of the bio-panning against full ribosomes and numerous selected nanobodies the process of antigen identification has to be efficient and well optimized. Different approaches are available for characterization of protein complexes. These include, but are not restricted to, crystallization of protein complexes (Chug et al., 2014), cryo-electron microscopy structural studies (von Appen et al., 2015), generation of truncated versions of proteins, or cross-linking coupled to mass spectrometry (Combe et al., 2015). Strategies that involve crystallography or cryo-EM structural analysis could hardly be considered as high-throughput methods for epitope-mapping. We, therefore, 
reasoned that mass spectrometry analysis might be the most convenient epitope mapping strategy to determine binding partners of eleven anti-ribosomal nanobodies.

Numerous cross-linkers are available for conjugation of different protein functional groups, the -SH group is one of most suitable functional groups for cross-linking. Hence, we equipped nanobodies with an external $\mathrm{N}$-terminal cysteine that serves as a primary site for cross-linking (Pleiner et al., 2015). For all the nanobody-ribosome cross-links we have used bifunctional cross-linkers, which bind to cysteines on one hand, and on the other hand to lysine $\varepsilon$-amino groups. The spacer arm of these cross-linkers was either $7.4 \AA$ or $9.2 \AA$, that allowed cross-linking only of proximal groups and provided more detailed information about the complex.

Another important consideration for a successful mass spectrometry analysis is an enrichment of cross-links. There are several protein tags that can be used in affinity purification of complexes for mass spectrometry. For our purpose, we fused a His-tag to nanobodies, which serves as a simple generic affinity handle Figure 3.11. The strategy employing the His-tag nanobody fusions offers an easy sample preparation and reduces hands-on time for the isolation of cross-links. Yet, ribosomes are very large rRNA/protein complexes, which consist of over 50 ribosomal proteins. To reduce the complexity of the samples and at the same time to enrich the cross-links, all non-crossed proteins, ideally, should be removed from the samples. For that, the His-tagged nanobodies were crosslinked to ribosomal proteins and then incubated with SDS containing buffer. The SDS treatment results in a complete denaturation of all the proteins, at the same time leaving the His-tag fully intact and able to bind to $\mathrm{Ni}^{2+}$-matrix. The cross-links were bound to the $\mathrm{Ni}^{2+}$ matrix and eluted with imidazole, this also led to the concentration of the samples, which were directly loaded onto an SDS-PAGE gel for analysis.

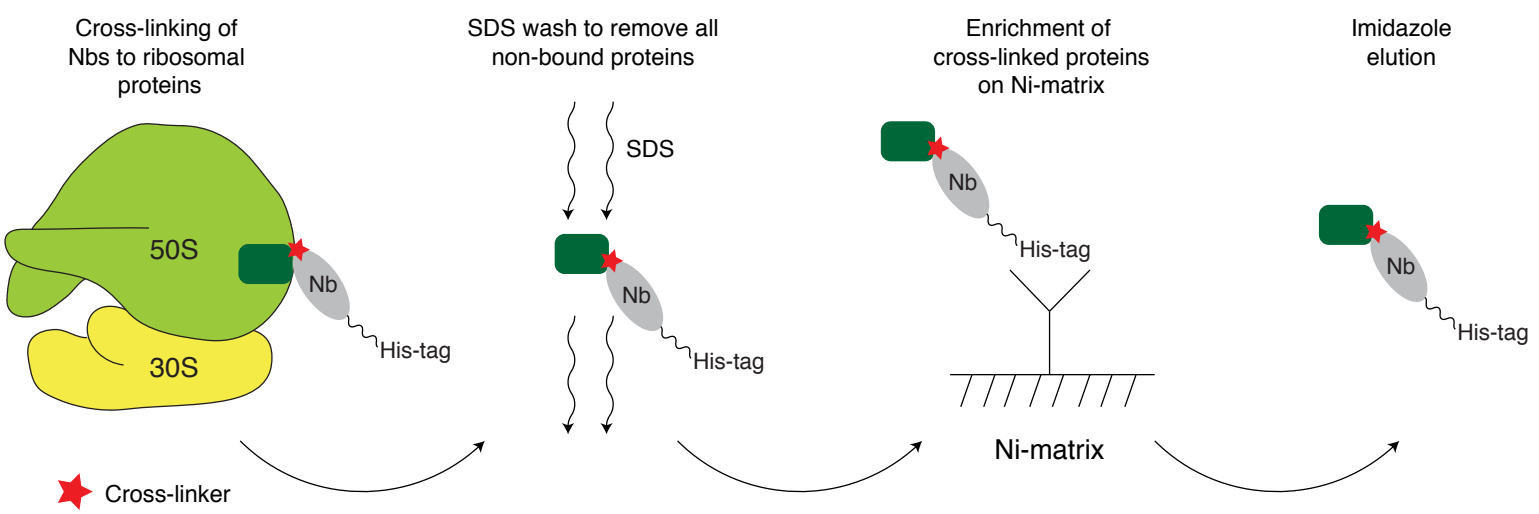

Figure 3.11 Schematic illustration of nanobody-ribosomal protein enrichment strategy. Cross- 
linking was performed in solution. Sulfo-EMCS cross-linkers were added in 2-fold molar excess over the concentration of the nanobody. An SDS containing buffer was added upon $1 \mathrm{~h}$ incubation and the cross-links were directly immobilized onto $\mathrm{Ni}^{2+}$ matrix via the His-tag. Imidazole eluted samples were directly loaded onto an SDS-PAGE gel for analysis.

SDS-PAGE analysis of cross-links shows the efficiency of protein denaturation strategy Figure 3.12. There are virtually no other proteins except the tagged nanobodies and nanobody-ribosomal protein cross-links. Typically, after cross-linking the proteins are hydrolyzed using endopeptidases, which yield a mixture of peptides and covalently linked di-peptides, that can be separated by LC-MS/MS, in order to obtain sequence information. When analyzing the SDS-PAGE gel one could also notice additional bands running between 22 and $28 \mathrm{kDa}$. Mass spectrometry analysis shows that these bands correspond to the inter-molecular nanobody cross-links. Bands corresponding to the non-cross linked nanobodies, nanobody-ribosomal protein cross-links, and intra-nanobody cross-links were excised and sent for mass spectrometry analysis for protein identification Figure 3.12. As well as whole lane digestion was done to evaluate possible less prominent cross-links. All the mass spectrometry work was performed in Prof. Urlaub's laboratory by Monika Raabe and Annika Kuehn.

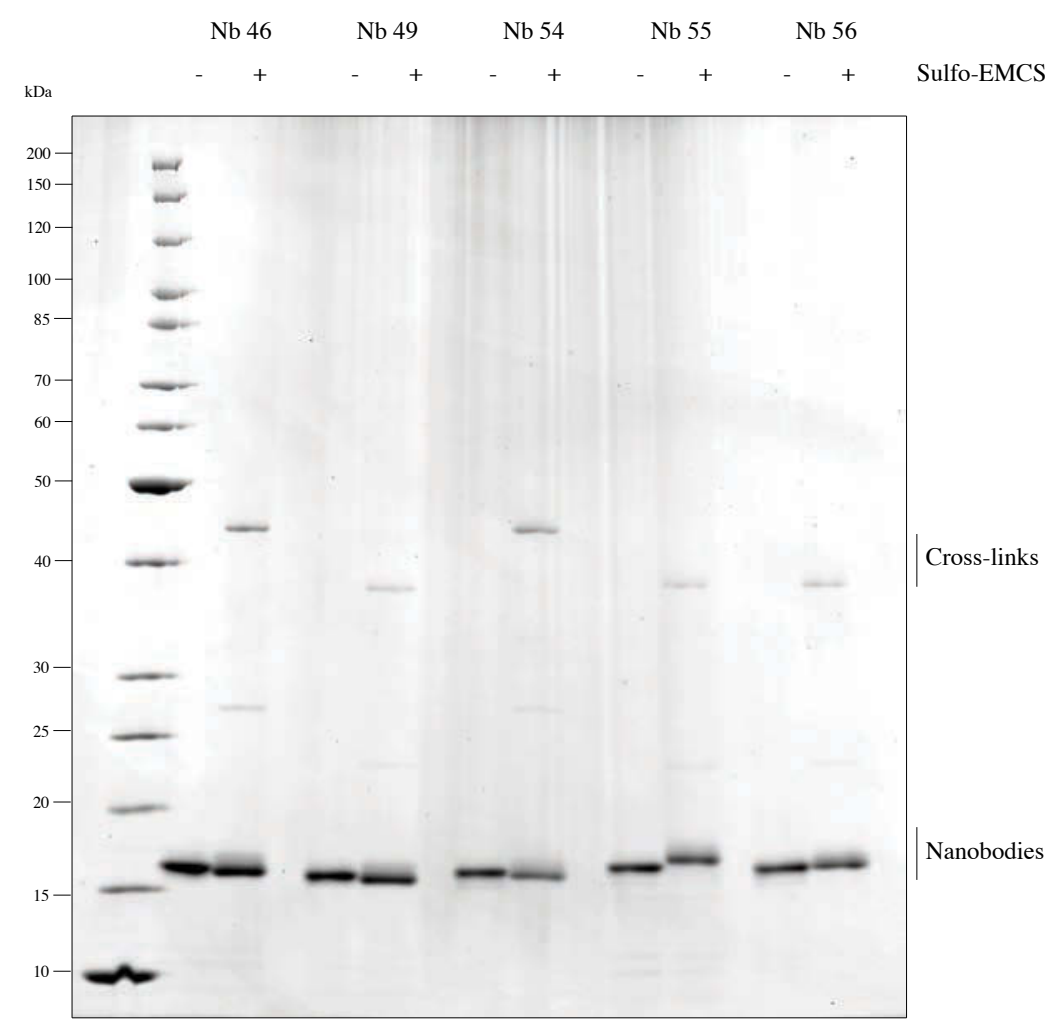

Figure 3.12 Exemplary SDS-PAGE electrophoretic analysis of nanobodies cross-linked to ribosomal proteins. Nanobody-ribosomal protein cross-links migrate at a higher molecular weight than the nanobodies alone. The cross-links were obtained with a $7.4 \AA$ arm bi-functional cross- 
linker. A His-tag was used to selectively enrich the nanbodies.

All analyzed nanobodies were found to be cross-linked to single ribosomal proteins. Mass spectrometry analysis of nanobody cross-links confirmed five unique orthogonal nanobodies against ribosomal proteins Figure 3.13.

\begin{tabular}{|c|c|c|}
\hline $\mathrm{Nb}$ & Target protein & Color code \\
\hline 44 & $\mathrm{~S} 9$ & Red \\
\hline 46 & L1 & Green \\
\hline 47 & S3 & Bleu \\
\hline 48 & L7/L12 & Yellow \\
\hline 49 & L4 & Forest green \\
\hline
\end{tabular}

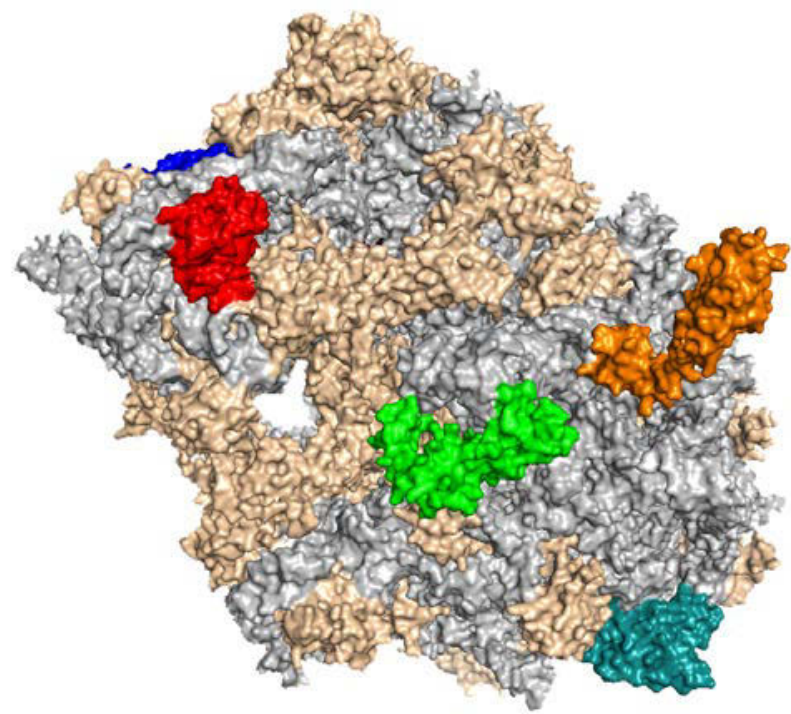

Figure 3.13 Structure of $70 \mathrm{~S}$ ribosomes from Tth with highlighted nanobodies binding proteins. Refer to the table on the right for the legend. Note, that ribosomal protein L7/12 is missing in the structures of the ribosomes, instead the stalk protein L11 (yellow) is highlighted, to which six copies of L7/12 bind. rRNA is depicted in gray, ribosomal proteins are in pale yellow.

The following ribosomal proteins were identified to be targeted by the nanobodies: S9, S3, L1, L4, and L7/12. S3 and S9 are small subunit ribosomal proteins, whereas L1, L4, and L7/12 belong to the large subunit. The ribosomal protein L7/12 is present in 6 copies per Tth ribosome (Diaconu et al., 2005). L7/12 has a flexible hinge region, thus, it was never co-crystallized together with the ribosomes (Diaconu et al., 2005). Probably, due to the fact that it is present in multiple copies per ribosomal subunit the bio-panning to L7/12 was more favored in comparison to other proteins presented in a single copy per ribosome.

We show that the epitope mapping by covalent cross-linking and mass spectrometry analysis is a suitable tool for identifying the interaction partners of nanobodies. It becomes specifically important, when antigen mapping is required for an array of nanobodies and the antigen is large multimeric protein. 


\subsubsection{Identification of rRNA-binding nanobody by CRAC}

During the identification of ribosomal target proteins of nanobodies, we came to the conclusion that it was not possible to obtain cross-links to Nb50 using protein-protein cross-linkers. First, we assumed that the cross-linker spacer arm of $7.4 \AA$ might be too short and thus, it could not access the reactive groups on the ribosome. To test this assumption we have used several maleimide-NHS cross-linkers with spacer arms of 14, 24, 38, and $56 \AA$ respectively (Chen et al., 2010). To our surprise, there were no detectable cross-links in the presence of any of the above mentioned cross-links, especially given that $56 \AA$ is a considerably long spacer arm, which is approximately 5-6 nm in length. There are two possible explanations for this, first there might have been no reactive groups towards NHS in the vicinity of the Nb50 antigen. Another interpretation of this experiment could be that the Nb50 is an RNA binder. To our knowledge, there is no nanobody described, so far, that binds nucleic acids. In this case, given that the rRNA constitutes approximately $65 \%$ of the ribosome, potentially, there are good chances that such rRNA binders could have been selected.

To test the hypothesis of rRNA binding nanobody we next attampted to cross-link Nb50 to ribosomal rRNA (this work was done in the collaboration with Prof. Bohnsack laboratory, where they were provided with all the necessary proteins for the analysis). Protein-RNA cross-linking relies on covalent protein coupling to RNA under irradiation with the UV light of 254 to $280 \mathrm{~nm}$ wavelength (Hockensmith et al., 1986). Naturally photo reactive nucleotide bases, primarily pyrimidines, can be coupled to several specific amino acids e. g. Phe, Trp, Tyr, Cys, and Lys (Brimacombe et al., 1988). It is also worth mentioning that the cross-linking of nucleic acids to proteins is not promoting any protein-protein crosslinking (Suchanek et al., 2005). Additionally, UV cross-linking requires a direct contact between molecules to be cross-linked, diminishing probabilities of false-positive results (Pashev et al., 1991).

Current research on protein-RNA cross-linking suggests that presence of affinity tags ( $\mathrm{e}$. g. His-tags) on a protein of interest greatly improves the speed and efficiency of cross-links recovery (Bohnsack et al., 2009). In accordance with this notion we have equipped Nb50 with a His-tag that allows purification of cross-links on a $\mathrm{Ni}^{2+}$ matrix under stringent purification conditions. To visualize cross-linked rRNA a 5' radiolabeling was performed, using $\gamma^{32} \mathrm{P}$-ATP and T4 polynucleotide kinase. The radiolabeled samples were then loaded 
onto an SDS-PAGE gel and visualized by radiography Figure 3.14. Additionally, western blot was done with anti His-tag antibodies to confirm the presence of Nb50 Figure 3.14.

A.

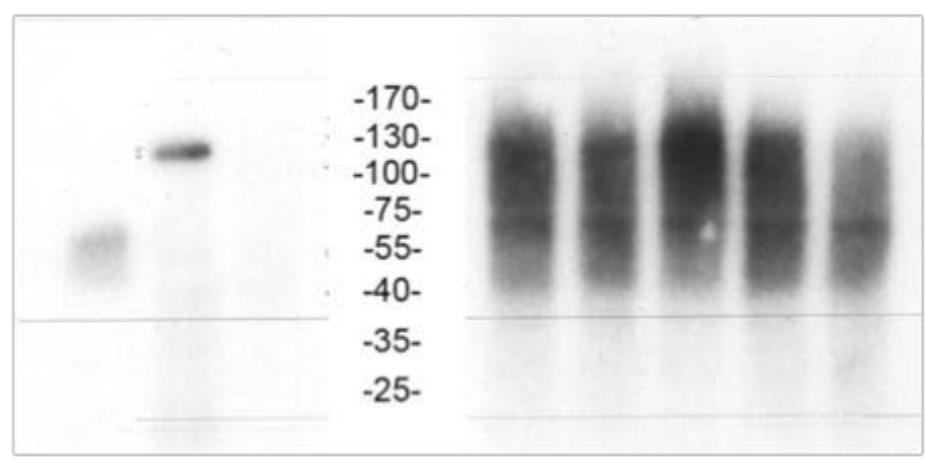

B.

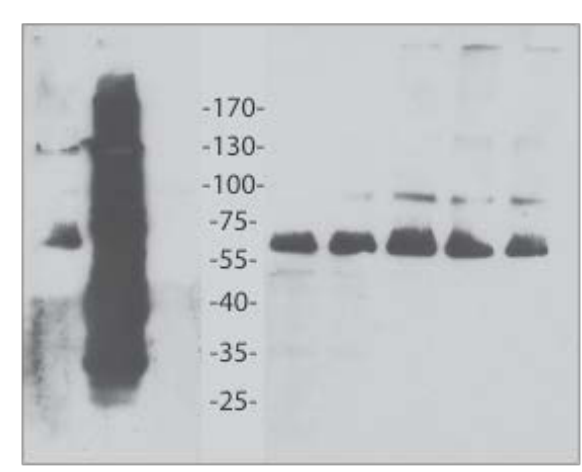

Figure 3.14 Nb50-rRNA cross-linking. (A) Radiolabeled rRNA-Nb50 cross-links visualized by autoradiography. The Nb50 was incubated with 50S ribosomal subunits from T. thermophilus and irradiated with UV light. The complex was then digested with an RNace. Upon immobilization of the complex onto a $\mathrm{Ni}^{2+}$ matrix, the cross-linked RNA was radiolabelled with $\gamma^{32} \mathrm{P}$-ATP. Then, the RNA-protein complexes were allowed to run on an SDS-PAGE gel and visualized by autoradiography. Lanes 4-8 correspond to Nb50-rRNA cross-links digested with different concentrations of RNace-IT. The complex was digested with the following RNace concentrations: $0.1,0.01,0.001,0.0001,0.00001 \mathrm{U}$ (lanes $4-8$ from left to right). Lane 1 represents a control where the $\mathrm{Nb50}$ was cross-linked to the total RNA extracted from $S$. cerevisiae. Lane 2 signifies a standard positive control where a yeast Prp43 protein was UV cross-linked to the total RNA extract from $S$. cerevisiae. And lastly a negative control with no $\mathrm{Nb50}$ can be appreciated on the lane 3 . (B) Western blot analysis of rRNA-Nb50 cross-links with anti-His-tag antibodies. The same membrane as seen on the left hand side (A) was treated with anti-His-tag antibodies to confirm the presence of the Nb50 in cross-linked samples. Lanes 4-8 correspond to the Nb50-rRNA cross-links treated with different RNace concentrations, a band running at around $60 \mathrm{kDa}$ corresponds to the $\mathrm{Nb} 50$. Lane 1 is the Nb50 cross-linked to the total RNA extracted from yeast. Lane 2 shows a standard positive control with Prp43, and finally lane 3 is a negative control where there is no $\mathrm{Nb} 50$ present. Cross-linking and further analysis was done by Prof. Bohnsack and colleagues.

Autoradiographic analysis of rRNA-Nb50 cross-links suggests that Nb50 indeed binds rRNA. Lanes 4-8 on the Figure 3.14 (A) represent Nb50-rRNA UV cross-links. Interestingly, the intensity of rRNA-Nb50 cross-links shows only marginal correlation with the amount of RNace used Figure 3.14 (A) Lanes 4-8. Only partial ribosomal RNA digestion by RNace $(0.1 \mathrm{U})$ might indicate that ribosomal proteins shield rRNA from RNace treatment. A set of appropriate controls was performed alongside the Nb50-rRNA cross-links. Prp43 is an RNA dependent ATPase (Arenas and Abelson, 1997) and it was used as a positive control because it binds yeast total RNA extract Figure 3.14 (A) Lane 2 (Bohnsack et al., 2009). Next, to test if Nb50 binds only rRNA from Thermus thermophilus or if it is more unspecific in terms of binding we have incubated Nb50 with 
total RNA extracted from S. cerevisiae. As can be seen in the Figure 3.14 lane 1, that Nb50 shows positive signal when incubated with the yeast total RNA.

To confirm that Nb50 co-migrates with the radiolabeled rRNA we performed a Western Blot analysis on the same membrane using anti-His-tag antibodies Figure 3.14 (B). Nb50 could be detected by antibodies recognizing the His-tag and it co-migrates with the radiolabeled rRNA.

After confirming that $\mathrm{Nb50}$ is a nucleic acid binder we wanted to identify the binding sites of $\mathrm{Nb} 50$ on the $50 \mathrm{~S}$ ribosome. To achieve this goal we have performed cross-linking and cDNA analysis (CRAC) experiment. CRAC relies on the deep sequencing of cDNA libraries generated from cross-linked RNA fragments. First, the concentration of RNace was optimized, so that the partial digestion of RNA leads to RNA pieces of detectable sizes. After sequencing of the cDNA library we have mapped the sequence reads to the Tth ribosomal RNA Figure 3.15. The most pronounced peak is a stretch of 23S rRNA between 2400 and 2470 bases. Analysis of the 50S structure showed that this rRNA stretch is exposed and could be accessed by the $\mathrm{Nb50}$.

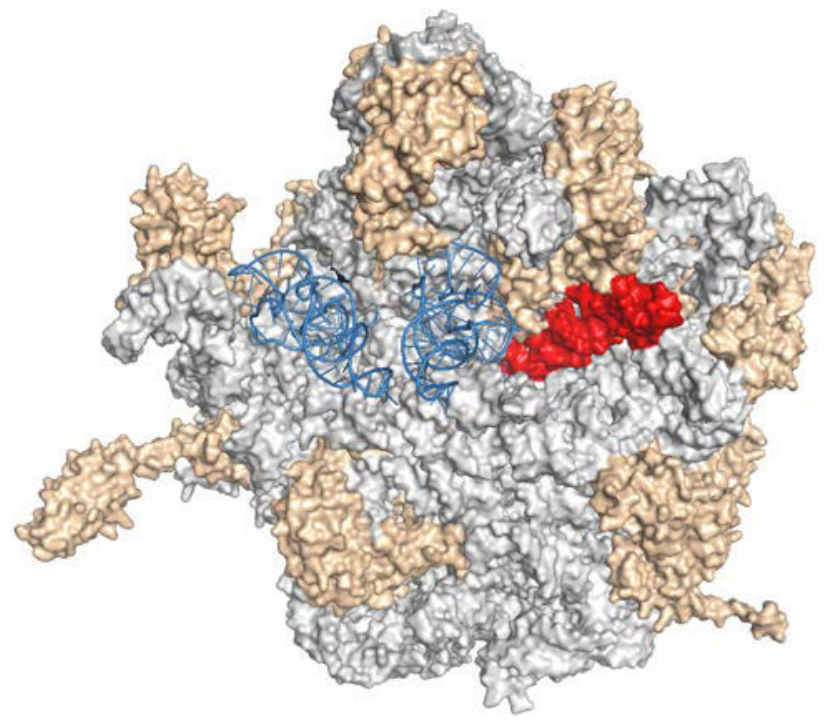

Figure 3.15 Nb50 rRNA binding site on the large ribosomal subunit. The binding site of $\mathrm{Nb50}$ was identified by CRAC. Nb50 was UV cross-linked to rRNA and subjected to the RNace and $6 \mathrm{M}$ guanidinium hydrochloride treatment. A cDNA library was constructed out of obtained cross-links. Next, the library was deep sequenced and the corresponding read-outs were mapped onto the rRNA of the Tth ribosomes. The Nb50 binding site is colored in red. tRNAse fitted in the P- and A-sites are colored in blue. Ribosomal proteins are in pale yellow, while rRNA is in gray. The experiment was performed by Prof. Bohnsack and his colleagues.

Interestingly, the identified rRNA epitope is a part of the A-site of the large ribosomal subunit. The A-site on the ribosome has a concave shape, which goes in line with the general belief that heavy-chain-only antibodies prefer to bind curvaceous pockets on antigens e. g. enzyme substrate pockets (De Genst et al., 2006). In future experiments we would like to further characterize the nature of the Nb50-rRNA interaction. Possible 
methods that could be used to study this interaction are cryo-EM or protein docking, although in the latter method the structure of the $\mathrm{Nb50}$ has to be determined first.

\subsubsection{Ribosomal subunit transport in FG-hydrogel permeation assay}

Transport receptors facilitate active translocation of molecules larger then $40 \mathrm{kDa}$ between the cytoplasm and the nucleus (Görlich and Kutay, 1999). There is a growing body of evidence that factors such as surface hydrophobicity of soluble proteins correlate with the cargo translocation competence. In addition, the number of nuclear transport receptors per cargo plays an important role in the kinatics of NPC passage, i. g. the higher the number of the transport receptors, the faster the NPC passage occurs (Ribbeck and Görlich, 2002; Schmidt and Görlich, 2015). Ribosomal subunits, reaching several mega-Daltons in size, are some of the largest cargoes in cells. They are also translocated by the NTRs, as shown by multiple studies that shed light onto the crucial role of NTRs in the process of ribosomal maturation and transport (Trotta et al., 2003; Wu et al., 2016; Yao et al., 2007). Despite great progress in the identification of ribosomal NTRs, the minimal number of NTRs necessary for a successful transport event of ribosomal subunits was not established. We wanted to address this question by using our anti-ribosomal nanobodies. In order to recruit NTRs to an exogenous ribosome (i. e. a ribosome that does not contain NES/NLS, which can be recognized by the host cell), we designed an experimental approach in which a NES or NLS has to be fused to Nbs binding Tth ribosomes. In other words, NES/NLStagged Nbs serve as anchor points where nuclear transport receptors can bind, thus bridging them with ribosomal subunits. To test the permeability properties of ribosomes decorated with NTRs we performed the FG particles assay (Schmidt and Görlich, 2015). FG particles assay offers several critical advantages over other testing methods including ease and speed of testing as well as ability to test different FG Nups (H. B. Schmidt, 2015). First, we have equipped nanobodies with super NES that can be recognized by CRM1 (Güttler et al., 2010). Unfortunately, NES fused to nanobodies was prone to degradation when being expressed in E. coli (data not shown). To circumvent this problem we fused $\operatorname{Imp} \beta \mathrm{N}$-terminally to 6 orthogonal nanobodies. In this case, $\operatorname{Imp} \beta$-nanobody fusions were well expressed and soluble. For FG particles assay $\operatorname{Imp} \beta$ fused nanobodies were premixed with fluorescently labeled ribosomes, where $\mathrm{Nbs}$ were in 2-fold excess over the concentration of ribosomes. As can be seen on the Figure 3.16, 50S ribosomal subunits in the absence of any NTRs predominantly reside in the solution surrounding the FG 
particles. Upon addition of $\operatorname{Imp} \beta$ alone, however, 50S ribosomes enter FG particles Figure 3.16. This unexpected observation suggests that human $\operatorname{Imp} \beta$ can have an affinity towards the $50 \mathrm{~S}$ ribosomal subunits from $T t h$.

FG particles challenged with 50S ribosomal subunits

No ImpB

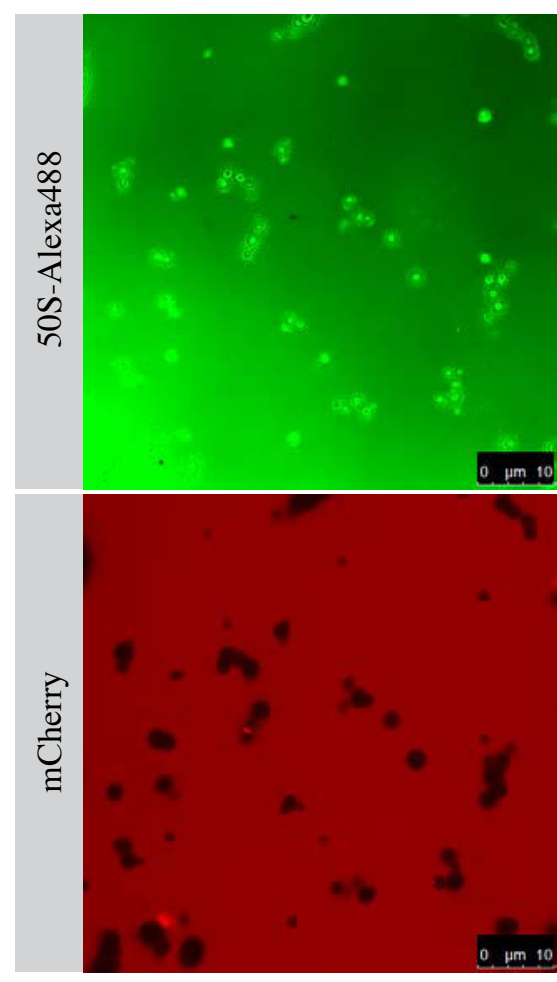

$\operatorname{ImpB}$

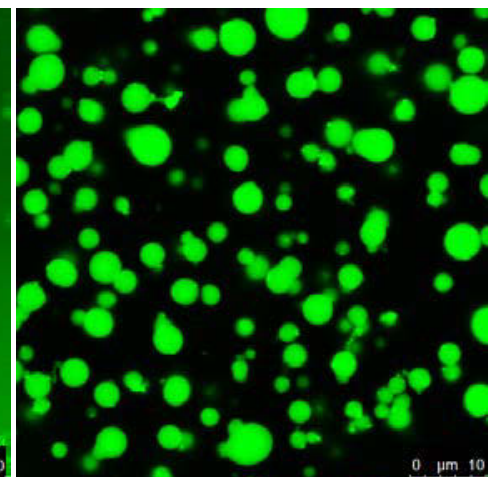

Nb46-Imp B

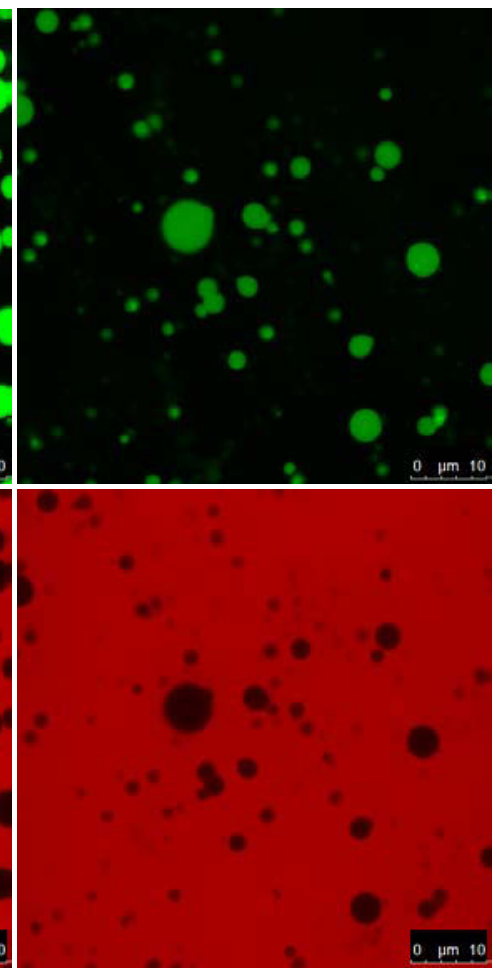

Figure 3.16 Effect of Impß on the entry of 50S ribosomal subunits into FG particles formed by Nup116. 50S-Alexa488 ribosomal subunits were pre-mixed with $\operatorname{Imp} \beta$ or $\mathrm{Nb} 46-\operatorname{Imp} \beta$ and incubated for $1 \mathrm{~h}$. The complex was added to FG particles and incubated for $1 \mathrm{~h}$ and finally imaged at a confocal microscope. The negative control can be seen in the left hand image, where no $\operatorname{Imp} \beta$ was added. 50S-Alexa488 incubated with $\operatorname{Imp} \beta$ center. The 50S labeled with Alexa 488 were incubated in the presence of $\mathrm{Nb} 46-\operatorname{Imp} \beta$.

$\operatorname{Imp} \beta$ is reported to be responsible for the active translocation of ribosomal proteins into the nucleus (Jäkel and Görlich, 1998). As reported by Jäkel and Görlich, 1998, Imp $\beta$ binds a very basic region on r-proteins and it binds ribosomal proteins directly without its partner Imp $\alpha$. we therefore conclude that it can not be ruled out that $\operatorname{Imp} \beta$ can bind ribosomal proteins even when they are in complex with rRNA, as most of ribosomal proteins are very basic. Another explanation of this experiment could be that fluorescently labeled ribosomal proteins dissociate and together with $\operatorname{Imp} \beta$ migrate into FG particles.

The propensity of $\operatorname{Imp} \beta$ to bind ribosomal proteins posed several limitations on the given experimental approach, also requiring new NTRs to be tested. 


\subsection{Generation of nanobodies against lamin isoformes from Xenopus laevis}

Lamins are intermediate filaments that provide both the structural support and the functional regulation to the nucleus. Lamins are present in all metazoan cells and, although sequences of lamins might differ significantly, their general structural organization is conserved. In this study we focus on lamin B3, that is a major lamin isoform in Xenopus laevis oocytes and eggs (Benavente et al., 1985). We aim at the generation of anti-lamin B3 nanobodies, which can be used for imaging, biochemistry and structural analysis of lamin B3.

\subsubsection{Recombinant expression of lamins in E. coli}

First, we set out to obtain lamins in a soluble form for the immunization of alpacas and for the further bio-selection of nanobodies. Typically, when intermediate filaments are expressed in E. coli they tend to polymerize and precipitate, thus forming inclusion bodies. As a result, the standard method of lamin purification is protein recovery from inclusion bodies (Bollati et al., 2012; Moir et al., 1991; Strelkov et al., 2004). The process of obtaining the protein of interest from inclusion bodies, however, is labor intense and results in low protein yields. Therefore, we sought after protein expression tags that might help improving the solubility of lamins. As a protein fusion partner, we have chosen IF2 1158, a domain of the bacterial translation initiation factor IF2 that was reported to improve protein solubility in a manner similar to MBP and NusA (Sørensen et al., 2003). IF2 1-158 fused to lamin B3 has increased solubility compared with the otherwise insoluble lamin B3 Figure 3.17. 


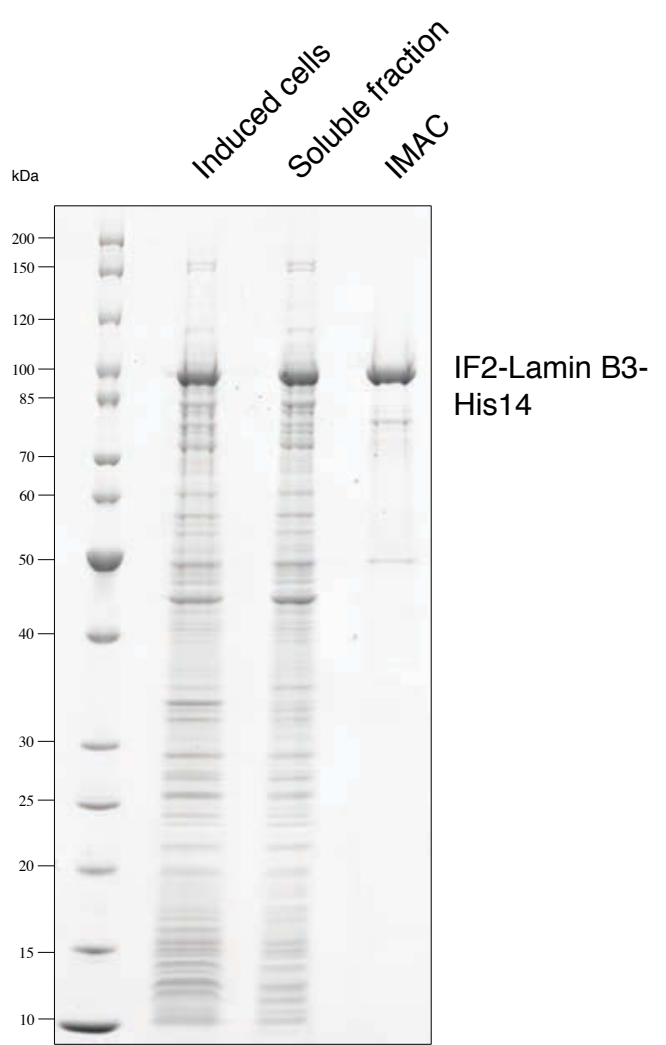

Figure 3.17 Purification of recombinantly expressed lamin $\mathbf{B 3}$ from $\boldsymbol{E}$. coli. NEB Express cells were transformed and grown overnight, then a single colony was picked and pre-grown overnight at $27^{\circ} \mathrm{C}$ until $1 \mathrm{OD}$ was reached. Then, the cells were induced with IPTG and grown for $5 \mathrm{~h}$ at $23^{\circ} \mathrm{C}$. The culture was harvested, sonicated and ultracentrifuged. The soluble fraction was purified on $\mathrm{Ni}^{2+}$ matrix and eluted with imidazole. Samples of induced culture, soluble fraction, and purified lamin B3 (IMAC) were analyzed on a SDS-PAGE gel.

In order to obtain lamin in its soluble, tag-free form, we have established a purification scheme with MBP fusion domains Figure 3.18.

Purification of Lamin B1 from E. coli

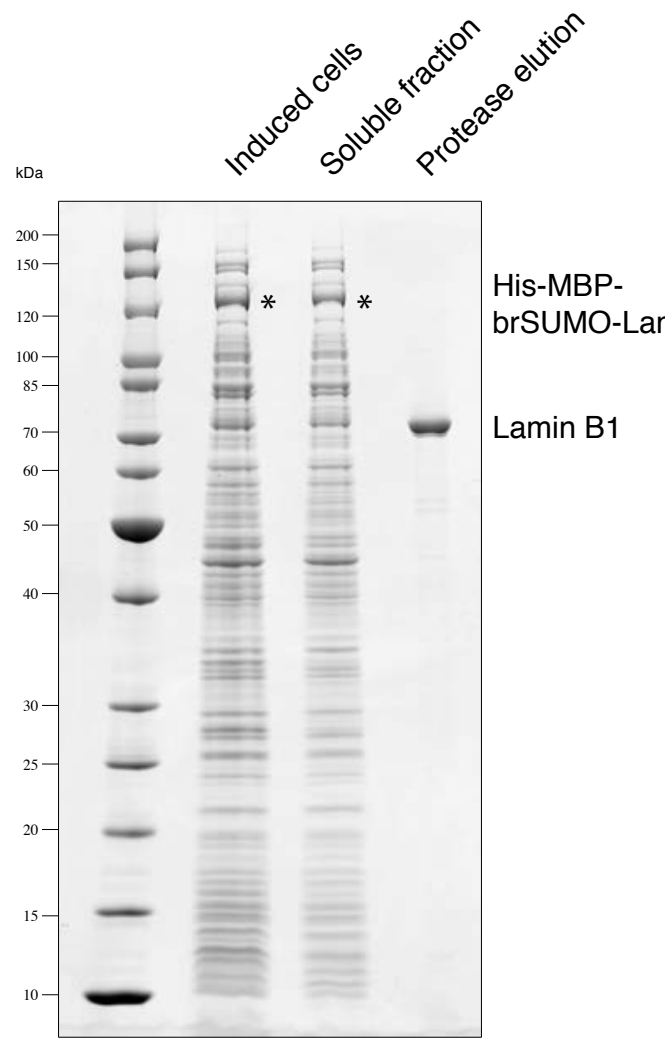

Purification of Lamin B2 from E. coli

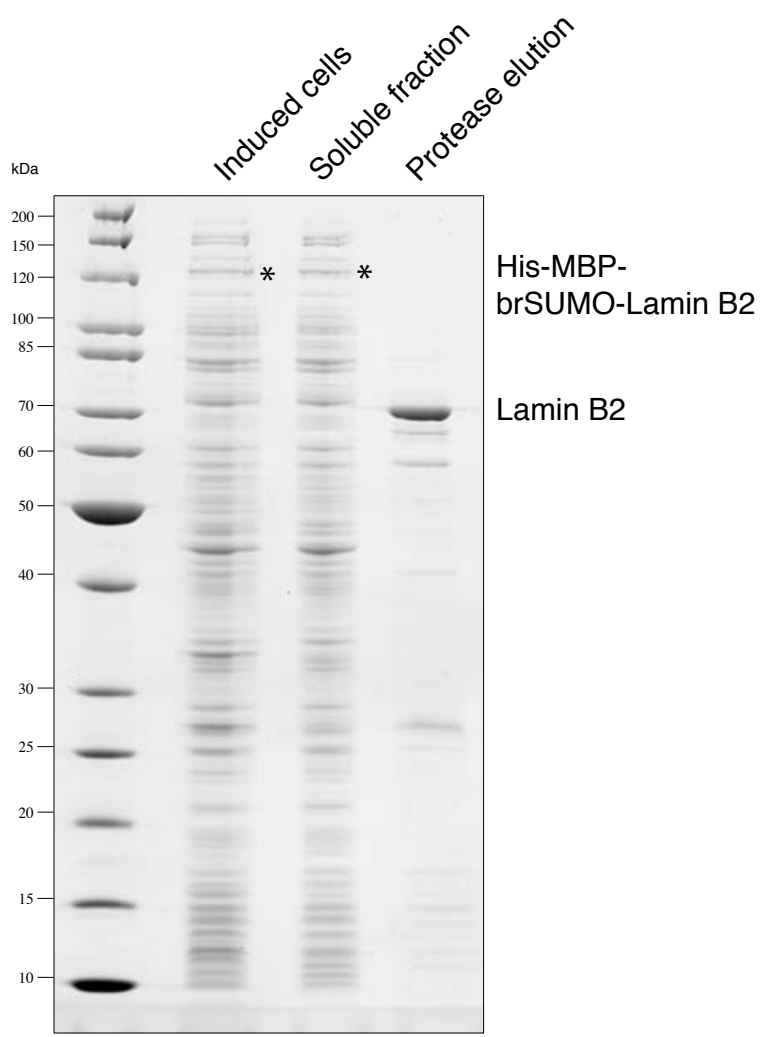


Figure 3.18 Purification of $X$. laevis lamin isoforms B1 and B2 from $E$. coli in a tag-free form. A sample of induced cells and of soluble material were analyzed on an SDS gel and stained with Coomassie. Note, the asterisk signifying the soluble lamin. The soluble fraction was loaded onto a $\mathrm{Ni}^{2+}$ column, washed with imidazole and eluted with SUMO protease.

Expressing lamin in E. coli recombinantly we were able to obtain approximately $10 \mathrm{mg}$ of pure protein from one liter of culture.

\subsubsection{Selection of nanobodies against lamin B3}

Nanobodies against lamin B3 were selected as described in the "Materials and methods" section. In short, after 3 rounds of selection nanobodies were re-cloned into the characterization vector. 56 nanobodies were sequenced and aligned into a phylogenetic tree using Ugene software (Okonechnikov et al., 2012). For further characterization and analysis we have chosen 11 representative nanobodies from different classes Figure 3.19.

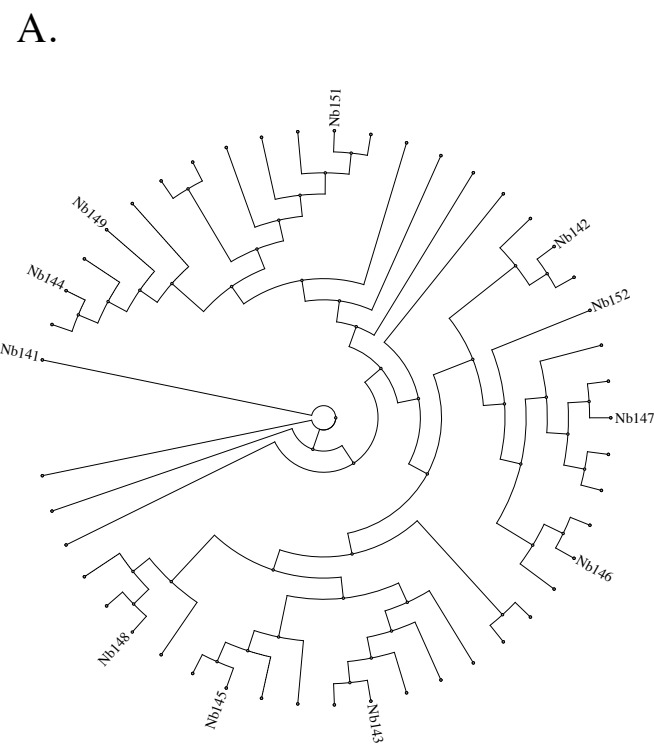

B.

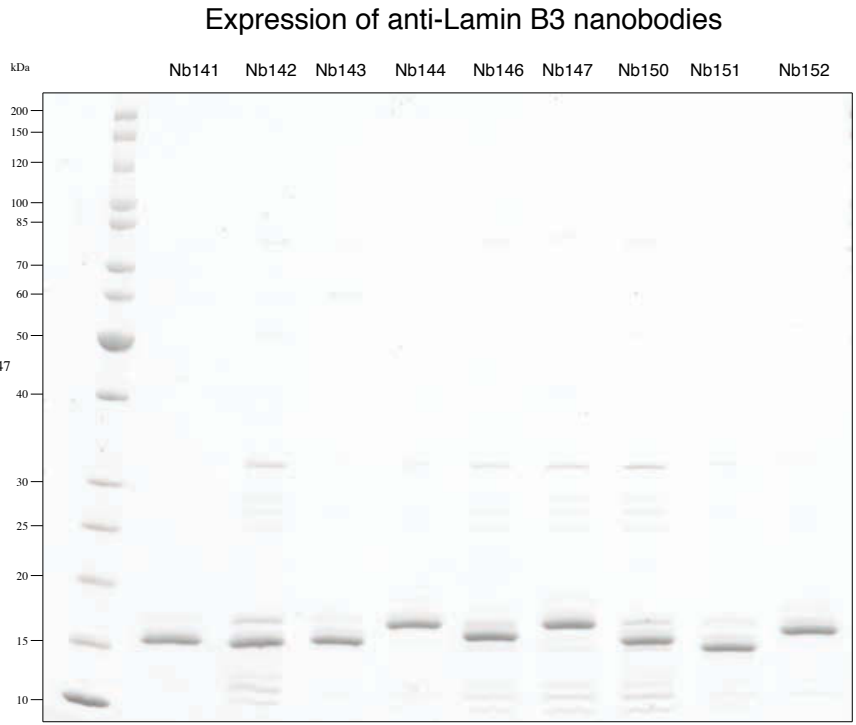

Figure 3.19 Phylogenetic tree of anti-lamin B3 nanobodies and expression of selected nanobodies. (A) The phylogenetic tree with selected nanobodies highlighted. (B) Selected nanobodies expressed in a characterization vector (His14-Avi-tag-brSUMO-Nb) for further analysis.

All expressed nanobodies showed high solubility and good expression yields. Thus, we have chosen to test all of them if they can be used for isolation of lamin B3 and microscopy. 


\subsubsection{Isolation of recombinantly expressed lamin B3}

To characterize selected nanobodies we have expressed eleven nanobodies in the following cassette His14-AviTag-brSUMO-Nb. The presence of the Avi-tag allowed a fast and specific nanobody capture on streptavidin magnetic beads. Tested nanobodies exhibited the ability to bind recombinant lamin B3 Figure 3.20. Next, we wanted to analyze if affinity purified lamin B3 was able to form paracrystals, which is a characteristic feature of lamins in vitro (Karabinos et al., 2003).
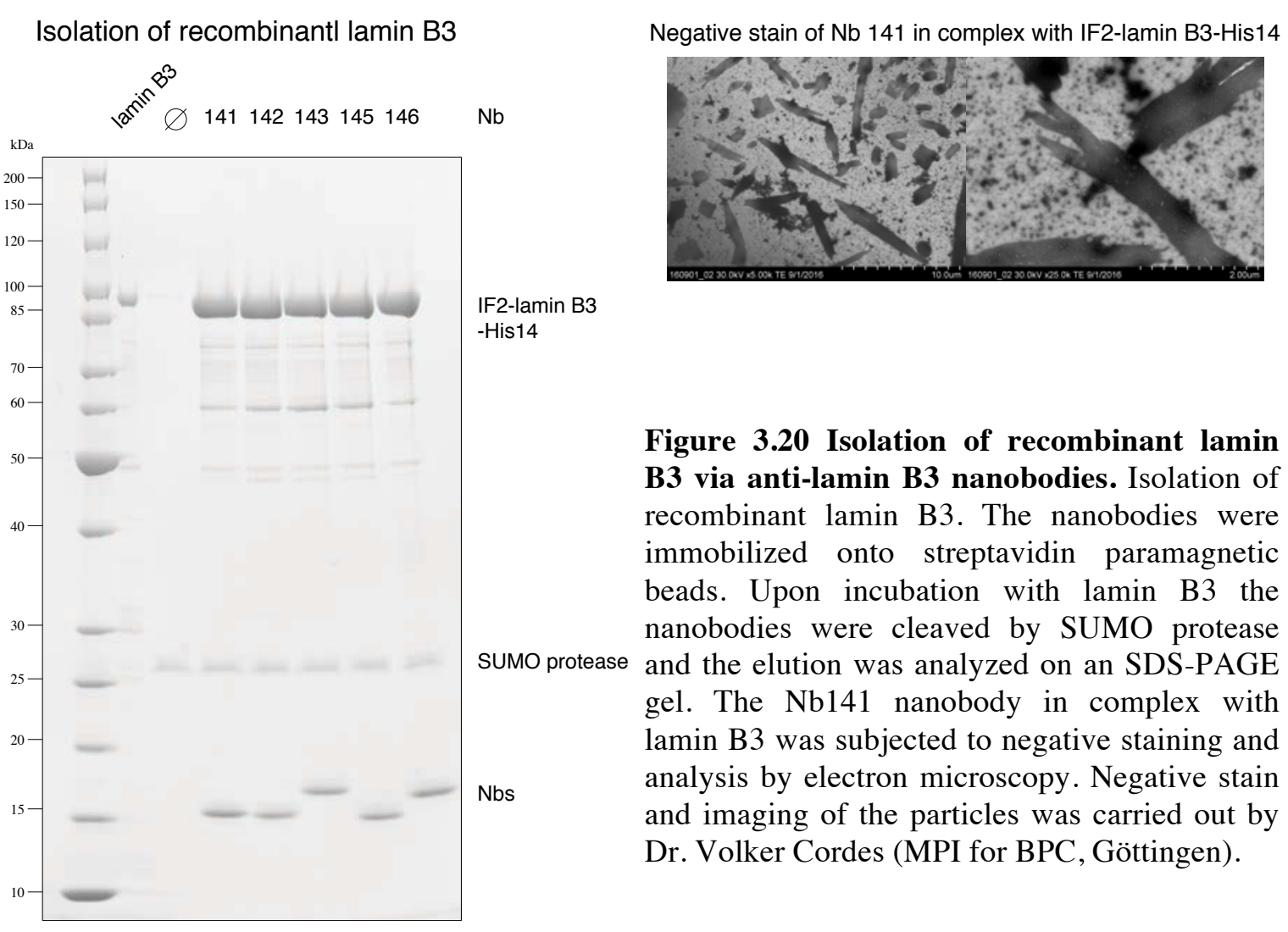

Figure 3.20 Isolation of recombinant lamin $B 3$ via anti-lamin B3 nanobodies. Isolation of recombinant lamin B3. The nanobodies were immobilized onto streptavidin paramagnetic beads. Upon incubation with lamin B3 the nanobodies were cleaved by SUMO protease and the elution was analyzed on an SDS-PAGE gel. The Nb141 nanobody in complex with lamin B3 was subjected to negative staining and analysis by electron microscopy. Negative stain and imaging of the particles was carried out by Dr. Volker Cordes (MPI for BPC, Göttingen).

The negative stain reveals that lamin B3 in the IF2-lamin B3-His construct is able to form high order assemblies. The size of the paracrystals ranges from a few micrometers up to hundreds of micrometers. Firstly, the formation of paracrystals could indicate that the IF2 expression tag does not influence behavior of lamin B3 through posing steric hindrances. Secondly, the fact that the lamin B3 paracrystals are present in the nanobody eluted fractions could argue that the tested nanobodies are able to bind the polymerized form of lamin. In addition, these nanobodies do not seem to cause disassembly of lamin B3 paracrystals. 


\subsubsection{Identification of nanobodies binding sites on lamin B3}

An important aspect in the characterization of any antibody is the identification of its binding site. There are several methods available to study the epitopes of an antibody, such as structural methods, mass spectrometry, or generation of antigen truncations. Based on the established method to express lamin B3 recombinantly in a soluble form, we have made a use of the IF2 and MBP expression systems to generate a head, a rod, and a tail domain of lamin B3. The goal of this approach was to test via immunoprecipitation which epitopes of lamin B3 the nanobodies bind Figure 3.21.

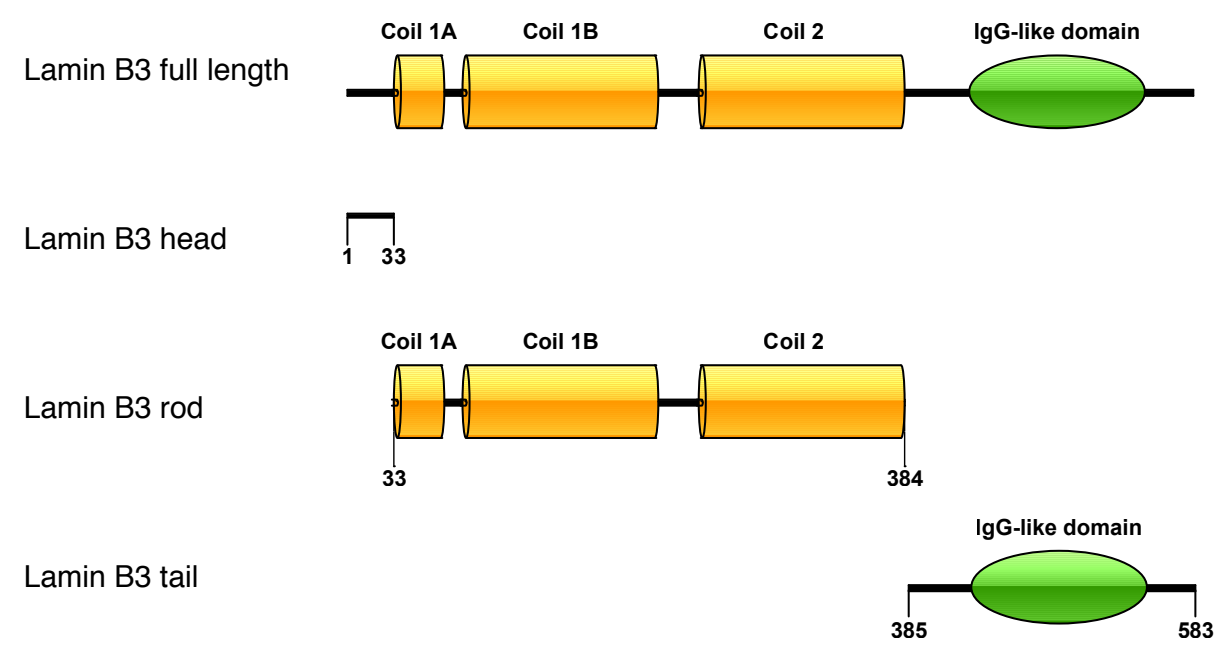

Figure 3.21 Schematic representation of lamin B3 fragments. Lamin B3 truncations were expressed with an $\mathrm{N}$-terminal IF2-domain and a C-terminal His-tag. The drawings were made using IBS software (Liu et al., 2015).

The design of the given lamin B3 truncations was based on the different functions carried by the respective domains, as well as their structural differences. The first truncation is the head domain, which was shown to contain conserved phosphoacceptor site that are conserved among lamin isoforms. Phosphorylation of the lamin head domain by the cdc2 kinase is a critical step for the longitudinal association of lamin molecules, underscoring the functional importance of this domain (Heald and McKeon, 1990). The second truncation contains the rod domain, that was demonstrated to be responsible for the dimerization properties of lamin (Schirmer et al., 2001). The last truncation contains the tail domain. It carries a nuclear localization signal and the IgG-like domain that plays role in formation of lamin higher order assemblies (Shumaker et al., 2005). All these lamin B3 fragments were well expressed and soluble Figure 3.22. 
A. Lamin B3 fragments

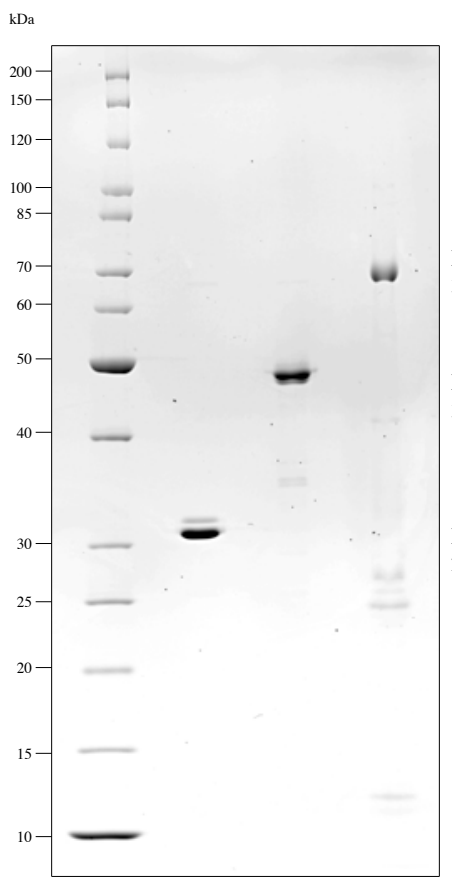

C.

Nanobody 141 pull-down of Lamin B3 fragments
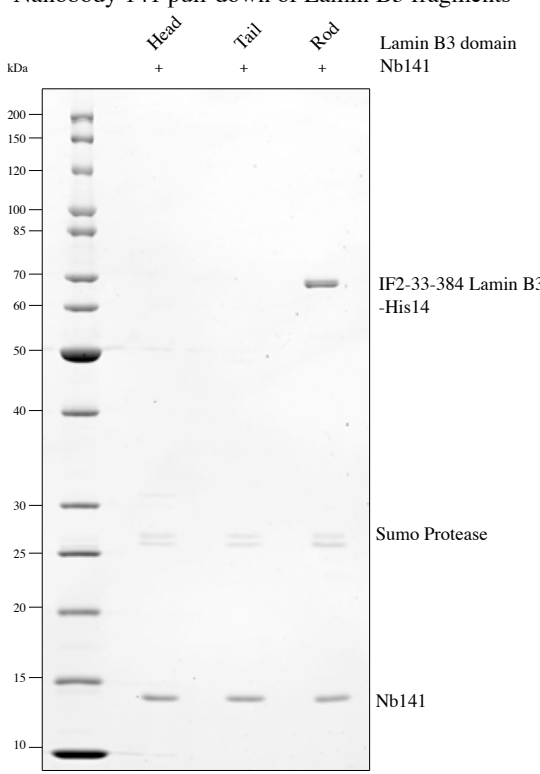

B. Nanobody 152 pull-down of Lamin B3 fragments

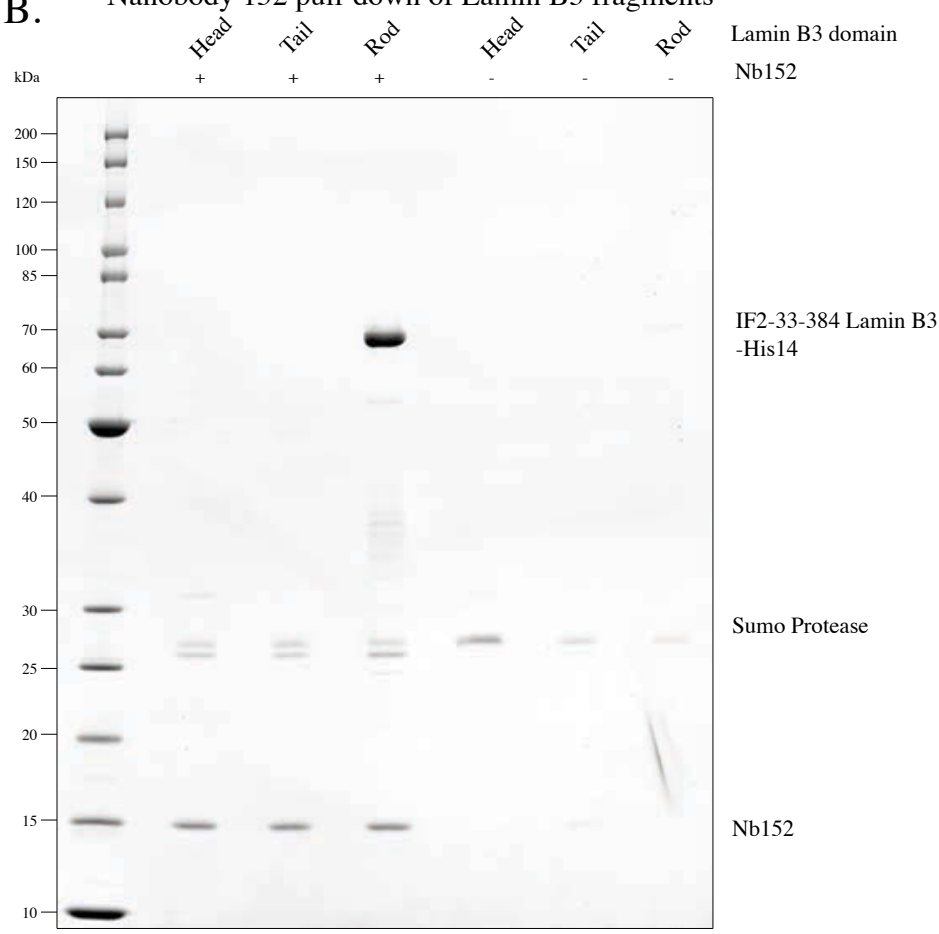

D.

Pull down of recombinant Lamin B3 fragments via immobilized nanobodies

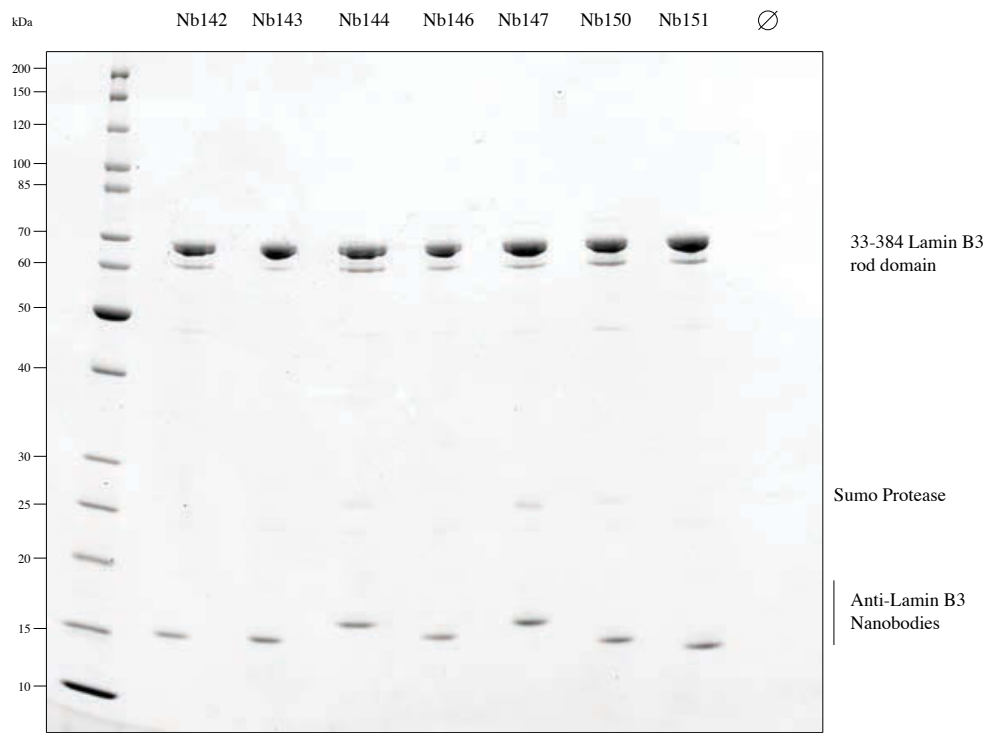

Figure 3.22 Generation of lamin B3 truncations (A). Lamin B3 truncations were designed based on the lamin B3 functional domains. The head, rod, and tail domains were expressed as fusions with the IF2 domain and purified on $\mathrm{Ni}^{2+}$ IMAC. Identification of nanobodies binding lamin $\mathrm{B} 3$ truncations was performed (B-D). Expressed truncations were used as a prey to be captured by immobilized nanobodies. The captured lamin B3 truncations were eluted by addition of the SUMOstar protease. Eluted lamin-Nb complexes were mixed with the SDS loading buffer and analyzed on SDS-PAGA gels, stained by the Coomassie dye.

The isolation of lamin B3 truncated mutants shows that all nine nanobodies recognize the lamin B3 rod domain region. This fact can speak in favor of the rod domain higher 
immunogenicity overall in the lamin B3 molecule. Given results could be also explained by the preferences of nanobodies to bind structured protein domains. The rod domain is composed of alpha-helical domains, which become structurally more rigid when laterally polymerized, this fact could have led to the selection of nanobodies that are targeting the polymeric forms of lamin B3.

We established that nanobodies $\mathrm{Nb} 141$ and Nb152 are orthogonal to each other (see paragraph 3.2.5 for the details), thus, we set out to test if these nanobodies bind different epitopes on the rod domain. As the rod domain has several coiled-coil domains we have generated lamin B3 33-215 and lamin B3 216-384 mutants in order to test putative binding sites of the aforementioned nanobodies. The immunoprecipitation experiments show that the $\mathrm{Nb} 141$ and $\mathrm{Nb} 152$, although orthogonal, bind to the same structural region of lamin B3, namely amino acids 33 to 215 Figure 3.23 .

A.

Nanobody 141 pull-downs of Lamin B3 mutants

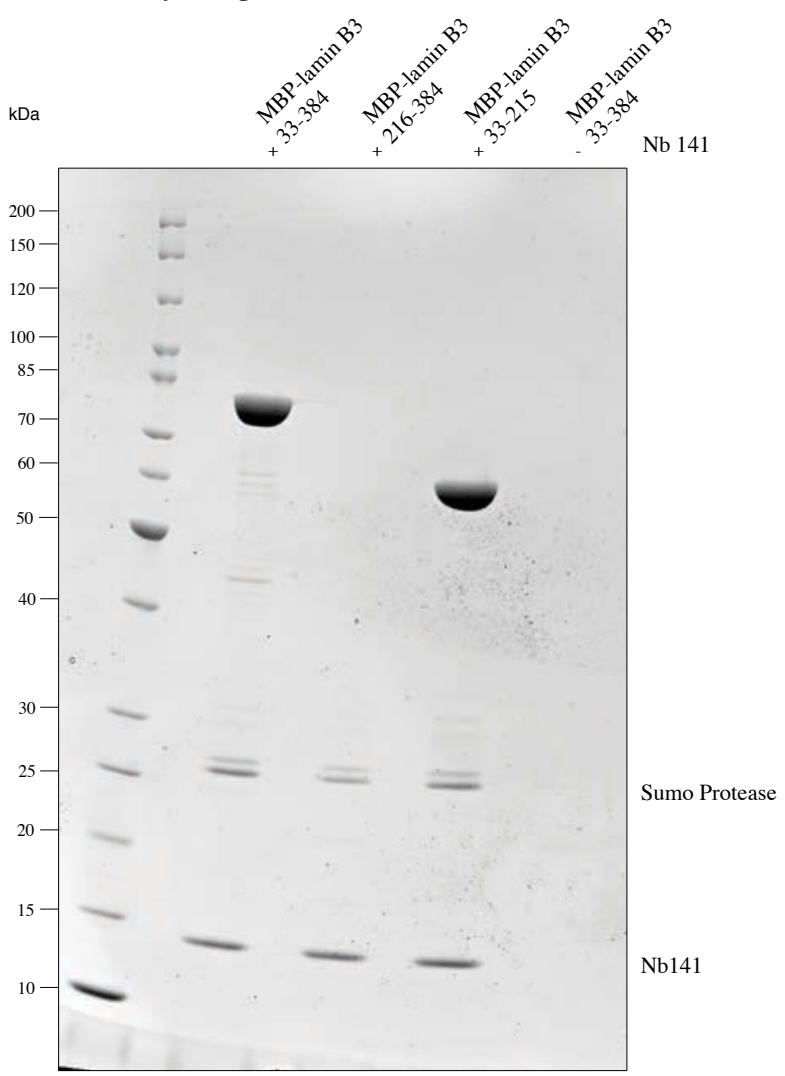

B.

Nanobody 152 pull-downs of Lamin B3 mutants

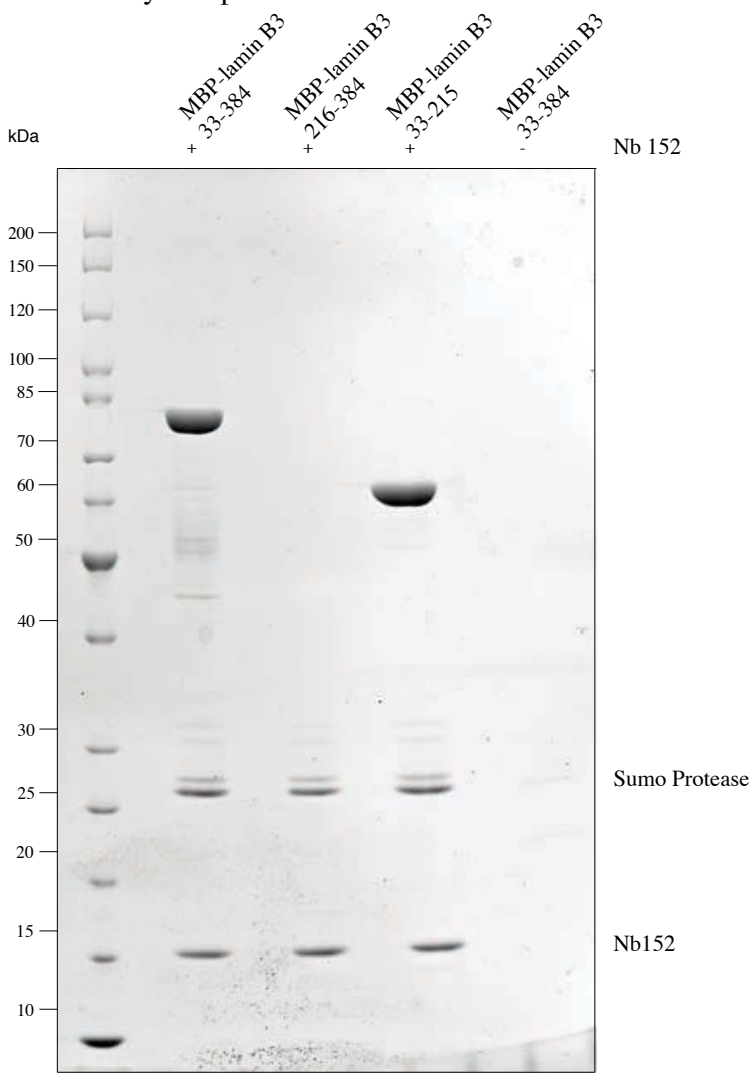


C.

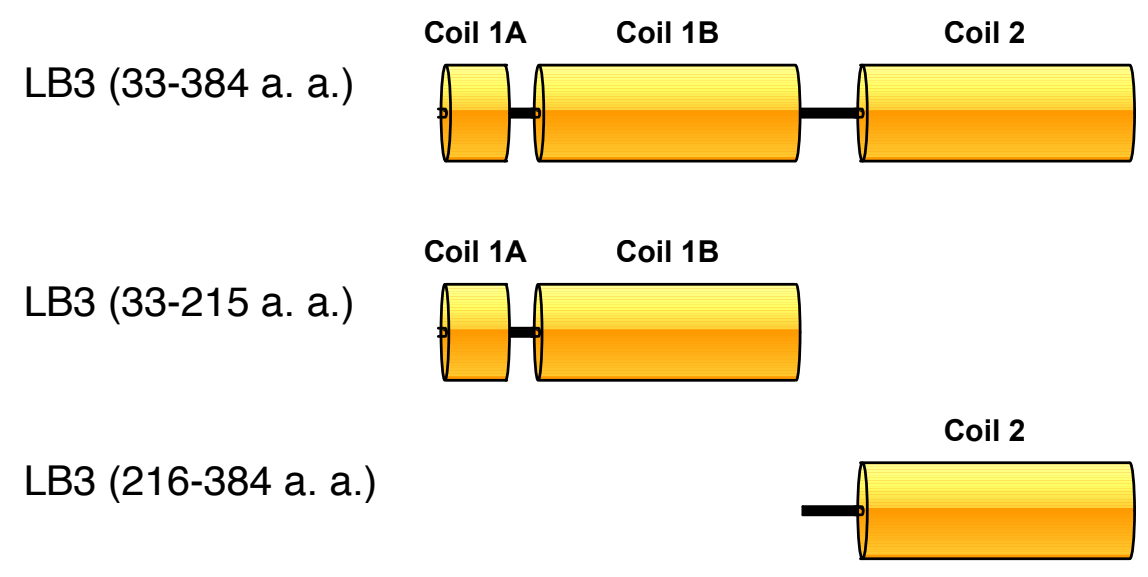

Figure 3.23 Identification of Nb141 and Nb152 binding sites on lamin B3. (A, B). The orthogonal pair Nb141 and Nb152 was immobilized onto MyOne Streptavidin T1 magnetic beads and incubated with purified lamin B3 rod domain truncations. The nanobodies were eluted by SUMO protease. Eluted samples were mixed with the SDS loading buffer and analyzed on an SDSPAGE gel, followed by Coomassie staining. (C) The lamin B3 rod truncations were expressed in $E$. coli and purified on IMAC. The pictures were drown using IBS software (Liu et al., 2015).

\subsubsection{Orthogonal anti- lamin B3 nanobodies}

The next step in the characterization of anti- lamin B3 nanobodies was to establish if there are orthogonal binders available in the selected nanobody pool. The availability of orthogonal nanobodies against the lamin B3 could be beneficial in several ways. First, orthogonal variants could be used together to increase the fluorescent signal in imaging. Second, an experimental design can be envisaged in which one nanobody could be used as a vehicle to attach drug/inhibitor to lamin that influence its function, while the other nanobody could be used to visualize the lamina. To test possible orthogonality of nanobodies we have designed several appropriate constructs for nanobody expression that have different molecular weights. These constructs can be appreciated in the Figure 3.24.
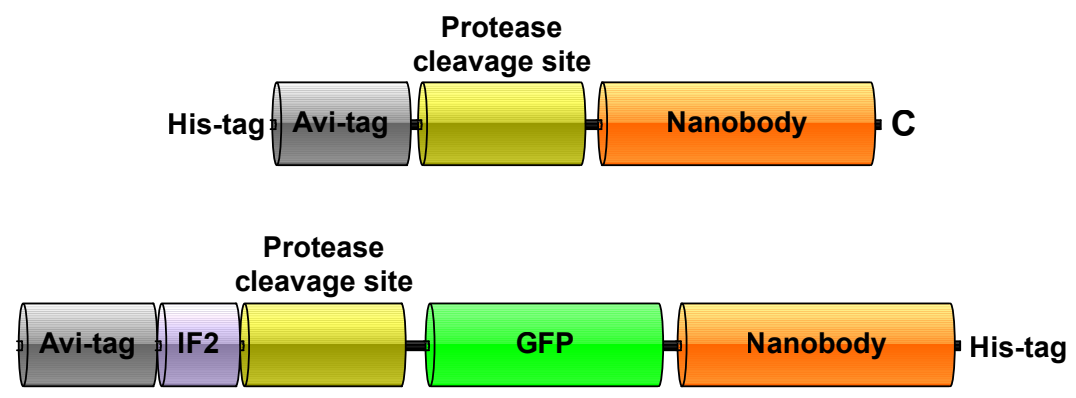

Figure 3.24 Open reading frames of nanobody characterization vectors for the orthogonality tests. 
As we previously established that all selected nanobodies bind the rod domain of lamin B3 33-384, we decided to use it for the orthogonal tests of selected nanobodies Figure 3.25.

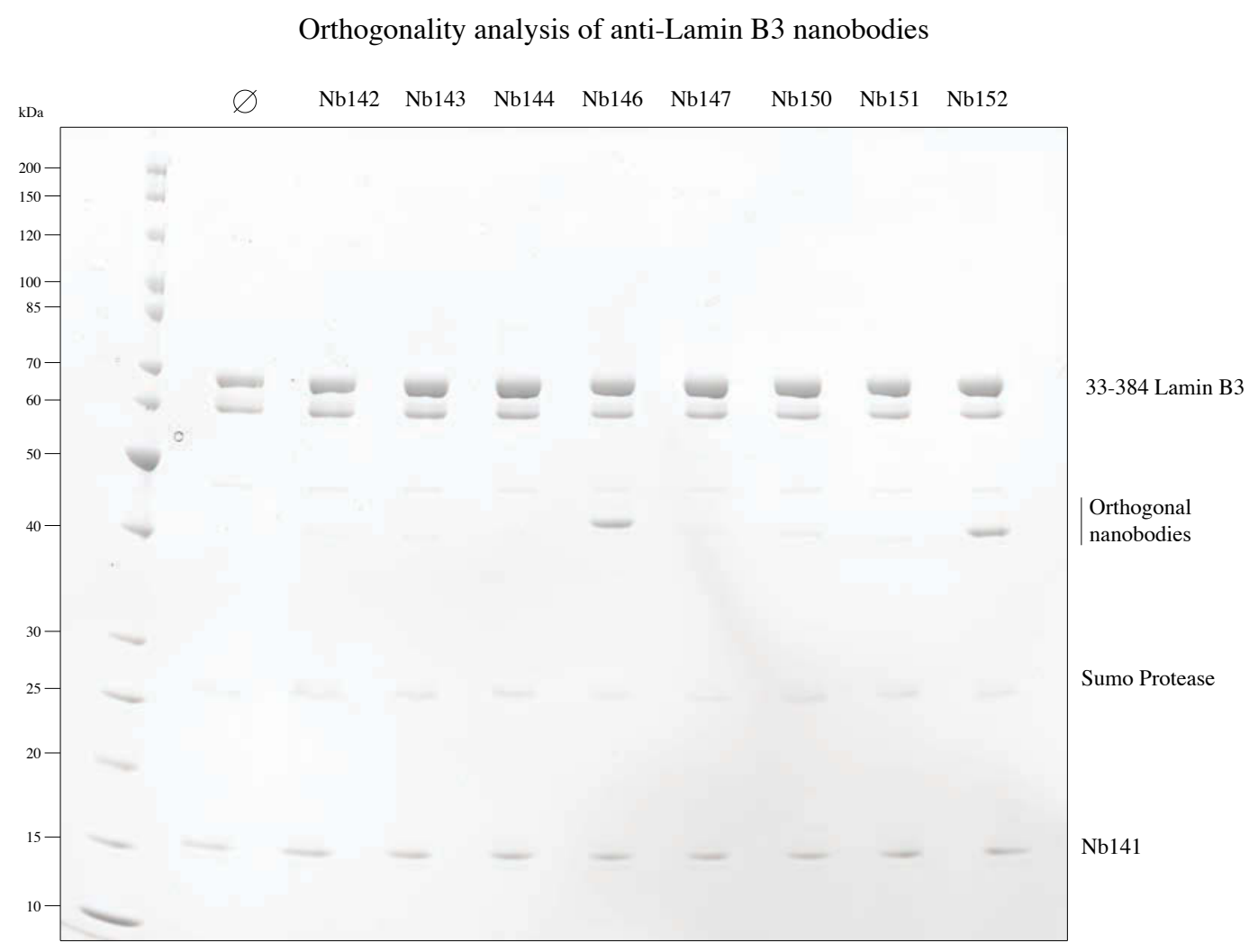

Figure 3.25 Assessment of anti- lamin B3 nanobodies orthogonality via affinity immobilized lamin B3 33-384 binding. Nanobodies Nb146 and Nb152 are orthogonal to Nb141. To identify orthogonal lamin B3 nanobodies a single variant was immobilized onto a support via the Avi-tag and incubated with the lamin B3 33-384 mutant, then the nanobody-GFP fusions were added to the reaction. Formed complexes were washed and eluted with the SUMO protease and analyzed on SDS-PAGE gels.

As can be seen in the Figure 3.25, there are two nanobodies that are orthogonal to the Nb141. Considering that the band intensities of both nanobodies are comparable, we can conclude, that the respective orthogonal nanobodies have different binding sites on lamin B3, which likely do not overlap.

Therefore, based on the orthogonality tests we have selected nanobodies 141 and 152 for further characterization.

\subsubsection{Specificity of selected orthogonal nanobodies against different lamin isoforms}

Lamin $b$ gene is present ubiquitously among all metazoans, in at least one single copy and is essential for the viability of the cell (Ellis et al., 1997). Several lines of evidence support 
the notion that all lamins evolved from a single lamin type b progenitor (Stick, 1992). As a result of gene duplication vertebrates posses two lamin b isoforms. In Xenopus laevis, however, there are three lamin b genes Figure 3.26, where lamin B3 is a major isoform expressed in oocytes.

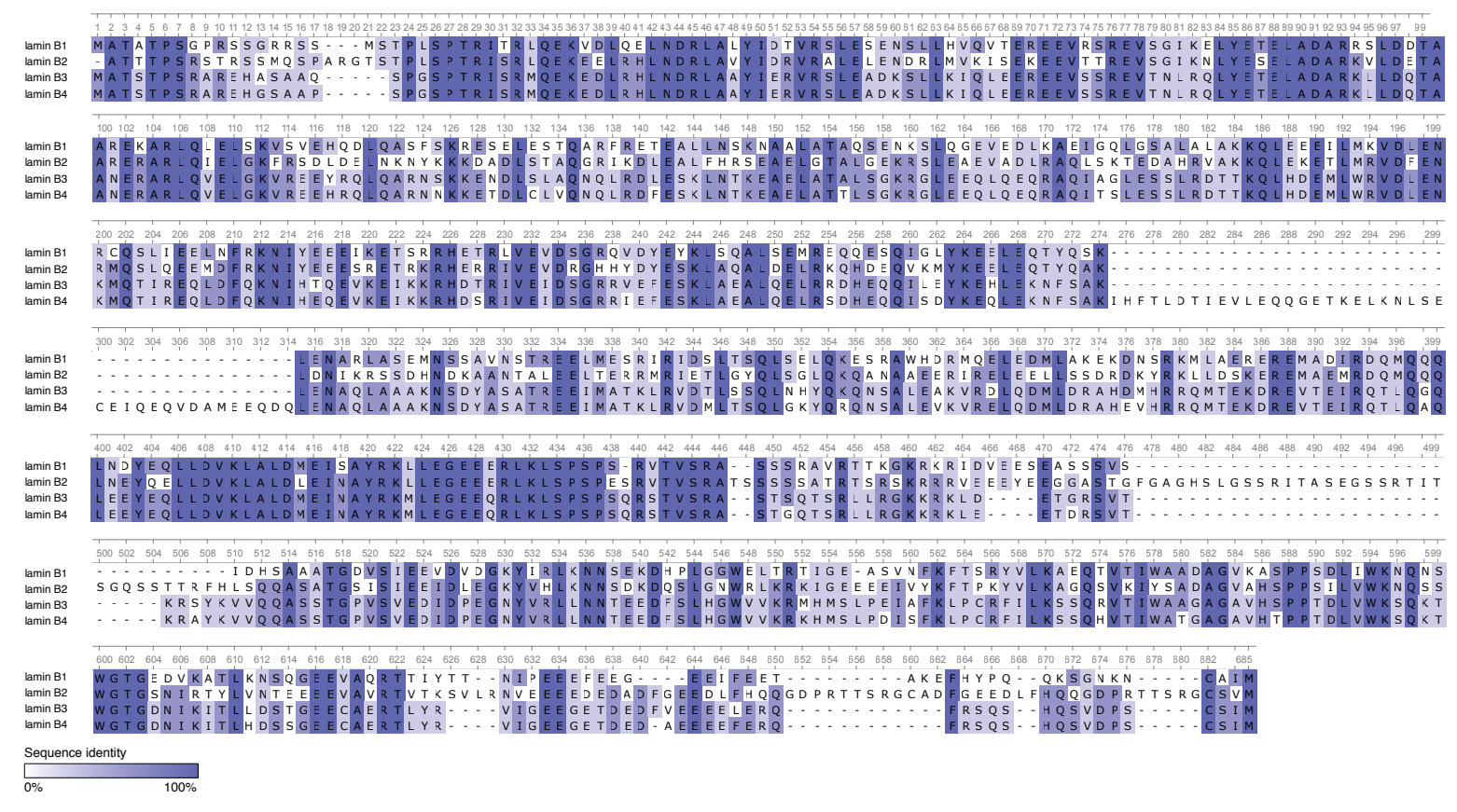

Figure 3.26 Comparison of protein sequences of four major lamin type $B$ isoforms. Lamin $B$ type isoforms B1, B2, B3, B4 from X. laevis were aligned according to the amino acid similarities in their protein sequences. The lamin type B isoforms are aligned using Ugene alignment tool (Okonechnikov et al., 2012). Note the sequence identity bar.

The lamin B3 and B4 isoforms are essentially two splice isoforms of LIII $\beta$ gene. Alternative splicing of the $L I I I \beta$ gene results in an insertion of 40 amino acids into the $2 \mathrm{~B}$ coiled-coil domain.

X. laevis lamin B3 isoform shares about 50\% similarity with lamins B2 and B1. In this light we wanted to investigate if the selected orthogonal nanobodies are lamin B3 isoform specific or if they also recognize other lamin B type isoforms. Lamin B1 and B2 isoforms were expressed recombinantly in E. coli. To obtain the respective isoforms in a tag-free form they were cloned into the following expression cassette: His14-MBP-SUMO-Lamin. The MBP expression module allows an expression with high yields and the SUMO cleavage module allows for protease elution of the expressed lamins in a tag-free form. Recombinantly expressed lamin isoforms were used as preys in the affinity purification assays with immobilized nanobodies Nb141 and Nb152 Figure 3.27. 
Affinity chromatography of recombinant $X$. laevis lamin B1, B2, B3 isoformes via Nb 152

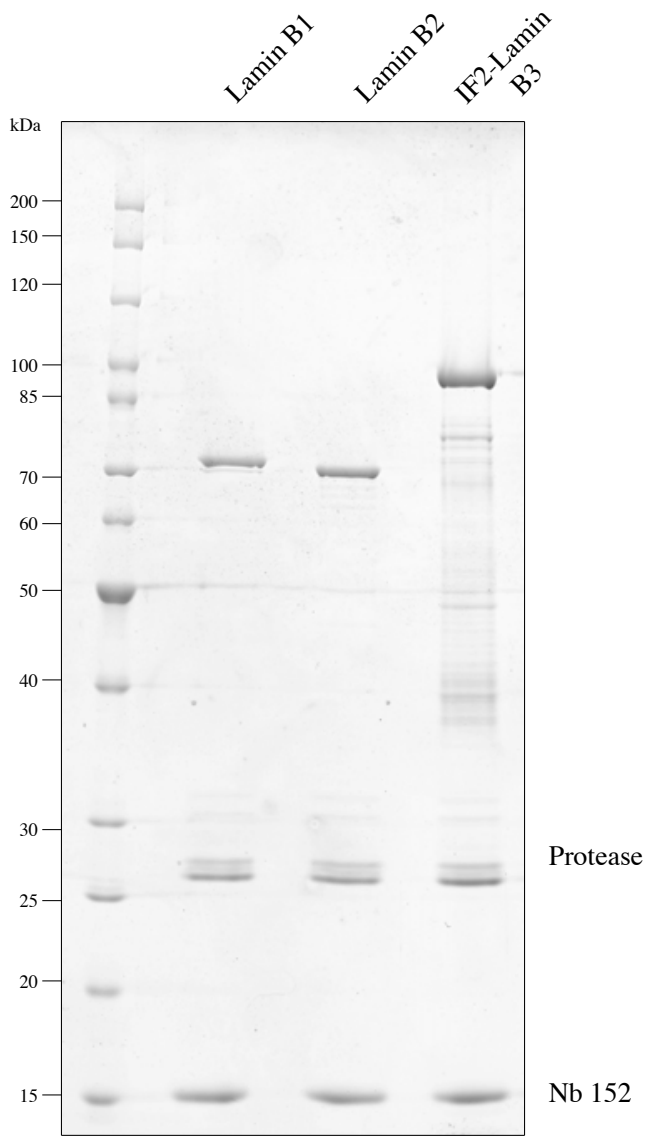

Affinity chromatography of recombinant $X$. laevis lamin B1, B2, B3 isoformes via Nb 141

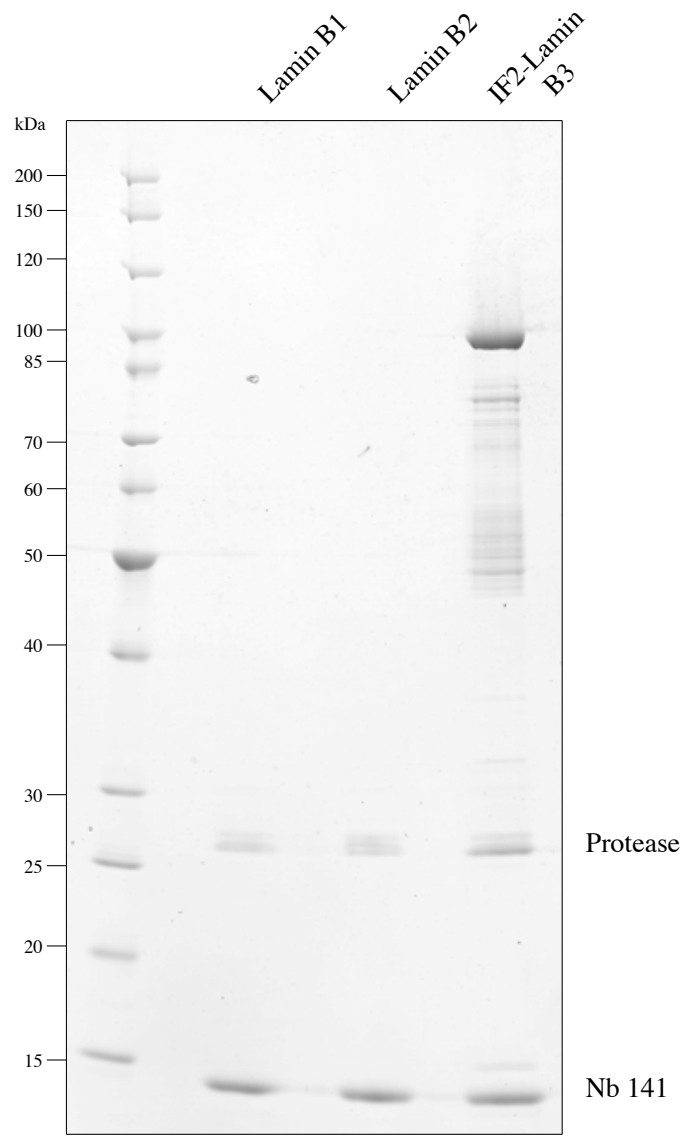

Figure 3.27 Affinity isolation of recombinantly expressed lamin isoforms B1, B2, and B3. Nb152 (left-hand side) binds all recombinantly expressed lamin type B isoforms. In contrary, $\mathrm{Nb} 141$ (right-hand side) binds only lamin B3 isoform and not the other lamin type B isoforms. In short, recombinantly expressed lamin isoforms were incubated with pre-immobilized nanobodies. Formed complexes were protease eluted and mixed with SDS loading buffer. Samples were analyzed on SDS-PAGE followed by Coomassie staining.

Affinity isolation of lamin type-B isoforms shows that the nanobodies $\mathrm{Nb} 141$ and $\mathrm{Nb} 152$, although orthogonal to each other, show different binding preferences towards lamin $\mathrm{B}$ isoforms. Nanobody Nb152 exhibits the ability to bind all lamin B forms from X. laevis. In contrast, Nb141 is specific for lamin B3 isoform and gives no detectable signal when incubated with B1 or B2 lamins. This finding appeared somehow unexpectedly, given that both orthogonal anti-lamin antibodies are targeting the very same 33-215 amino acid region of lamin B3. However, when scrutinizing the sequence of lamin B1 and B2 isoforms one can notice that they have quite a high degree of sequence similarities in the region 33-114 when compared to lamin B3. A logical assumption could be made that 33114 amino acid is the region where the nanobody Nb152 binds. On the other hand, the 115-215 amino acid region has more differences among the lamin B isoforms, which could give a hint as to where the Nb141 might bind, as it is lamin B3 isoform specific. The 
finding that the nanobody 152 is able to bind all the lamin B isoforms from X. laevis allows its application in other cell types, and not only in oocytes, where lamin B3 isoform is predominant. On the other hand, molecular tools that specifically recognize lamin B3 could be utilized in in vitro assembled nuclei assays, where B3 is a major lamin isoform.

\subsubsection{Native isolation of lamin isoforms from Xenopus laevis eggs}

Affinity chromatography is one of the most powerful method to isolate proteins of interest. Oftentimes this method has also no alternatives when protein purification under physiological conditions is desired. After confirming that our newly developed anti-lamin nanobodies are capable to capture recommbinantly expressed lamin isoforms we set out to establish an experimental system to test if the isolation of native lamins is possible.

Lamin B3 is reported to be expressed in some somatic tissues e. g. neurons, muscle cells, but the strongest expression of lamin B3 being in the oocytes and eggs (Benavente et al., 1985). These cells are also easily amenable to in vitro experiments and can be isolated in high homogeneity and quantity. Thus, we have prepared $X$. laevis egg extract as described previously (Blow and Laskey, 1986) and used it as starting material for the affinity isolation of lamin B3.

Lamin exists in two forms in the cell: as a monomer (Gerace and Blobel, 1980) or as a polymer of a defined structural order (Aebi et al., 1986). The lamin B3 monomers have substantially higher level of phosphorylation compared to the polymers. Previous work has shown that okadaic acid is a potent phosphatase inhibitor. Okadaic acid inhibits glycogendependent lamin polymerization (Lourim and Krohne, 1998). Lourim et al. concluded that okadaic acid inhibits the function of a protein phosphatase PP1 that has a direct impact on lamin polymerization. Based on these evidences we assumed that the addition of okadaic acid might prevent lamin B3 polymerization, thus allowing a better accessibility of lamins by the nanobodies. We prepared the egg extract as described by Laurim et. al, in the presence of $2 \mu \mathrm{M}$ okadaic acid. Nanobodies Nb141 and Nb152 were pre-immobilized onto streptavidin magnetic beads. Next, the okadaic acid-treated egg extract was incubated with the nanobodies for 45 minutes, washed, and eluted with the SUMO protease, granting a mild elution of the complex. We could detect no lamin B3 eluted from the okadaic acid treated sample. Lamin B3 upon dephosphorylation becomes monomeric, however, seems to be still bound to membranes (Gerace and Blobel, 1980). 
As all lamins type-B are believed to be permanently isoprenylated and likely to be membrane anchored, we decided to test if the nanobodies could bind lamin in its polymeric form. Lamin is polymerized in the nucleus, however, the association with the membranes might adversely affect the accessibility and consequently the purification of the lamina. It was reported previously that Triton X100 treatment does not disrupt the high order organization of the lamina, on the other hand, as a detergent, Triton X100 effectively solubilizes the membranes (Lehner et al., 1986).

To achieve the desired goal we have added $0.25 \%$ Triton X100 directly to the egg extract and incubated it for 45 minutes. Afterwards, the treated egg extract was incubated with the pre-immobilized nanobodies $\mathrm{Nb141}$ and $\mathrm{Nb152}$. To detach the nanobodies from the matrix they were eluted with the SUMO protease. The affinity chromatography demonstrates the enrichment of lamin B3 on the Nb141 and Nb152 matrix Figure 3.28.

The isolated complexes were analyzed by mass spectrometry confirming the lamin B3 presence. Interestingly, together with the lamin B3 there is also a considerable amount of lamin B4, which migrates approximately at $71 \mathrm{kDa}$. This is rather a surprising finding, as lamin B4 was reported to be expressed only in X. laevis post meiotic male germ cells and when the egg extract was analyzed no lamin B4 was detected (von Moeller et al., 2010). However, von Moeller et al. analyzed only lamin B4 mRNA levels and not protein levels. Thus, the discrepancy in the results might be explained by different methods used. 
Lamin isolation from $X$. laevis egg extract treated with Triton $\mathrm{X} 100$

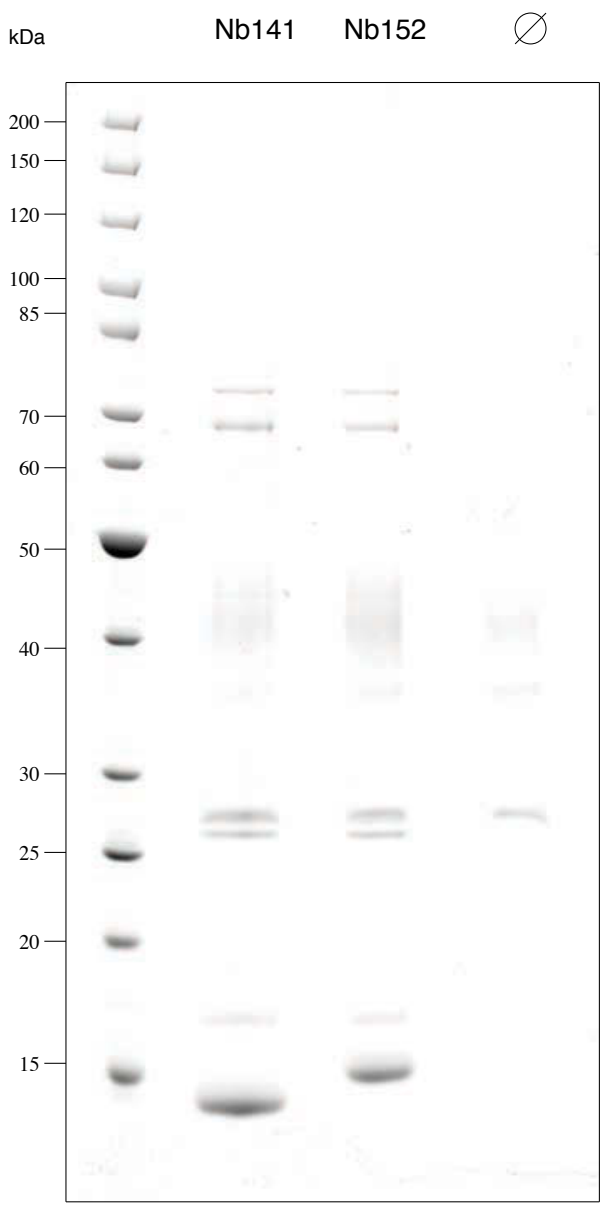

Lamin isolation from $X$. laevis egg extract treated with Okadeic acid

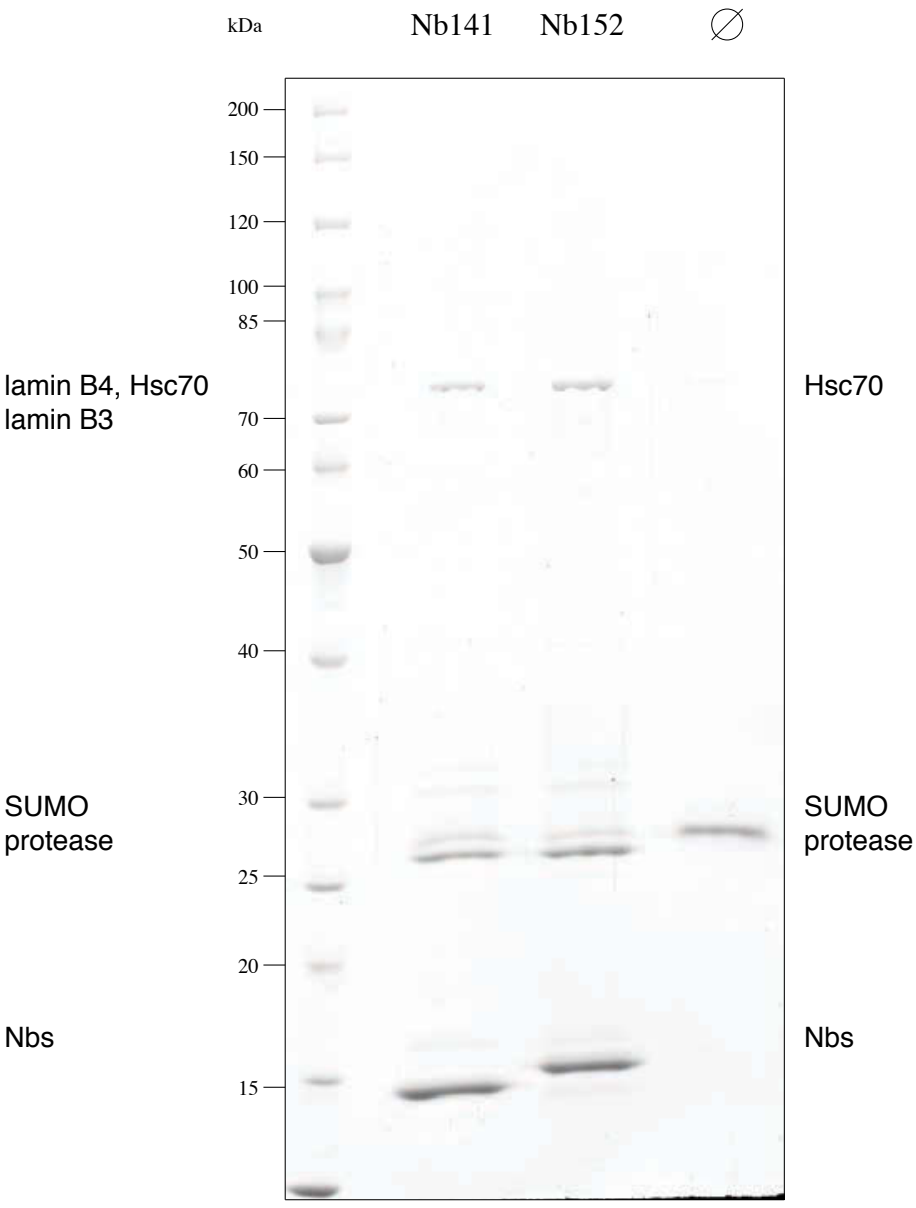

Figure 3.28 Native lamin isolation from $X$. laevis egg extract. Affinity capture of lamins via nanobodies Nb141 and Nb152 in presence of Triton X100 and Okadaic acid. Mass spectrometry analysis confirmed that the respective bands are lamin B3 and lamin B4, left hand side picture. In addition, Nb152 was also positive in isolating lamin B1 and B2 as detected by mass spectrometry results. No lamin was detected upon the treatment of the egg extract with $3 \mu \mathrm{M}$ Okadaic acid. The molecular weight of the isolated lamin isoforms closely matches predicted theoretical molecular weights of the proteins. Lamin isoforms were isolated from $X$. laevis eggs in presence of $0,25 \%$ Triton X100. Isolated Nb/lamin complexes were eluted by SUMO protease and mixed with SDS loading buffer. Next, the samples were analyzed on an SDS PAGE gel and stained with Coomassie. Mass spectrometry analysis was performed in Prof. Urlaub laboratory by Monika Raabe.

Additionally, mass spectrometry analysis of the Nb152 eluate shows the presence of lamin $\mathrm{B} 1$ and B2 isoforms. This result supports the previous finding that the Nb152 is a not lamin B3 specific and binds all type B lamins. In contrast, there was no lamin B1 or B2 detected in the eluate of nanobody 141.

The eluate of $\mathrm{Nb} 152$ and $\mathrm{Nb} 141$ was analyzed by mass spectrometry and the relative abundance of lamin isoforms was analyzed by iBAQ Figure 3.29. Relative protein abundances of isolated lamin isoforms correlate well with previously published total 
protein concentrations in Xenopus laevis eggs (Wühr et al., 2014). It is worth mentioning that lamin B4 is partially masked by Hsc70, which co-migrates with it.

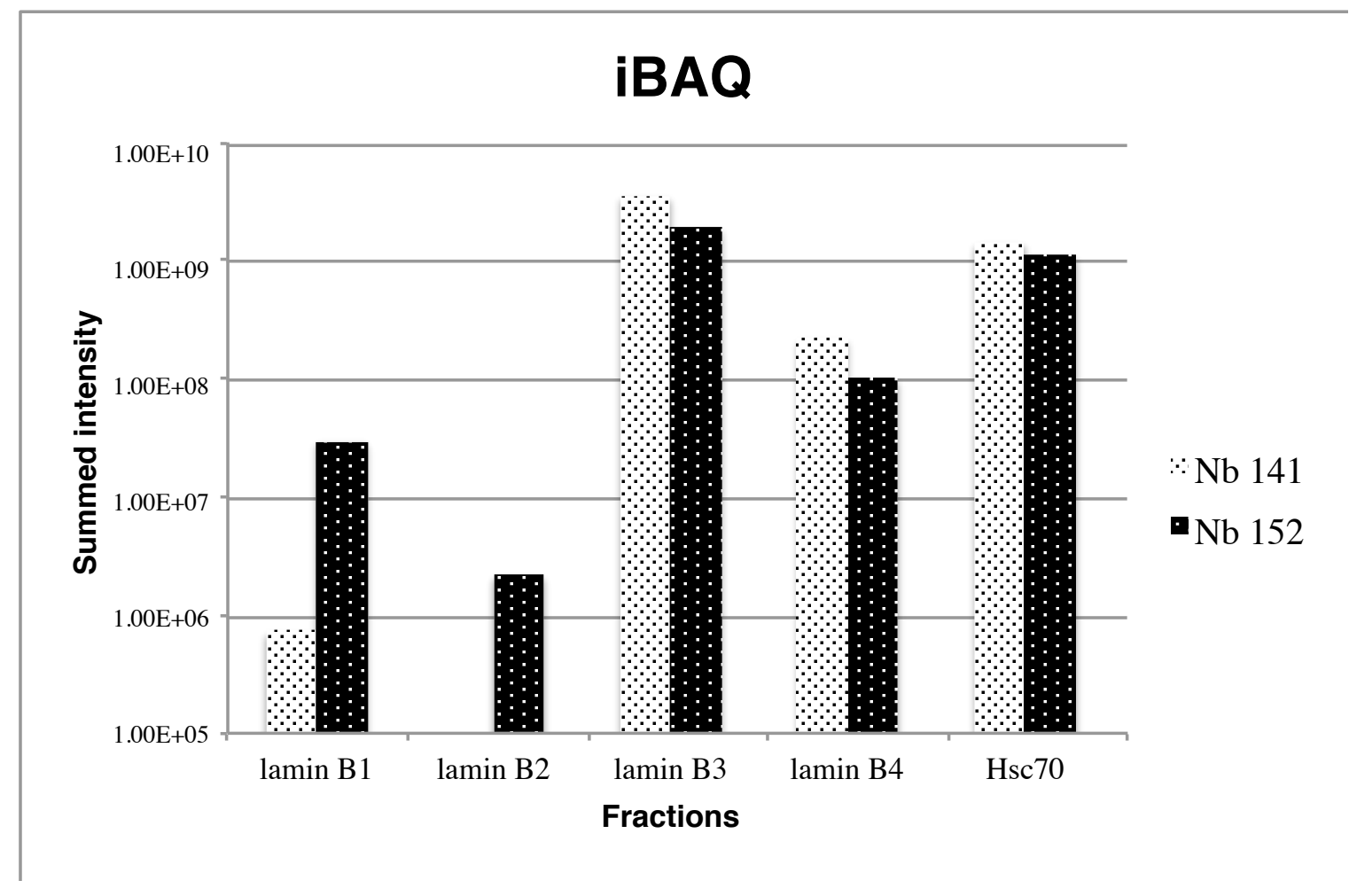

Figure 3.29 Comparison of label-free quantification (iBAQ) of lamin isoforms isolated by nanobody Nb152 and Nb141 from $X$. laevis egg extract (Figure 3.28). The sum of all laminisoform identified peptide intensities was divided by the number of theoretically observable peptides. Note the differences in relative protein quantities of lamin B1 and lamin B2 in samples isolated by $\mathrm{Nb} 141$ and $\mathrm{Nb} 152$. No differences are observed in lamin B3 quantities among Nb141 and $\mathrm{Nb} 152$ isolation. The mass spectrometry analysis was performed by Monika Raabe in the laboratory of Prof. Urlaub.

Several conclusions can be drawn from the native lamin affinity purification. Firstly, both nanobodies are able to bind lamin under native conditions. Secondly, the spectrum of lamins that can be found differs among the two nanobodies. Last but not least, while Nb141 can be used to immobilize B3 and B4 isoforms the Nb152 binds all lamin B type lamin isoforms found in X. laevis egg extract.

\subsubsection{In vitro nuclei assembly investigation by anti-lamin nanobodies}

Since it was fist described, the in vitro nuclei reconstitution method has become one of the major techniques to study numerous nuclear components and their functions (Forbes et al., 1983; Lohka and Masui, 1983). Depletion of proteins by antibodies, addition of endogenous engineered protein mutants, or fully exogenous proteins, to name a few, are 
approaches commonly used to study nuclear structures or their function. These methodological advancements have also fostered the understanding of the nuclear lamina as an important structural component of the nucleus (Goldberg et al., 2008).

Antibodies are necessary tools to perform numerous manipulations on in vitro nuclei assembly. Antibodies are used not only to deplete molecules of interest, but also to visualize nuclear components during or after the nuclear assembly takes place (Dudek et al., 2010).

We wanted to test if our selected nanobodies can be used as molecular markers to visualize lamina by the means of in vivo nuclei assembly. To achieve this goal nanobodies were fluorescently labeled via engineered cysteines (Pleiner et al., 2015). After achieving a complete nanobody labeling, $\mathrm{Nb} 141$ and $\mathrm{Nb} 152$ were used to label in vitro assembled nuclear lamina Figure 3.30.

A.

Assambled nuclei stained with anti Lamin B3 nanobodies

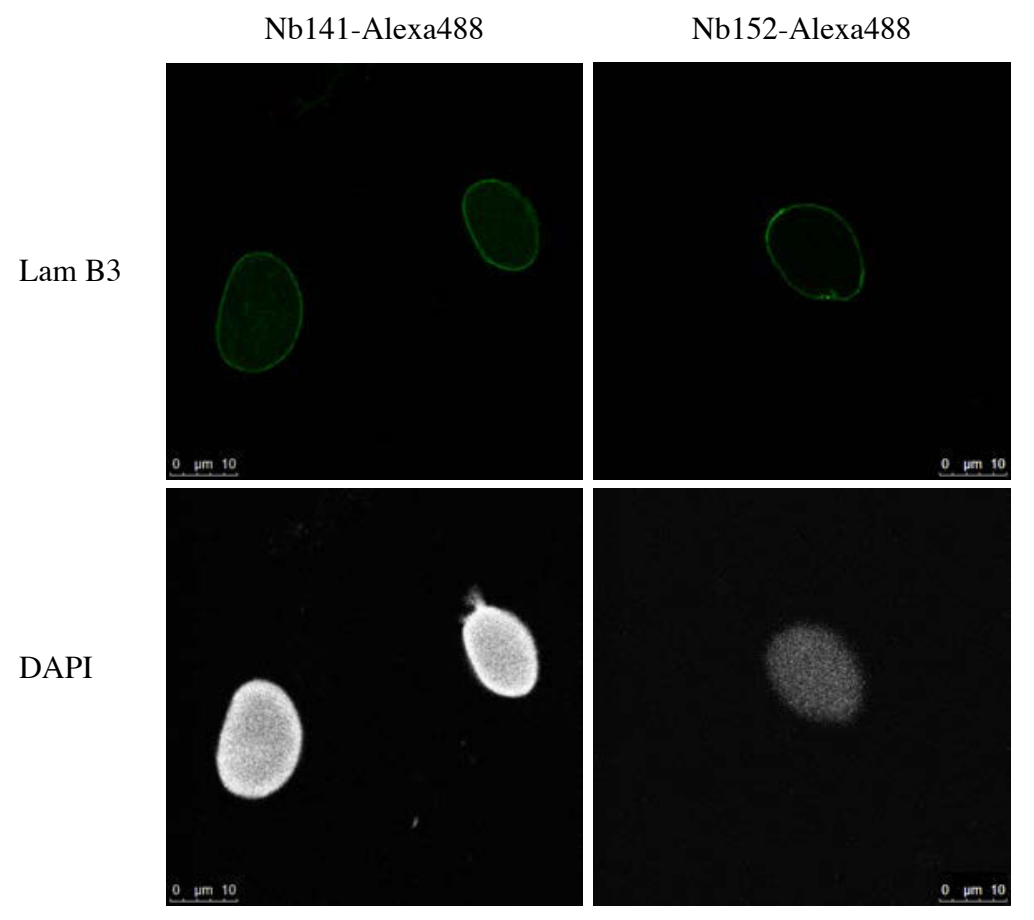




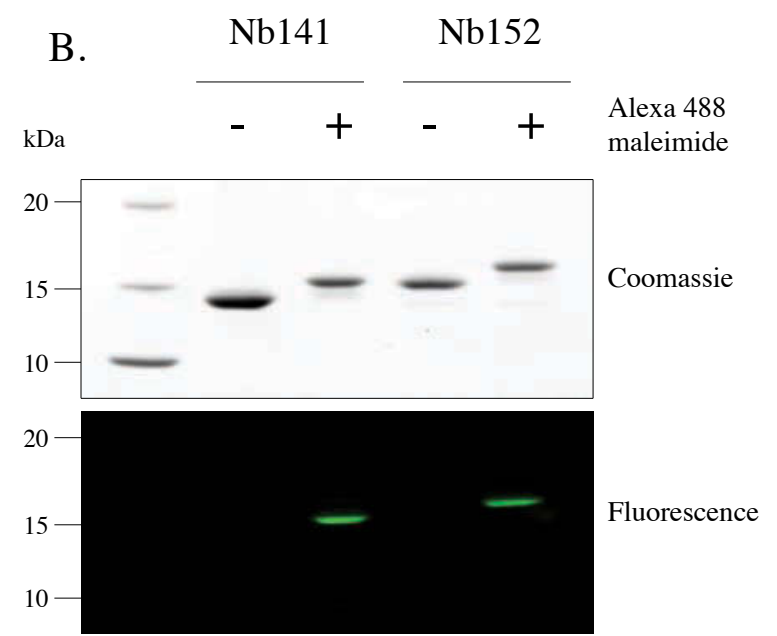

Figure 3.30 Immunofluorescence with sitespecifically labeled nanobodies. In vitro assembled nuclei were assembled, fixed with $2.6 \%$ paraformaldehyde, permeabilized with $0.3 \%$ Triton $\mathrm{X} 100$, and stained with $\mathrm{Nb} 141$ and $\mathrm{Nb} 152$. Note the characteristic nuclear rim staining of lamina (A) The concentration of the nanobodies applied to the assembled nuclei is 2 nM. The nanobodies Nb141 and Nb152 were fluorescently labeled with Alexa488 via an engineered C-terminal cysteine (B). Dapi is depicted in gray, nanobodies are in green.

We have further tested if anti-lamin B3 nanobodies have an effect on in vivo lamin assembly. To test this, we have added one nanobody directly to the assembly reaction. At the end of the assembly the nuclei were fixed and stained with the orthogonal anti-lamin nanobody partner (Nb141 for Nb152 and vice-versa). As can be observed in the Figure 3.31 the lamina of assembled nuclei contains fiber-like constituents. This phenotype may indicate that the addition of nanobodies captures or freezes the lamina in an intermediate form during its assembly.

Nuclei assambled in presence of anti Lamin B3 nanobodies

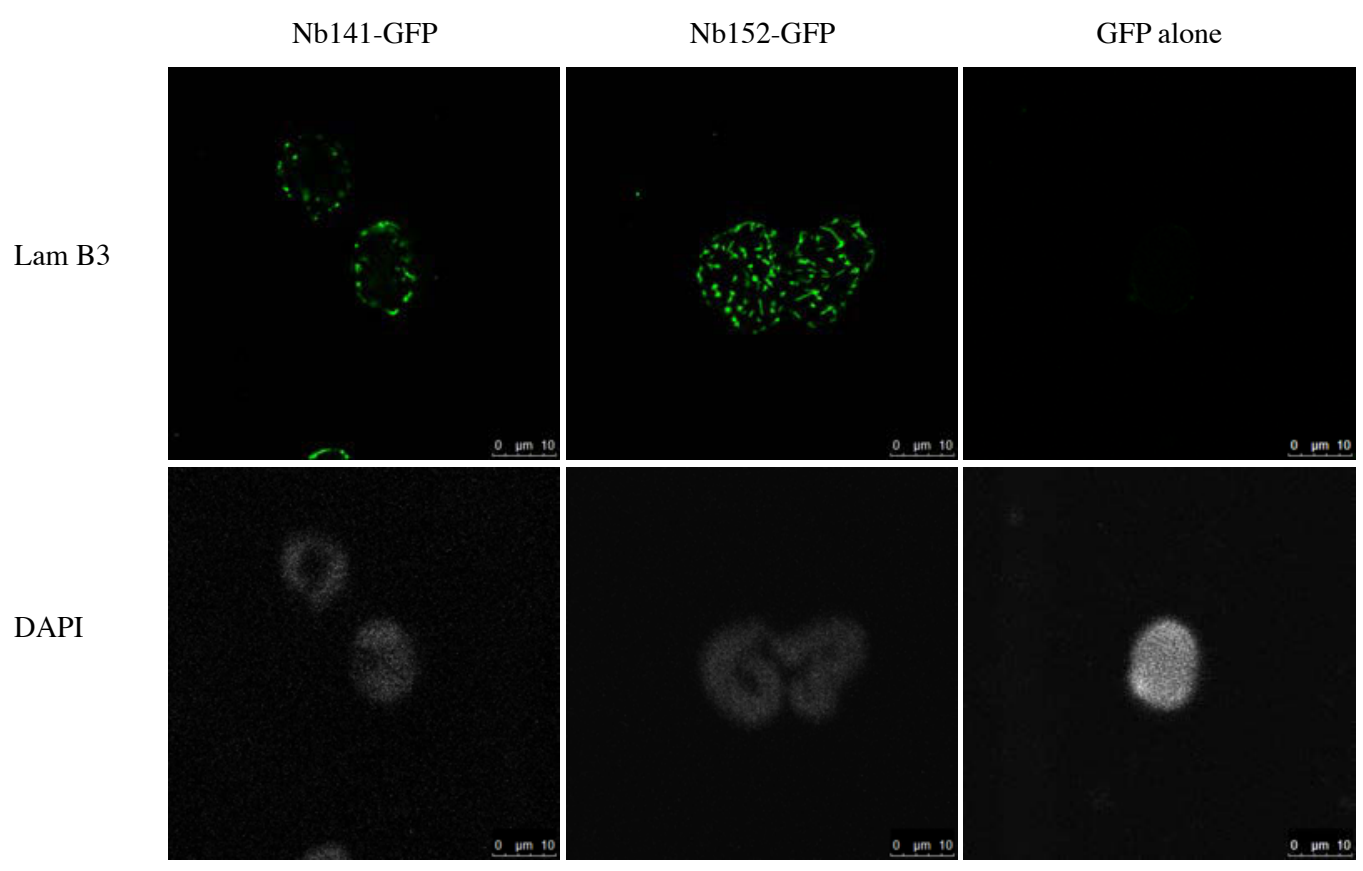

Figure 3.31 Influence of anti-lamin B3 nanobodies on the lamina assembly. Nb141 and Nb152 were added during the nuclei assembly reaction in $150 \mathrm{nM}$ concentration. In vitro nuclei were assembled, fixed with $2.6 \%$ paraformaldehyde, permeabilized with $0.3 \%$ Triton X100, and stained with Nb141-GFP or Nb152-GFP. Dapi is depicted in gray, nanobodies are in green. 
Next, we wanted to test if this effect is dependent on concentration of nanobodies. For that, we assembled nuclei in the presence of varying concentrations of nanobodies Figure 3.32.

$\mathrm{Nb}$ added / $\mathrm{Nb}$ used for staining

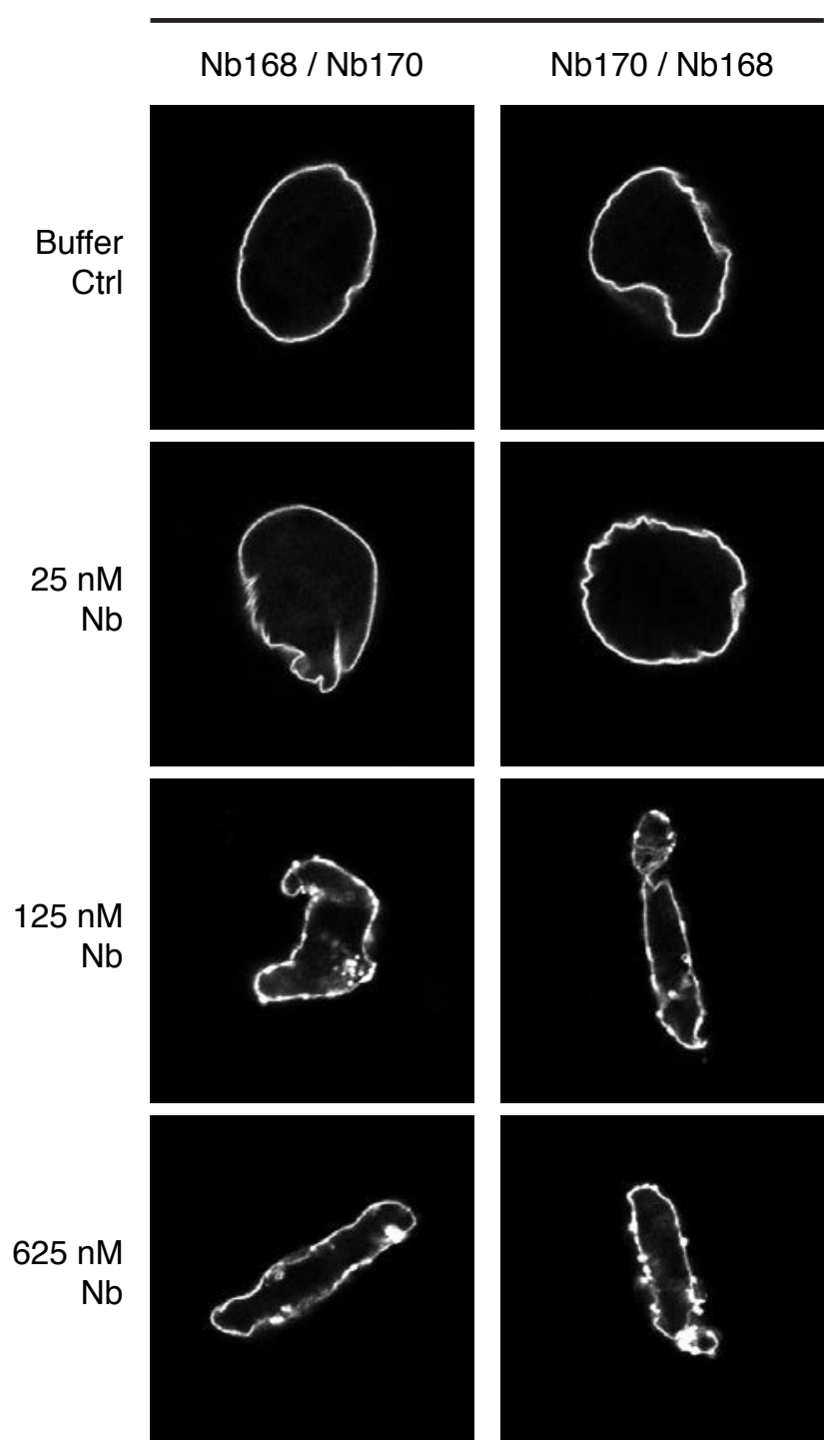

Figure 3.32 Nuclei assembled in presence of fluorescently labeled Nb141 or Nb152. Non-labeled Nb141 or Nb152 were directly added to the assembly mixture and the reaction was initiated by the addition of the sperm chromatin. Upon their assembly the nuclei were fixed and stained with the orthogonal nanobody. Different concentrations of non-labeled nanobodies were tested, while the Alexa488 labeled nanobody concentration was kept constant at $20 \mathrm{nM}$. Note that he nuclei were imaged at the middle point. Dr. Bastian Huelsmann kindly performed the nuclei assembly reaction and imaging.

Non-labeled nanobodies were added during the nuclei assembly reaction in ascending concentrations. The nuclei assembled in presence of an anti-lamin nanobody in concentrations as low as $100 \mathrm{nM}$ are smaller compared to the control, as well as the shape of the nuclei is disturbed. 


\section{Discussion}

\subsection{SUMO protease cleavable phage M13}

During the last decades there were numerous technological advancements in the field of protein display. In general, there are several conceptually different techniques to display a protein, which are now available to researchers. All display techniques could be divided into several groups: display on cells (yeast, bacterial, or mammalian cells), protein-mRNA displays (ribosome and mRNA display), display on micro beads, and finally, display on viral particles. All of the mentioned display techniques have four key features in common: production of genotypic diversity and its cloning, coupling of genotype and phenotype, applicability and withstanding of the selective pressure, and lastly, amplification of the selected genetic material.

Without a doubt the phage M13 has become one the mainstream platforms for antibody or nanobody selection and engineering. Phage display on M13 is time-proven to be a robust technique for construction of libraries with millions or even billions of different proteins, at the same time, the method is also versatile and simple to use. Bacteriophage M13 incurs its status due to its stability under various conditions, including $\mathrm{pH}$, different salt concentrations, temperatures, and detergents (Branston et al. 2013). Also, M13 allows a custom design of antibody selection protocols. The desired characteristics of a displayed protein are selected via panning and a resilient displaying platform is of a crucial importance.

As a commonly utilized tool the phage M13 was highly optimized and modified to the needs of research. However, the "Achilles heel" of the phage M13 is its elution, i. e. the recovery of phages in the bio-panning. The goal of elution is to dissociate the displayed protein from the antigen, but it is not feasible to establish specific conditions for a desirable mild elution for every antigen. Thus, the most common approach is to subject enriched phages to harsh conditions, at which they dissociate from the antigens. An exemplary elution method is a $\mathrm{pH}$ elution using very acidic $\mathrm{pH} 2.2$ or very basic $\mathrm{pH} 12-13$ buffers. Although generally used, experimental data suggests that M13 viral particles are stable in the $\mathrm{pH}$ range between 3 and 11, where $\mathrm{pH}$ values outside of this range affect the M13 phage viability drastically (Branston et al., 2013). Also, very affine binders could be simply resistant to the $\mathrm{pH}$ treatment and as a result not eluted. An even more negative 
effect associated with the $\mathrm{pH}$ elution is that, undesirable, non-specifically bound phages are also eluted under the $\mathrm{pH}$ elution conditions.

In this study we present a new advanced phage elution method based on the SUMOcleavable M13 phage. We have introduced a SUMO protease cleavable module between a displayed protein and the pIII coat protein of M13 phage. The SUMO module has several advantages over other methods of phage elution. First of all, it allows elution of phages that are specifically bound to an immobilized target. If phages are stuck non-specifically to the selection media e. g. tube plastic, matrix etc. they will be virtually not present in the eluate. This, in turn, leads to the key advantage of the specific phage elution: the higher the elution specificity of selected phages, the higher the chances are to select a desired protein. A protease phage elution is, undoubtedly, the most specific method to elute phages. Here it must be mentioned, that the development of trypsin-cleavable M13 phages was reported (Patent number EP1950295 A1). Although used, these phages have several disadvantageous in comparison to the SUMO cleavable M13 phage. Trypsin protease recognizes a single amino acid: arginine or lysine. This can impose a risk to cleave also viral proteins, thus compromising the phage infectivity. The SUMO protease recognizes a protein fold, rather then a specific residue and carries no risk for the viral particles.

The time of phage elution should be short, to save valuable hands-on time. Based on the comparison of protease efficiencies, SUMO proteases are up to 1000 times more efficient than the TEV protease, thus, SUMO proteases are some of the most efficient proteases (Frey and Görlich, 2014). Using the SUMO-cleavable phage M13 we were able to shorten the elution time to 5 minutes at 37 degrees.

An alternative protease elution method is an introduction of a protease cleavage site directly into the antigen construct, allowing a protease elution of the immobilized antigen (Dr. Tino Pleiner PhD Thesis). However, when a recombinant protein production is not possible, or when panning is done against large protein complexes, a SUMO-cleavable phage becomes an ideal elution tool. Also, in the case of antigen cleavage, the cleaved antigen stays attached to the phage. This can have deleterious effects on the phage infectivity and is prohibiting elution of large antigens. The SUMO-protease cleavable M13 phage is, essentially, antigen free after the elution. When the bulk of a displayed protein is removed, the infectivity of phages is not hindered in any way. This feature is also of an asset, when a polyvalent display on pIII is desired. It is generally known, that a polyvalent 
display on pIII protein of M13 suffers from a compromised infectivity. The pIII protein is a major protein in the process of attaching a phage to a target cell, thus, the size of a displayed protein is limited to 20-30 amino acids. We propose that SUMO-cleavable phages could be successfully implemented for the polyvalent protein display on the M13 phages. Due to the fact that the N-terminus of pIII can be restored in its native form, considerably larger proteins could be displayed with no risk of hindering phage infectivity.

Yet another application of the SUMO-cleavable phage M13, which we could envision, is a SUMO proteases substrate selection. When the most $\mathrm{N}$-terminal protein remains constant and is bound to a matrix, one could display libraries of protease cleavable modules, where the most suitable variants would be eluted first. Thus, by varying the time and the cleavage conditions proteases with new desired characteristics could be obtained.

In nutshell, our SUMO-cleavable M13 phage uses the SUMO protease, which enables a novel, efficient method of phage elution. Displayed proteins can be specifically eluted under mild physiological conditions with no harm to the phage itself. Additionally, due to the high SUMO protease activity a very short elution time is required, where virtually all the phages can be eluted.

\subsection{Nanobodies against Tth ribosomes and the rRNA binding nanobody}

In order to identify the number of $\mathrm{Nb} / \mathrm{NTRs}$, sufficient for the transport of a ribosomal subunit, several technical improvements in the preparation of ribosomes and bio-selection of nanoboides had to be made. First of, we have purified ribosomal subunits from Thermus thermophilus to a high homogeneity. The ribosomes were successfully used for immunization of alpacas and construction of highly complex nanobody libraries. In silico, we have identified the cysteines on the surface of ribosomes and experimentally established that they can be efficiently biotinylated with maleimide-PEG11-biotin. The biotinylation of ribosomes, in turn, allowed a simple and specific immobilization of ribosomal subunits onto streptavidin magnetic beads. Additionally, the long PEG spacer arm adds length and reduces steric hindrance of immobilized ribosomes, which accounts for accessibility of nanobodies to all the ribosomal proteins.

Next, complex nanobody libraries were displayed on our newly developed SUMOcleavable M13 phage. The SUMO-cleavable phage M13 was successfully used to elute phages and due to its high specificity the majority of eluted phages are positive binders of 
the ribosomes. In total, we generated six orthogonal anti-Tth ribosome nanobodies. All the nanobodies can be used for affinity capture and purification of ribosomes from the Tth crude lysate, due to the their high affinity and specificity. The nanobody $\mathrm{Nb} 48$ deserves, however, a special attention. We established that this nanobody targets L7/12, a protein, which is present in six copies per ribosome in T. thermophilus. Although the Tth ribosomes were crystallized, the L7/12 is disordered and absent in the structure of 50S. Due to a high degree of molecular mobility the L7/12 it cannot be reliably interpreted, even when present on $50 \mathrm{~S}$. We propose that our nanobody $\mathrm{Nb} 48$ could be used either as a crystallization chaperone of $\mathrm{L} 7 / 12$ or it could offer means to stabilize the $\mathrm{L} 7 / 12$ as a part of the ribosome.

We also would like to report the very first nanobody against nucleic acid. Our CRAC experiments (in collaboration with Prof. Bohnsack) show that the Nb50 is a true binder of the rRNA and it binds an A-site of the 50S subunit. Although there were articles reporting selection of anti-nucleic acid antibodies, they are still uncommon, in the nanobody research field there are no reports on nanobodies, which could carry a similar function. One explanation for the absence of any anti-nucleic acid nanobodies could be that nanobodies preferably bind to non-liner antigens. Ribosomal RNA, however, is probably an ideal antigen, in order to select a nucleic acid binding nanobody. The reason being that the rRNA is a conformationally stable entity, which can in fact carry out its ribozyme functions even without ribosomal proteins (Noller et al., 1992).

By this example, we show that nanobodies could be also selected against nucleic acids, provided that they have conformationally stable secondary structures.

\subsection{Mapping of nanobodies binding sites on large antigens}

A number of methods is commonly used to characterize binding sites of antibodies. These include cryo-EM, X-ray crystallography, antigen fragmentation, cross-linking coupled to mass spectrometry analysis. The first two methods are, undoubtedly, the golden standard in characterization of antibodies, providing most of the information about antigen-antibody interactions. Despite of their obvious advantages, crystallography and cryo-EM are time and labor intense and cannot be used for every antibody/antigen characterization.

We have successfully used the antigen fragmentation method to establish binding domains of anti-lamin B3 nanobodies. The prerequisite of the given method is the recombinant antigen expression. This imposes several limitations: first, not every antigen can be 
expressed recombinantly, and secondly, it is, sometimes, difficult to establish the boundaries of protein domains for their correct expression. This approach is preferable for the characterization of antibodies, as they, oftentimes, recognize linear epitopes. Nanobodies, however, prefer to bind complex conformational epitopes, which might not be preserved when protein truncations are made.

For the analysis of more complex samples e. g. large complexes a more advantageous method is protein cross-linking followed by mass spectrometry analysis. In this work we have successfully employed this method for the identification of antigen of ten antiribosomal nanobodies. An introduction of an affinity tag onto nanobodies helps to circumvent a common problem associated with the sample enrichment. A simple His-tag allowed us to capture and enrich the cross-linked material on the $\mathrm{Ni}^{2+}$ matrix. We show that a series of SDS washes can be applied to remove virtually all the ribosomal proteins including rRNA, to further reduce the sample complexity. Finally, an imidazole SDS containing buffer yielded an excellent elution media and samples could be directly used for mass spectrometry analysis.

\subsection{Nanobodies as tools to study transport of ribosomal subunits}

To test if the anti-ribosomal nanobodies could be used as a platform to recruit the NTRs we have fused them to NES or NLS sequences. Nb-NES fusions turned to be proteolytically cleaved, while expressed in E. coli. This technical obstacle, however, can be overcome by performing a double tag purification. The $\mathrm{Nb}$-importin $\beta$ fusions are well expressed with no degradation products. When the ribosome/ $\mathrm{Nb}$-importin $\beta$ complexes were formed and used in FG-particles assay, the importin $\beta$ alone could bind ribosomal proteins even with no $\mathrm{Nb}$ present. This, somehow unexpected, technical problem could be explained by ribosomal proteins dissociating from the ribosome and then being bound by the importin $\beta$, or that importin $\beta$ has an affinity to bind basic ribosomal proteins directly even with no NLS present on them. In both cases more experimental data are needed to clarify this result. 


\subsection{Recombinant expression of soluble lamins}

Lamins are intermediate filaments and, as such, they form polymers under physiological conditions. Since their discovery it was observed that lamins form inclusion bodies when expressed in E. coli. This observation could be explained by the fact that the cytoplasm of E. coli is of $\mathrm{pH}$ values from 7.2 to 7.8 and at exactly these $\mathrm{pH}$ conditions lamins polymerize (Karabinos et al., 2003). Recombinant expression and isolation of proteins, nevertheless, is a pivotal step for numerous biochemical assays, let alone the immunization of animals for antibody productions.

Since several decades, the purification of lamin from E. coli inclusion bodies was the method to use, when recombinant lamin was needed. Undoubtedly, this method is laborintense, time consuming and could not be called physiological. Refolding of proteins from inclusion bodies is not only a cumbersome process, it also requires testing numerous conditions for a mild protein solubilization and, even when optimized, results in a poor protein recovery.

We would like to report a purification strategy to obtain recombinant lamins in a soluble form and this, to our best knowledge, is the first time such a strategy is reported. In this work we have tested and established methods for lamin purification from E. coli using a couple of different solubility tags. A common strategy to solubilize aggregation prone proteins is their fusion to a solubility enhancing partner. Surprisingly, there are no published articles presenting lamin solubility tags. We have chosen a couple of commonly used solubility tags and tested their effects on lamin. First, we have tested a commonly used IF2 tag (Sørensen et al., 2003). The expression of lamin with IF2 tag rendered almost completely soluble lamin. It is worth to note, however, that when a SUMO protease cleavage site was introduced into the construct, it was partially degraded inside E. coli cells. Nevertheless, it seems that the IF2 tag does not hinder the polymerization propensity of lamin B3 protein, as we observed by the negative stain analysis.

In order to obtain lamins in a soluble tag free form we have also tested lamin-MBP fusions. Using this strategy we could successfully purify all three lamin type-B isoforms from $X$. laevis in a tag-free form, as the addition of SUMO-cleavable module was not causing any cleavage in the E. coli cells. Also, the yields of lamin purification with these tag systems were up to $10 \mathrm{mg}$ per liter of culture. 
In nutshell, we have implemented lamin purification strategy that can be used to prepare considerable amounts of lamin in a tagged or a tag-free form. It offers purification of soluble lamins in a more cost and time effective manner, which, in turn, should facilitate lamin crystallization attempts, and foster biochemical characterization of lamin isoforms, as well as its interacting partners.

\subsection{Anti-lamin B3 nanobodies and their applications}

Xenopus laevis oocyte is one of the few commonly used systems to study lamina organization. Despite of numerous attempts to study the organization of lamina in X. laevis oocyte, there is still an ongoing debate as to how lamina is spatially organized (Goldberg et al., 2008; Turgay et al., 2017). Lamin B3 is a major lamin isoform in X. laevis oocytes and eggs, however, the tools to study lamins are still limiting, both for immunofluorescence, electron microscopy and for immunoprecipitation assays.

In this work we present a set of orthogonal anti-lamin B3 nanobodies, which are a valuable addition to a toolbox to study lamina. Due to our advances in the recombinant soluble expression of lamin we could efficiently produce necessary amounts of recombinant lamin for both immunization and bio-panning. Our newly developed nanobodies are suitable tools for studies of lamin properties by native purification of lamina. Our experiments on lamin extraction, using non-ionic detergents, suggest that the nanobodies isolated lamina, likely comes from already assembled lamina. Such an affinity enrichment on nanobodies can be used to analyze gross post-translational lamin modifications in X. laevis oocytes or eggs, as the phosphorylation status of lamins is of a great interest and it influences the polymerization of lamina. Furthermore, the affinity enrichment of lamin via nanobodies under physiological conditions could facilitate biochemical studies of lamin properties.

There are four lamins type-B in X. laevis: B1, B2, B3, and B4. Lamin B4 is reported to be a germ cell line specific and is not found outside of the germ cell lineges as reported by von Moeller et al., 2010. They suggest that lamin B4 is expressed exclusisvely in testes and sperm of $X$. laevis, with no detectable presence in oocytes or eggs. We, however, found that lamin B4 is also present in the egg extract. Our orthogonal anti-lamin nanobodies were able to enrich lamin B4 in the immunoprecipitation experiments. This discrepancy in obtained results likely comes from different methodologies used to test for lamin B4 presence in the tissues. Von Moeller et al. have tested for the presence of mRNA of lamin B4 in different tissues and not for protein levels of lamin B4. We show the lamin 
B4 presence by means of direct affinity binding and conclude that lamin B4 is present in the eggs of $X$. laevis, together with the other previously reported three lamin type-B isoforms.

The addition of anti-lamin nanobodies in concentrations higher than $100 \mathrm{nM}$ during the $X$. laevis nuclei assembly influences the lamina assembly. The addition of nanobodies during the assembly of nuclei causes a filamentous-like appearance of lamina. This observation might be explained by the assumption that the nanobodis bind lamins at a certain point during the intermediate filament formation, thus creating a hindrance for further proper polymerization. We envision a possible application of the nanobodies for the enrichment and analysis of the lamin B3 intermediates by electron microscopy. Previous studies on the lamina assembly show the in vitro steps of the filaments formation (Aebi et al., 1986; Karabinos et al., 2003). With the help of our newly developed nanobodies a conceptually new method to analyze the intermediates is available, allowing lamina analysis from the egg extract directly, using the wild type lamin B3.

Another application of the anti-lamin B3 nanobodies could be to selectively target lamins to a specific compartment or, conversely, tether proteins of interest to the lamina, thus studying their influence on the lamina. An exemplary set of targets could be protein phosphokinases. Phosphorylation of lamins has been long known to cause lamina disassembly. Viruses, for example, which assemble in the nucleus have to overcome the lamina, which creates a barrier for the transport of the capsids. For instance, viruses from the herpes family utilize a set of phosphokinases to disassemble lamina locally. We anticipate that our newly developed nanobodies could be used to selectively target viral phosphokinases to the assembled lamina and test their influence on its assembly.

Next, the anti-lamin nanobodies could be used to aid lamin crystallization trials. Because lamin is a fibrillar protein, with a great percentage of flexible linkers in its structure, lamin crystallization was exceedingly difficult. A common approach to circumvent this complication was a "divide and conquer" strategy. The structure of the IgG domain (DhePaganon et al., 2002) and a 74 amino acid stretch of the 2B rod domain (Strelkov et al., 2004) are two examples of the studies employing this strategy. Previous positive application for crystallography of protein complexes (Chug et al., 2014) allows us to speculate that our anti-lamin B3 nanobodies could be also used as crystallization chaperons in lamin crystallization trials. 
To shed light onto lamin orientation within lamina a reliable imaging marker is of a great value. Previously, several different methods to visualize lamina were used. For electron microscopy based detection, an oocyte injection of DNA of lamins with a FLAG tag was used (Goldberg et al., 2008). Then, anti-FLAG tag antibodies conjugated to gold particles were used to visualize the lamina meshwork. This method is tedious and the FLAG-tag on lamin could potentially cause incorrect lamina assembly. Most importantly, however, due to their large size, antibodies produce a great misplacement of golden particles from the actual lamin molecules. The nanobodies, on the contrary, are far more superior tools to study organization of tightly assembled molecules. Here, we show that our nanobodies perform well in conventional confocal microscopy to visualize lamin B3. Based on this result, we propose that the anti-lamin B3 nanobodies could be also used to study the lamina organization with super-resolution or electron microscopy. Given that the antilamin nanobodies are orthogonal they could be utilized to map the meshwork of lamin with a great precision.

In sum, we conclude that anti-lamin B3 antibodies are a valuable addition to the tools available for visualizing lamina, as well as studying its biochemical properties. 


\section{Materials and methods}

\subsection{Materials}

\subsubsection{Chemicals}

All general laboratory reagents were obtained from the following manufacturers Calbiochem (San Diego, USA), GibcoBRL-Life Technologies (Paisley, UK), Merck (Darmstadt, Germany), MoBiTech (Göttingen, Germany), Pharmacia (Uppsala, Sweden), Promega (Madison, WI, USA), Qiagene (Hilden, Germany), Roche (Mannheim, Germany), Roth (Karlsruhe, Germany), Sigma-Aldrich (St. Louis, USA), Tosoh (Tokyo, Japan), New England Biolabs (USA), Jena Bioscience (Jena, Germany).

\subsubsection{Instruments and laboratory equipment}

Table 5.1 Laboratory equipment

\begin{tabular}{|l|l|}
\hline Instrument & Manufacturer \\
\hline GenePulserTM & BIORAD, Burlington, USA \\
\hline Eppendorf biophotometer & Eppendorf, Germany \\
\hline Sonifier 450 & Branson, UK \\
\hline UV-table & Benda Laborgeräte, Germany \\
\hline Perfection V700 Photo Scanner & Epson, Japan \\
\hline Incubator/Climo-shaker ISF1-X & Kuehner Shaker, Switzerland \\
\hline Thermomixer comfort & Eppendorf, Germany \\
\hline Thermo NanoDrop 2000C & PeqLab, Germany \\
\hline SensoQuest lab-cycler & SensoQuest, Göttingen \\
\hline Äkta-Explorer, Äkta-Purifier & Pharmacia, Uppsala, Sweden \\
\hline Leica MZ6 microscope & Leica, Mannheim, Germany \\
\hline Monolith NB. 115 & Nano Temper, Germany \\
\hline
\end{tabular}




\begin{tabular}{|l|l|}
\hline Analytical scale & Sartorius, Germany \\
\hline Arium® Pro UV ultrapure water system & Sartorius, Germany \\
\hline Odyssey infrared imaging system & LI-COR Biosciences, USA \\
\hline Cell culture hood Herasafe & Thermo Scientific, USA \\
\hline Eppendorf 5415R and 5424 tabletop centrifuges & Eppendorf, Germany \\
\hline Multifuge 3L-R & Heraeus, Germany \\
\hline Sorval RC6+ centrifuge & Sorvall/Thermo Scientific, USA \\
\hline Sorval Lynx 6000 & Sorvall/Thermo Scientific, USA \\
\hline Sorval Wx Ultra ultracentrifuge & Sorvall/Thermo Scientific, USA \\
\hline Discovery M120 SE ultracentrifuge & Sorvall/Thermo Scientific, USA \\
\hline French press & Avestin, Canada \\
\hline Hi Trap Butyl column, Nap5, PD-10 & GE Gealthcare Life Science, USA \\
\hline
\end{tabular}

Table 5.2 Protein buffers and solutions

\begin{tabular}{|c|c|}
\hline Solution & Composition \\
\hline $\begin{array}{l}\text { 0x PfuS polymerase } \\
\text { buffer }\end{array}$ & $\begin{array}{l}20 \mathrm{mM} \text { Tris- } \mathrm{HCl} \mathrm{pH} \mathrm{9.0,250} \mathrm{mM} \mathrm{KCl,} 15 \mathrm{mM} \mathrm{MgSO}_{4}, 100 \mathrm{mM} \\
\left(\mathrm{NH}_{4}\right)_{2} \mathrm{SO}_{4}, 1 \% \text { Tween } 20,1 \mathrm{mg} / \mathrm{ml} \mathrm{BSA}\end{array}$ \\
\hline 10x Ligation buffer: & $\begin{array}{l}500 \mathrm{mM} \text { Tris pH 7.5, } 100 \mathrm{mM} \mathrm{MgCl}_{2}, 100 \mathrm{mM} \text { DTT, } 10 \mathrm{mM} \text { ATP, } \\
250 \mathrm{~g} / \mathrm{mL} \text { BSA }\end{array}$ \\
\hline DNA ladder & $50 \mathrm{ng} 1 \mathrm{~kb}$-Ladder or $50 \mathrm{ng} 100 \mathrm{~kb}$-Ladder in Orange sample buffer \\
\hline $\begin{array}{l}\text { Orange G DNA } \\
\text { loading buffer }\end{array}$ & $\begin{array}{l}10 \mathrm{mM} \text { Tris }-\mathrm{HCl} \mathrm{pH} \mathrm{8.0,10} \mathrm{mM} \mathrm{EDTA} \mathrm{pH} \mathrm{8.0,50 \%} \mathrm{Glycerol} \mathrm{(w/v),} \\
0.25 \% \text { OrangeG (w/v) }\end{array}$ \\
\hline $50 x$ TAE & $\begin{array}{l}242 \mathrm{~g} \text { Tris Base, } 57.1 \mathrm{~mL} \text { acetic acid, } 100 \mathrm{~mL} 0.5 \mathrm{M} \text { EDTA pH 8.0, } \\
\text { add to } 1 \mathrm{~L} \text { with } \mathrm{ddH}_{2} \mathrm{O}\end{array}$ \\
\hline Tris/HCl pH 7.5 & $\begin{array}{l}\text { Prior to usage, the } \mathrm{pH} \text { was measured and, if necessary, manually } \\
\text { adjusted } \\
16.57 \mathrm{~g} \text { Tris Base and } 136.04 \mathrm{~g} \text { Tris/ } \mathrm{HCl} \text { were diluted in } 1 \mathrm{~L} \mathrm{ddH_{2 } \mathrm { O }}\end{array}$ \\
\hline 0.5 M EDTA pH 8.0 & $\begin{array}{l}372.3 \mathrm{~g} \text { EDTA are added to } 800 \mathrm{ml} \mathrm{ddH}_{2} 0 . \mathrm{NaOH} \text { pellets are added to } \\
\text { increase the pH and thus allow EDTA to dissolve. The volume is } \\
\text { adjusted to } 1 \mathrm{~L} \text { with } \mathrm{ddH}_{2} \mathrm{O}\end{array}$ \\
\hline SDS Sample buffer & $\begin{array}{l}\text { 3\% SDS, } 125 \mathrm{mM} \text { Tris/HCl pH 6.8, } 50 \mathrm{mM} \text { DTT, } 1 \mathrm{M} \text { sucrose, } \\
\text { Bromphenol blue }\end{array}$ \\
\hline
\end{tabular}




\begin{tabular}{|c|c|}
\hline $\begin{array}{l}\text { 10x SDS-PAGE } \\
\text { running buffer }\end{array}$ & $300 \mathrm{~g}$ Glycine, $60 \mathrm{~g}$ Tris Base, $24 \mathrm{~g}$ SDS, add to $2 \mathrm{~L}$ with ddH20 \\
\hline $\begin{array}{l}\text { Coomassie stock } \\
\text { solution }\end{array}$ & $2 \%(\mathrm{w} / \mathrm{v})$ Coomassie brilliant blue G 250 in $50 \%$ Ethanol \\
\hline 10x Blotting buffer & $\begin{array}{l}100 \mathrm{ml} 10 x \text { SDS-PAGE running buffer, } 200 \mathrm{ml} \mathrm{MeOH}, 0.03 \% \text { SDS, } \\
\text { add to } 1 \mathrm{~L} \text { with } \mathrm{ddH}_{2} \mathrm{O}\end{array}$ \\
\hline 10x TBS buffer & $50 \mathrm{~g}$ Tris/ $\mathrm{HCl}, 14.5 \mathrm{~g}$ Tris Base, $175.2 \mathrm{~g} \mathrm{NaCl}$, add $\mathrm{ddH}_{2} 0$ to $1 \mathrm{~L}$ \\
\hline 10x PBS (pH 7.4) & $137 \mathrm{mM} \mathrm{NaCl}, 2.7 \mathrm{mM} \mathrm{KCl}, 8.1 \mathrm{mM} \mathrm{Na}_{2} \mathrm{HPO}_{4}, 1.76 \mathrm{mM} \mathrm{KH}_{2} \mathrm{PO}_{4}$ \\
\hline Fixation buffer & $50 \% \mathrm{MeOH}, 40 \% \mathrm{ddH}_{2} 0,10 \%$ Acetic Acid \\
\hline 20x E-mix & $\begin{array}{l}20 \mathrm{mM} \text { HEPES pH 7.5, } 250 \mathrm{mM} \text { Sucrose, } 200 \mathrm{mM} \text { Creatine } \\
\text { phosphate, } 10 \mathrm{mM} \text { ATP, } 10 \mathrm{mM} \text { GTP, } 1 \mathrm{mg} / \mathrm{ml} \text { Creatine kinase }\end{array}$ \\
\hline $\begin{array}{l}\text { Gibson Assembly } \\
\operatorname{mix}(2 \mathrm{x})\end{array}$ & $\begin{array}{l}\text { In house-prepared mixture containing 5' Exonuclease, DNA } \\
\text { polymerase and DNA ligase }\end{array}$ \\
\hline Biotinylation buffer & $\begin{array}{l}250 \mathrm{mM} \text { Tris/ } \mathrm{HCl} \mathrm{pH} 7.5,500 \mathrm{mM} \mathrm{NaCl}, 50 \mathrm{mM} \\
\text { ATP, } 62.5 \mathrm{mM} \mathrm{MgCl}{ }_{2}, 1.25 \mathrm{mM} \text { biotin }\end{array}$ \\
\hline $\begin{array}{l}\text { Protein resuspension } \\
\text { buffer }\end{array}$ & $\begin{array}{l}50 \mathrm{mM} \text { Tris/ } \mathrm{HCl} \mathrm{pH} \text { 7.5, 150-500 mM NaCl, 2- } \\
20 \mathrm{mM} \text { imidazole, 0-5 mM DTT }\end{array}$ \\
\hline $\mathrm{Ni}^{2+}$-elution buffer & $\begin{array}{l}50 \mathrm{mM} \text { Tris/ } \mathrm{HCl} \mathrm{pH} 7.5,150-500 \mathrm{mM} \mathrm{NaCl}, \\
500 \mathrm{mM} \text { imidazole, } 250 \mathrm{mM} \text { sucrose, } 0-5 \mathrm{mM} \text { DTT }\end{array}$ \\
\hline $\begin{array}{l}\text { Phage precipitation } \\
\text { buffer }\end{array}$ & $20 \%$ PEG $8000,2.5 \mathrm{M} \mathrm{NaCl}$ \\
\hline Blocking buffer & $50 \mathrm{mM}$ Tris $/ \mathrm{HCl} \mathrm{pH} 7.5,300 \mathrm{mM} \mathrm{NaCl}, 0.1 \%(\mathrm{w} / \mathrm{v}) \mathrm{BSA}$ \\
\hline $\begin{array}{l}\text { Sucrose cushion } \\
\text { buffer }\end{array}$ & $\begin{array}{l}1 \mathrm{M} \text { Ammonium Sulfate, } 1.6 \mathrm{M} \text { sucrose, } 25 \mathrm{mM} \text { Tris- } \mathrm{HCl} \mathrm{pH} \text { 7.5, } 50 \\
\mathrm{mM} \mathrm{MgCl} \\
2,5 \mathrm{mM} \text { DTT }\end{array}$ \\
\hline TNKM buffer & $25 \mathrm{mM}$ Tris- $\mathrm{HCl} \mathrm{pH}$ 7.5, $50 \mathrm{mM} \mathrm{NH}_{4} \mathrm{Cl}, 50 \mathrm{mM} \mathrm{KCl}, 25 \mathrm{mM} \mathrm{MgCl}_{2}$ \\
\hline
\end{tabular}

\subsubsection{Bacterial strains}

Several E. coli strains were used in this study. NEB-10 beta and 5-Alpha (C3019, New England Biolabs) were used for cloning purposes. Strains BLR (Novagen) and NEB Express (New England Biolabs) were main recombinant protein expression strains. Phage display competent cells SS320 and TG1 (Lucigen) are electrocompetent cell strains and they were used to produce phages for phage display. Thermus thermophilus bacterial strain was obtained from (RAS, Russia). All the electro-competent cells were harvested and prepared by Gabriele Hawlitschek and Uwe Hoffman according to Sambrook, J., Russell 2001 . 


\subsubsection{Bacterial media}

Table 5.3 Bacterial media

\begin{tabular}{|c|c|}
\hline Media & Composition \\
\hline TB & $\begin{array}{l}12 \mathrm{~g} \text { tryptone, } 24 \mathrm{~g} \text { yeast extract, } 0.4 \%(\mathrm{v} / \mathrm{v}) \text { glycerol, } 72 \mathrm{mM} \mathrm{K}_{2} \mathrm{HPO}_{4} \text {, } \\
17 \mathrm{mM} \mathrm{KH}_{2} \mathrm{PO}_{4}\end{array}$ \\
\hline $2 \mathrm{YT}$ & $16 \mathrm{~g}$ tryptone, $10 \mathrm{~g}$ yeast extract, and $5 \mathrm{~g} \mathrm{NaCl}$ per $1 \mathrm{~L} \mathrm{H}_{2} \mathrm{O}$ \\
\hline LB & $10 \mathrm{~g}$ tryptone, $5 \mathrm{~g}$ yeast extract, and $10 \mathrm{~g} \mathrm{NaCl}$ per $1 \mathrm{~L} \mathrm{H}_{2} \mathrm{O}$ \\
\hline SOB & $\begin{array}{l}20 \mathrm{~g} \text { tryptone, } 5 \mathrm{~g} \text { yeast extract, } 10 \mathrm{mM} \mathrm{NaCl}, 2.5 \mathrm{mM} \mathrm{KCl}, 10 \mathrm{mM} \\
\mathrm{MgCl}, 10 \mathrm{mM} \mathrm{MgSO}_{4} \text { per } 1 \mathrm{~L} \mathrm{H}_{2} 0\end{array}$ \\
\hline Tth medium & $40 \mathrm{~g}$ tryptone, $20 \mathrm{~g}$ yeast extract, $10 \mathrm{~g} \mathrm{NaCl}, 8 \mathrm{ml}$ of $1 \mathrm{M} \mathrm{NaOH}$ per $5 \mathrm{~L}$ \\
\hline
\end{tabular}

All media were prepared and autoclaved in the lab. Antibiotics Ampicillin, Kanamycin, Trimetoprim, and Spectinomycin were used at concentrations 10-100 $\mu \mathrm{g} / \mathrm{ml}$.

\subsubsection{Software}

Table 5.4 Software

\begin{tabular}{|l|l|}
\hline Software & Developer \\
\hline Lasergene Suite 9.0 & DNASTAR, USA \\
\hline Oligo 7.58 & Molecular Biology Insights, USA \\
\hline Odyssey & LI-COR Biosciences, USA \\
\hline Scaffold 4 & Proteome Software, USA \\
\hline LASAF & Leica, Germany \\
\hline Python 2.7.10 & Python Software Foundation \\
\hline Mac OS X Yosemite Version 10.10.5 & Apple, USA \\
\hline CFX ManagerTM & Bio-Rad Laboratories Inc., USA \\
\hline MacPyMol & Schrödinger LLC, USA \\
\hline
\end{tabular}




\begin{tabular}{|l|l|}
\hline Adobe Illustrator + Photoshop CS5.1 & Adobe Systems Inc., USA \\
\hline Microsoft Excel for Mac 2011 & Microsoft Corp., USA \\
\hline
\end{tabular}

\subsection{Standard methods in molecular biology}

All standard molecular biology methods were performed as described by Sambrook, J., Russell 2001. All phage display methods and techniques were performed as described by Clarkson \& Lowman 2004.

\subsubsection{E. coli transformation}

Electroporation was a standard method of transformation E. coli cells. For that, $40 \mu 1$ of electrocompetent E. coli cells were mixed with $50 \mathrm{ng}$ of plasmid DNA in an electroporation cuvette (Biorad, Hercules, CA, USA). Electroporation was done using a Gene Pulser (BioRad, Burlington, USA). The cells were then placed on ice and recovered with $1 \mathrm{ml}$ of $2 \mathrm{YT}$ medium supplemented with $0.5 \%(\mathrm{w} / \mathrm{v})$ glucose. After that cells were carefully resuspended, transferred to a fresh eppendorf tube and placed into an incubator for $1 \mathrm{~h}$ at $37^{\circ} \mathrm{C}$ while shaking. Next, desired volume of the cells suspension (usually $50 \mu \mathrm{l}$ ) was plated onto agar plates with appropriate antibiotics and left to grow over night at $37^{\circ} \mathrm{C}$. Transformed E. coli colonies were individually picked for plasmid DNA preparations and/or protein expression.

\subsubsection{DNA purification from $E$. coli}

Plasmid DNA was extracted either for analytical purposes (NucleoSpin Plasmid Kit, Marcherey Nagel, Germany) or on preparative scale (NucleoBond PC100 Kit, Marcherey Nagel, Germany) according to manufacturer's directions. Upon DNA extraction NanoDrop 2000C spectrophotometer (PeqLab, Germany) was used to determine DNA concentrations.

\subsubsection{DNA restriction digestion and ligation}

DNA restriction digestion enzymes and appropriate buffers were obtained from New England Biolabs, USA. DNA was digested for $1-2 \mathrm{~h}$ at $37^{\circ} \mathrm{C}$ according to manufacturer's instructions. To reduce re-annealing of the vector and consequently background levels of cloning, vectors were dephosphorylated using Fast Alkaline Phosphatase (Fermentas) for 30 minutes at $37^{\circ} \mathrm{C}$. For a standard ligation 30 fmoles of vector were mixed with 90 fmoles 
of insert fragments and supplemented with T4 DNA ligase (produced in-house by Steffen Frey, Department of Cellular Logistics) and 1x Ligation Buffer and diluted to $10 \mu 1$ with $\mathrm{H}_{2} \mathrm{O}$. The reaction was incubated at $37^{\circ} \mathrm{C}$ for $30 \mathrm{~min} .1 \mu 1$ of the ligation reaction was used to transform electro competent $E$. coli cells. A negative control reaction without any insert served as a negative control.

\subsubsection{Agarose gel electrophoresis and DNA isolation}

Agarose gel electrophoresis was used to isolate desired DNA fragments after restriction digestion. In short, DNA fragments were separated on agarose gels in 1xTAE running buffer. Agarose concentrations, usually between 1-1,5\%, varied depending on DNA size that had to be separated. To visualize DNA $0.05 \mu \mathrm{g} / \mathrm{ml}$ ethidium bromide was added to warm agarose prior solidifying. All DNA samples were thoroughly mixed with Orange G loading buffer before loading onto an agarose gel. To extract DNA out of agarose blocs desired DNA bands were cut out and isolated with Zymoclean Gel DNA recovery kit (Zymo Research, USA) according to manufacture's protocols. DNA concentration was measured on the NanoDrop 2000C spectrophotometer (PeqLab, Germany).

\subsubsection{Polymerase chain reaction}

PCR is a standard method to amplify or mutagenize DNA fragments from a template. Inhouse prepared "Pfus Triple Mix" for PCR was used. This mix contains Pfus polymerase, phosphatase and dUTPase. PfuS is a fusion of thermostable DNA-binding domain (Sac7d or Sso7d) with Pfu polymerase. PfuS has a speed of about $3.3 \mathrm{~kb} / \mathrm{min}$ and high fidelity proofreading. The composition of a standard PCR reaction is shown in table 1.5.

Table 5.5 PCR reaction composition

\begin{tabular}{|l|l|}
\hline Ingredient & Amount \\
\hline PfuS triple mix & $1 \mu 1$ \\
\hline DMSO & $2 \mu 1$ \\
\hline dNTP mix & $10 \mu 1$ \\
\hline $10 x$ PfuS buffer & $10 \mu 1$ \\
\hline Reverse primer $(100 \mu \mathrm{M})$ & $1 \mu 1$ \\
\hline
\end{tabular}




\begin{tabular}{|l|l|}
\hline Forward primer $(100 \mu \mathrm{M})$ & $1 \mu 1$ \\
\hline DNA template & $50 \mathrm{ng}$ \\
\hline $\mathrm{ddH}_{2} \mathrm{O}$ & up to $100 \mu \mathrm{l}$ \\
\hline
\end{tabular}

Annealing temperature of $62^{\circ} \mathrm{C}$ would usually result in adequate PCR product production. In special cases a gradient PCR was used to determine the optimal annealing temperature.

Table 5.6 PCR reaction conditions

\begin{tabular}{|c|c|c|c|}
\hline Step & Temperature, ${ }^{\circ} \mathrm{C}$ & Duration, min & Number of cycles \\
\hline Pre-warming & 98 & 1 & - \\
\hline Denaturation & 98 & 0.5 & \multirow{3}{*}{25} \\
\hline Annealing & 62 & 0.5 & \\
\hline Elongation & 68 & $1 \mathrm{~min}$ per $3 \mathrm{~kb}$ & \\
\hline Final elongation & 68 & 5 & - \\
\hline
\end{tabular}

\subsubsection{Error-prone PCR reaction}

Error-prone PCR was introduced by Vartanian et al. 1996 and it relies on incorporation of mutagenic dNTP analogs (8-oxo-dGTP, dPTP) into a PCR product. Error prone PCR kits were purchased from Jena Bioscience (Germany) and the reaction was carried out according to manufacturers recommendations. The dNTP analogs were added directly to the PCR reaction mixture. The mutagenic dNTPs must have been eliminated in a follow up PCR reaction where only natural dNTPs were present.

\subsubsection{Gibson assembly}

Gibson assembly (Gibson, 2011) is a technique that allows a single step seamless assembly of multiple overlapping DNA fragments. It relies on function of three enzymes: 5' exonuclease, a DNA polymerase, and a DNA ligase. 5' exonuclease treatment yields single-stand DNA overhangs that anneal specifically. These overhangs are basically 
identical DNA stretches of about $\sim 15$ nucleotides long on both a vector and an insert. After that overhangs anneal any single-strand regions are filled by DNA polymerase. Finally the nicks are covalently joined by the DNA ligase thus producing a circularized DNA molecule. The enzyme mix used for the Gibson assembly was produced in the lab. In short, 30 fmoles of a vector backbone was mixed with 60 fmoles of an insert in a PCR tube and supplemented with $2.5 \mu \mathrm{l}$ of the Gibson assembly mix. The reaction was incubated for about $30 \mathrm{~min}$ at $50^{\circ} \mathrm{C}$. For background control, vector only was incubated with the Gibson assembly mix in parallel. At the end $40 \mu 1$ of electrocompetent cells were transformed with $0.8 \mu 1$ of the Gibson assembly reaction and plated onto agar plates with respective antibiotics.

\subsubsection{Oligonucleotide design and DNA sequencing}

All nucleotides used in this work were designed using SeqBuilder sowtware (DNAStar, USA) and Oligo 7.58 (Molecular Biology Insights, USA). Oligonucleotides were synthesized by Sigma-Aldrich (Germany) and dissolved to the final concentration of 100 $\mu \mathrm{M}$ in distilled $\mathrm{H}_{2} \mathrm{O}$. All plasmids were sequenced at Seqlab (Germany) with self-designed primers. The plasmid sequencing analysis was done with the help of SeqMan software (DNAStar, USA).

\subsection{Protein expression and purification}

\subsubsection{Recombinant protein expression in E. coli}

All recombinant proteins used in this study were expressed and purified in E. coli strains described in the section "Bacterial strains". The strain and expression conditions were determined for each protein experimentally to optimize the yield. A standard protocol for protein was carried as follows, E. coli cells were transformed with a desired vector and plated onto agar plates, then individual colonies were picked for further use. Single colonies were pre-grown in $100 \mathrm{ml}$ 2YT medium supplemented with antibiotics overnight at $30^{\circ} \mathrm{C}, 90 \mathrm{rpm}$. After that the culture was diluted six-fold with pre-warmed medium. When $~ 1$ OD had been reached the culture was kept for at least $1 \mathrm{~h}$ at the desired expression temperature, usually between $18^{\circ} \mathrm{C}$ and $25^{\circ} \mathrm{C}$. Next, the culture was induced with IPTG $(0.1-0.2 \mathrm{mM})$ and induced bacteria were incubated for $4-8 \mathrm{~h}$ while shaking at $90 \mathrm{rpm}$. Prior cell harvesting PMSF and EDTA were added as protease inhibitors. Finally, bacterial cells were pelleted for $15 \mathrm{~min}$ at $7500 \mathrm{rpm}$ at $4^{\circ} \mathrm{C}$ and the pellet was re-suspended 
in a native buffer $(50 \mathrm{mM}$ Tris $7.6 \mathrm{pH}, 130-300 \mathrm{NaCl}, 2-10 \mathrm{mM}$ imidazole, and 0-3 $\mathrm{mM}$ DTT). Re-suspended cells were frozen in liquid nitrogen until further use. Probes for protein SDS PAGE Gel analysis were taken before and after induction.

\subsubsection{Recombinant protein purification}

In order to lyse E. coli cells they were frozen and unfrozen in the re-suspension buffer and sonicated (Sonifier 450, Branson, UK) on ice for about $5 \mathrm{~min}$ and $45 \%$ duty cycle. The lysed cells were ultra-centrifuged (WX Ultracentrifuge, T647.5 rotor, Sorvall) for $1.5 \mathrm{~h}$ at $36000 \mathrm{rpm}$ at $4^{\circ} \mathrm{C}$. The cell lysate was either directly used for protein purification by affinity chromatography (Ni-chromatography) or supplemented with $250 \mathrm{mM}$ sucrose and stored at $-80^{\circ} \mathrm{C}$.

\subsection{3 $\mathrm{Ni}^{2+}$ chromatography protein purification}

All constructs for protein expression used in this study were fused to a His-tag. The length of the His-tag was either 6,10 , or 14 Histidines. $\mathrm{Ni}^{2+}$-EDTA matrixes that were used for protein purification were produced in the lab by Prof. Dirk Görlich. Usually, 20\% or 24\% substituted $500 \AA \mathrm{Ni}^{2+}$-EDTA silica matrix was preferred for purification of small proteins. Before starting large scale protein purification a test binding experiment was performed on a small scale using Mobi-Cols (MoBiTech, Germany). These experiments allowed determination of binding capacity of the matrix to a given protein.

To purify His-tagged proteins $1-2 \mathrm{ml}$ of $\mathrm{Ni}^{2+}$-EDTA matrix was loaded onto a chromatography column and equilibrated with the re-suspension buffer. Usually 3 column volumes are sufficient to equilibrate the column. Clear lysate of E. coli cells obtained as described in the "Recombinant protein purification" section was mixed with the preequilibrated Ni-matrix. The binding of His-tagged protein to the Ni-matrix was at $4^{\circ} \mathrm{C}$ for $2 \mathrm{~h}$ while rotating. The liquid chromatography column was then loaded with the lysate$\mathrm{Ni}^{2+}$-matrix suspension, the flow through was collected for further analysis. Subsequently

the column was washed three times with the washing buffer which usually has the same composition as the resuspension buffer except for higher (20-25 mM) imidazole concentration. After the washing protein was eluted from the column in a step wise manner. The elution buffer contains $0.5 \mathrm{M}$ imidazole and allows optimal elution of Histagged protein. Individual $1 \mathrm{ml}$ fractions were analyzed for protein content using Amido Black Quick Staining and protein containing fractions were pulled together. Samples of $E$. 
coli supernatant, insoluble fraction, $\mathrm{Ni}^{2+}$-chromatography-flow-through, and the eluate were taken for further analysis on a SDS PAGE gel.

\subsection{4 $\mathrm{Ni}^{2+}$ chromatography with on column protein cleavage}

Protein purification in the manner described in the "Ni2+ chromatography protein purification" section results in a protein production with $\mathrm{N}$-terminal tags. On column protein cleavage was performed if purification of a protein without a tag was desired. After several washing steps a protease (SUMOstar or brSUMO prepared by Dr. Steffen Frey) was added in concentrations between $100 \mathrm{nM}$ and $500 \mathrm{nM}$. The mixture was incubated for $1 \mathrm{~h}$ at $4^{\circ} \mathrm{C}$. The cleaved protein was collected by addition of re-suspension buffer in a stepwise manner. Collected fractions $(1 \mathrm{ml})$ were analyzed with Amido Black Quick Staining for protein content and pooled together. The protein concentration was determined by NanoDrop and Protean (Lasergene suit, USA). The purity of proteins was determined on SDS PAGE gels. For storage, eluted proteins were supplemented with $250 \mathrm{mM}$ sucrose and frozen in liquid nitrogen.

\subsubsection{In vivo biotinylation of recombinant proteins}

Biotinylation of recombinant proteins was achieved by co-expression of BirA enzyme together with a protein of interest in E. coli cells. To ensure selectivity both plasmids carried different selective markers (antibiotic resistances). The growth medium had to be supplemented with pure biotin (Sigma, Germany). $\mathrm{Ni}^{2+}$ purified proteins were tested for successful biotinylation by a test binding to the Dyna beads MyOne Streptavidin T1 (Life Technologies, USA).

\subsubsection{Size exclusion chromatography}

To re-buffer purified proteins, mainly to get rid of imidazole that is sometimes undesirable for biological assays, size exclusion chromatography was the method of choice. Nap5 and PD-10 (GE Healthcare, USA) were used for the purpose of re-buffering. A column was first equilibrated with a buffer of choice and the sample was loaded onto it. The sample protein was eluted in a stepwise manner and protein containing fractions were pulled together. Sample protein content was checked on a SDS-PAGE with a subsequent Coomassie staining. 


\subsubsection{Purification of 305 and 505 Thermus thermophilus ribosomal subunits}

Ribosomes were purified from Thermus thermophilus as described by Korostelev et al. 2006. In short, to purify $70 \mathrm{~S}$ ribosomes Thermus thermophilus cells were grown to $0,5-0,7$ $\mathrm{OD}$ at $72^{\circ} \mathrm{C}$ while shaking. Cells were centrifuged at $8000 \mathrm{rpm}$ at $4^{\circ} \mathrm{C}$ for $15 \mathrm{~min}$. Then they were washed twice with the 2x TNKM buffer supplemented with $7 \mathrm{mM}$ DTT and then re-suspended in 2x TNKM with RNAse free DNAseI (Jena Bioscience, Germany). At this point re-suspended cells were frozen if needed. A French press (Avestin, Canada) was used to disrupt cellular membranes. Cells were usually treated for two cycles at settings recommended by the manufacturer. Cell debris were removed by centrifugation $(30000$ $\mathrm{rpm}, 4^{\circ} \mathrm{C}, 1 \mathrm{~h}$ ) (Sorval Wx Ultra ultracentrifuge). To further purify $70 \mathrm{~S}$ ribosomes supernatant was carefully loaded onto a sucrose cushion and ultracentrifuged for $16 \mathrm{~h}$ (36000 rpm, $4^{\circ} \mathrm{C}$ ) (Sorval Wx Ultra ultracentrifuge). After that the supernatant was discarded and a transparent ribosomal pellet was washed twice with 1x TNKM buffer supplemented with $1 \mathrm{M}$ ammonium acetate and dissolved in $10 \mathrm{ml}$ of the same buffer. Next, crude 70S ribosomes were loaded onto a HiTrap Butyl column (GE Gealthcare Life Science, Germany), which was equilibrated with 1x TNKM buffer supplemented with $1 \mathrm{M}$ ammonium sulfate. After loading the ribosomes onto the column it was intensively washed with 1x TNKM buffer supplemented with $0.8 \mathrm{M}$ ammonium sulfate. In order to elute ribosomes from the column a reversed gradient of ammonium sulfate was applied from 0.8 $\mathrm{M}$ to 0 and $1.5 \mathrm{ml}$ fractions were collected. Protein containing fractions were analyzed by gel electrophoresis and pulled together.

\subsubsection{SDS polyacrlylamide gel electrophoresis}

Sodium dodecyl sulfate polyacrylamide gel electrophoresis (SDS-PAGE) (Laemmli, 1970) is a standard method to separate proteins according to their molecular weight. All the gels used in this study were 8-12\% and prepared by Gabriele Hawlitschek. Protein samples to be loaded onto a gel were supplemented with 5x SDS sample buffer and boiled at $95^{\circ} \mathrm{C}$ for $5 \mathrm{~min}$. Protein samples were run at $400 \mathrm{~V}$ and $50 \mathrm{~mA}$ for 1 hour 10 minutes. Gels were stained with Coomassie staining solution and consequently washed with distilled water to remove any background staining. 


\subsubsection{Maleimide-flurophore conjugation to proteins with free -SH groups}

Reaction between thiols and maleimides is widely used for bio-conjugation and labeling of biomolecules such as proteins and peptides. Prior labeling proteins had to be reduced with $10 \mathrm{mM}$ DTT for $20 \mathrm{~min}$. The samples were then run on a gel filtration column (Sephadex G25) which was equilibrated with the following de-gassed buffer: 13,3 $\mathrm{mM} \mathrm{KH}_{2} \mathrm{PO}_{4}, 6,7$ $\mathrm{mM} \mathrm{K} \mathrm{HPO}_{4}, 300 \mathrm{mM} \mathrm{NaCl}, 1 \mathrm{mM}$ EDTA, $250 \mathrm{mM}$ sucrose. The protein sample was eluted using the same buffer and protein containing fractions were collected and pulled together.

A maleimide-fluorophore (normally dissolved at $10-20 \mathrm{mM}$ in DMF) was added to an empty Eppi and placed on ice. The reduced protein sample was added in 1:1,2 molar concentration. The $\mathrm{pH}$ of the reaction was raised by addition of $100 \mathrm{mM} \mathrm{K} \mathrm{HPO}_{4}$ or 100 $\mathrm{mM}$ Tris/ $\mathrm{HCl} \mathrm{pH} 7,3$ to start the reaction. The mixture was incubated on ice for $60-120$ minutes. In order to separate free fluorophores, Sephadex G25 gel-filtration columns were used or Ni-chelate chromatography, if the target protein still contains a His-tag. Finally, the extend of labeling was calculated and a quality check was performed on a SDS gel.

\subsubsection{Biotinylation of $70 \mathrm{~S}$ Thermus thermophilus ribosomes}

Purified ribosomed prepared as described in the "Purification of $30 \mathrm{~S}$ and 50S Thermus thermophilus ribosomal subunits" were reduced using $10 \mathrm{mM}$ DTT for 30 minutes on ice. Reduced ribosomes were re-buffered on PD10 columns (GE Gealthcare Life Science, USA) into a DTT free buffer. DTT free ribosomes were mixed with Maleimide-PEG-H (Sigma-Aldrich, Germany). As a positive control, to estimate accessibility of free - $\mathrm{SH}$ groups on $70 \mathrm{~S}$ Tth ribosomes and degree of binding of Maleimide-PEG-H, a separate sample was incubated with Maleimide-Alexa 488. Samples were incubated on ice for $1 \mathrm{~h}$. In order to get rid of free Maleimide-PEG-H or Maleimide-Alexa 488 the samples were rebuffered on PD10 columns (GE Gealthcare Life Science, USA). To estimate degree of labeling small amount of biotinylated 70S ribosomes was immobilized onto Dyna beads MyOne Streptavidin T1 (Life Technologies, USA). The non-bound, flow through, elution and beads fractions were analyzed on an SDS-PAGE gel. 


\subsubsection{Protein-rRNA cross-linking}

In collaboration with the group of Prof. Bohnsack an rRNA-nanobody cross-linking was performed as previously described (Bohnsack et al., 2012). In brief, nanobody-rRNA UV cross-linked complex was partially digested with RNAse (1U RNAceIT, Agilent) and complexes were purified under denaturing conditions $(6 \mathrm{M}$ guanidinium- $\mathrm{HCl})$ on $\mathrm{Ni}^{2+}-\mathrm{NTA}$ column (Qiagen). Co-purified rRNA was radioactively labeled by T4 PNK (Thermo Scientific) and ${ }^{32} \mathrm{P}-\gamma$-ATP (Perkin Elmer). Then RNA-protein complexes were separated on NuPAGE gel electrophoresis and transferred onto a nitrocellulose membrane. The membrane was then exposed to an X-ray film and radioactive signals were detected. In order to identify specific interaction sites of nanobody with rRNA 3' and 5' adapters were ligated with rRNA. Next a cDNA library was prepared by reverse transcription of rRNA using Superscript III Reverse Transcriptrase (Thermo Fisher, US). The amplification was done using randomized five-nucleotide primers to ensure PCR templates are distinguished. Lastly sequenced results were mapped onto the $23 \mathrm{~S}$ rRNA.

\subsection{Library construction and phage display}

\subsubsection{Animal immunization}

Alpacas (Vicugna pacos) were immunized by injecting $0.5-1 \mathrm{mg}$ of protein over a period of 4 weeks for 3-4 times. All antigens were either recombinantly expressed in E. coli or purified from a native source. The antigens were solubilized in a physiological buffer that consists of $20 \mathrm{mM}$ Tris/ $\mathrm{HCl} \mathrm{pH}$ 7.5, $150 \mathrm{mM} \mathrm{NaCl}, 250 \mathrm{mM}$ Sorbitol. Two adjuvants were used for the immunization: a mild AS03-like adjuvant (oil-in-water emulsion) and a peptide adjuvant (N-Acetylmuramyl-L-alanyl-D-isoglutamine hydrate; Sigma-Aldrich, USA). Antigen injection sites were shaved and locally anesthetized prior injection. The injections and blood collection was performed by Dr. Ulrike Teichmann.

\subsubsection{Lymphocyte isolation and total RNA preparation}

Four days after the final antigen injection $100 \mathrm{ml}$ of blood were collected from the jugular vein from an immunized animal. A special vacuum bottle was pre-filled with $100 \mathrm{ml}$ of blood dilution buffer (1x PBS, 0.4\% glucose, $25 \mathrm{mM}$ EDTA) which prevents blood coagulation. Density gradient centrifugation (Leucosep, Greiner Bio-One) was the method of choice to isolate blood peripheral lymphocytes. After the centrifugation blood 
peripheral lymphocytes form a distinct white cell layer. These cells were washed with blood dilution buffer and collected for further total RNA extraction. The total RNA was extracted as described by Chomczynski and Sacchi (Chomczynski and Sacchi, 1987). The aforementioned method is based on the fact that under acidic conditions total RNA stays in the aquatic phase, whereas the total DNA and proteins remain the lower organic phase. RNA concentration was measured using NanoDrop 2000C spectrophotometer (PeqLab, Germany). RNA quality was verified by agarose gel electrophoresis and capillary gel electrophoresis using Bioanalyzer RNA 6000 Nano kit (Agilent Technologies, USA). Total RNA was stored in $75 \%$ ethanol at $-80^{\circ} \mathrm{C}$ until further use. Lymphocyte isolation and RNA preparation was carried out by Jens Krull.

\subsubsection{Nanobody library construction}

To construct a nanobody cDNA library, $30 \mu \mathrm{g}$ of total RNA was used in SuperScript III First-Strand Synthesis System (Life Technologies, USA) with $\mathrm{CH} 2$ domain specific primers for IgG (Dumoulin et al., 2002). To specifically amplify VHH domain encoding genes nested PCR was used. $5 \mu 1$ of cDNA from a previous reaction were used to set 24 PCR reactions with primers (AlpVh-L, AlpVHHR1, AlpVHHR2) that anneal in the leader sequence and VHH-specific hinge regions (Maass et al., 2007). The $\sim 400 \mathrm{bp}$ PCR products were analyzed and purified on an agarose gel. Given PCR products served as a template for a consequent PCR reaction in order to introduce restriction sites or Gibson assembly overhangs. For the construction of the nanobody library against Thermus thermophilus 70S ribosomes two restriction sites (Bam/NotI) were introduced. The PCR product was purified with MSB spin PCRapace kit (Stratec, Germany) and digested with respective enzymes over night at $16^{\circ} \mathrm{C}$. The vector for cloning the library was prepared the same way in parallel.

Alternatively Gibson assembly was used to clone the nanobody library into an appropriate vector. As in the case of restriction enzyme cloning complementary overhangs of $\sim 15$ nucleotides have to be introduced upstream and downstream of conserved nanobody regions. The very same overhangs have to be introduced also into the cloning vector by PCR. For the construction of nanobody library against Lamin B3 three forward primers PT718-720 and three reverse primers PT721-723 were combined and 9 PCR reactions were carried out. The products of all PCR reactions were purified with the help of MSB spin PCRapace kit (Stratec, Germany) and mixed in equimolar concentrations. 


\subsubsection{Transformation of nanobody library}

The phagemid pMG40 was used as a vector for nanobody library cloning. $25 \mu \mathrm{g}$ of pMG40 phagemid vector was digested with Bam/NotI restriction enzymes in presence of alkaline phosphatase (FastAP, Thermo Scientific, US) overnight at $37^{\circ} \mathrm{C}$. In a similar way $15 \mu \mathrm{g}$ of nanobody library PCR product was digested with the same enzyme pair overnight at $37^{\circ} \mathrm{C}$. $6 \mu \mathrm{g}$ of digested vector was combined with $3 \mu \mathrm{g}$ of nanobody library and ligated at $16^{\circ} \mathrm{C}$ overnight in the smallest possible volume. The ligation reaction was purified with MSB spin PCRapace kit.

In case of Gibson assembly similar amounts of the vector and the insert were taken for the reaction and supplemented with a $2 x$ Gibson reaction mix (prepared in the lab) and incubated for $30 \mathrm{~min}$ at $50^{\circ} \mathrm{C}$. In order to get rid of the salt that might compromise the efficiency of transformation the Gibson assembly reaction was purified with MSB spin PCRapace kit.

Ligated and purified plasmids were directly used for transformation. For this purpose SS320 E. coli cells were routinely used as they are electrocompetent and deliver the best results. On average 3-4 transformation reactions were made each with $\sim 350 \mu 1$ of SS320 cells and $\sim 500 \mathrm{ng}$ of assembled vector. The purified DNA was added to the cells pipetted up and down avoiding bubbles and chilled on ice. The mixture is then transferred to an electroporation cuvette and electroporated on GenePulser (Biorad, US) following the manufacturer instructions. The cells were immediately rescued by addition of $1 \mathrm{ml}$ of SOC media and transferred to a $250 \mathrm{ml}$ baffled flask.

In order to determine the library complexity a series dilution of the electroporated cells was plated onto the plates with the appropriate antibiotic. The transformation efficiency usually ranged in $2-8 \times 10^{8} \mathrm{cfu}$ per $\mu \mathrm{g}$ of DNA. This allowed construction of highly complex libraries with $2-4 \times 10^{9}$ transformations.

The electroporation cuvettes were thoroughly rinsed once more to collect all the cells. 25 $\mathrm{ml}$ of the SOC media was transferred to the cells and incubated for about $1 \mathrm{~h}$ at $37^{\circ} \mathrm{C}$ while shaking. After $1 \mathrm{~h}$ incubation time the culture was kept without shaking for $\sim 15 \mathrm{~min}$ to allow the growth of F pili on bacterial cells. Subsequently the helper phage M13K07 was added, such that the final concentration of the helper phage in the culture was $\sim 10^{10}$ per ml. Cells were kept still for another 30 min to ensure high infectivity of the helper phage. At the end the culture was transferred to a large $5 \mathrm{~L}$ flask and filled up to $500 \mathrm{ml}$ with $2 \mathrm{YT}$ 
media supplemented with appropriate antibiotics. The culture was then incubated overnight at $37^{\circ} \mathrm{C}$, while shaking.

\subsubsection{Harvest and purification of bacteriophages}

A cell culture prepared as described in the "Transformation of nanobody library" section was centrifuged for $10 \mathrm{~min}$ at $7000 \mathrm{rpm}$ to pellet E. coli cells. The supernatant was decanted to a new centrifugation tube. Then $60 \mathrm{ml}$ of $20 \%$ PEG-8000 in $2.5 \mathrm{M} \mathrm{NaCl}$ was added which is used to precipitate phages. The supernatant was incubated for about $30 \mathrm{~min}$ at the room temperature. At the end of the incubation time phages were centrifuged for 15 min at $7500 \mathrm{rpm}$. After pelleting the phages form a white pallet on the bottom of the tube, which still contains bacterial contaminants. Tubes were inverted and the excess of liquid was drained on paper towels. The white phage pellet was re-suspended in $25 \mathrm{ml}$ of $50 \mathrm{mM}$ Tris/ $\mathrm{HCl} \mathrm{pH} 7.5,300 \mathrm{mM} \mathrm{NaCl}$ using a $25 \mathrm{ml}$ glass pipet. The phage suspension was centrifuged in SS-34 rotor (Sorvall/Thermo Scientific, USA) for $10 \mathrm{~min} 15000 \mathrm{~g}$ at $4^{\circ} \mathrm{C}$ to remove bacterial debris and aggregates. The supernatant containing phages was carefully poured into a $50 \mathrm{ml}$ falcon tube that contains $5 \mathrm{ml}$ of $20 \%$ PEG-8000 in $2.5 \mathrm{M} \mathrm{NaCl}$ and incubated for $30 \mathrm{~min}$. Afterwards the tubes were centrifuged in the F13 rotors (Sorvall/Thermo Scientific, USA) for $15 \mathrm{~min}, 2200 \mathrm{~g}$, at $4^{\circ} \mathrm{C}$. Then the tubes were inverted and the liquid excess was drained with a paper towel. Similarly as in previous steps the white visible pellet was re-suspended in $5 \mathrm{ml}$ of $50 \mathrm{mM}$ Tris/ $\mathrm{HCl} \mathrm{pH} \mathrm{7.5,300} \mathrm{mM} \mathrm{NaCl}$. To ensure the mild re-suspension of phages the tubes were placed on the tumbler for $1 \mathrm{~h}$ at $4^{\circ} \mathrm{C}$ while rolling. Finally, to remove small amounts of bacterial contaminants or aggregates the phages were centrifuged one more time in a table top centrifuge (Eppendorf, Germany) for $10 \mathrm{~min}, 13000 \mathrm{rpm}$ at $4^{\circ} \mathrm{C}$. At the end of the centrifugation the supernatant was transferred to a fresh Eppi and stored until further use.

\subsubsection{Phage particles quantification}

To quantify phage concentrations two main methods were used: spectrophotometry or phage tittering. It is worth mentioning that the clear advantage of the latter is that the phage titering allows quantification of viable infective phages.

First, to quantify phages by titering trimethoprim plates were prepared. In parallel TG1 cells were plated onto plates with no antibiotics. A single colony was picked and grown in $\sim 10-15 \mathrm{ml}$ of 2 YT medium with no antibiotics. The TG1 cells were grown until $\sim 0.6$ 
OD600. For each sample to be titrated $90 \mu 1$ of $2 \mathrm{YT}$ was aliquoted into 6-8 wells of a $96 \mathrm{~F}$ plate. $10 \mu \mathrm{l}$ of phages were added to the first well and mixed well with a pipette. A series dilution was performed by transferring $10 \mu \mathrm{l}$ of phages from each well to the next one, using a multichannel pipette. Importantly, clean pipette tips must be used for each transfer. In a second 96F plate $90 \mu 1$ of fresh log phase were aliqouted. Next $10 \mu 1$ were transferred from the plate containing phage titer into the plate with TG1 cells and mixed well. The plates were incubated for $\sim 1 \mathrm{~h}$ at $37^{\circ} \mathrm{C}$. Trimethoprim containing plates were dried at $37^{\circ} \mathrm{C}$ for $1 \mathrm{~h}$. Then using a multichannel pipette $10 \mu \mathrm{l}$ from each well were plated onto a predried trimethoprim plate and allowed to soak into agar. The plates were incubated overnight at $37^{\circ} \mathrm{C}$. Colonies were counted at an appropriate dilutions and $\mathrm{cfu} / \mathrm{ml}$ was calculated for each library and controls.

Another wide spread method to quantify phages employs spectrophotometry measurements. Spectrophotometry allows fast and convenient quantification of concentrations phage solutions. To determine phage concentration the absorbance of the phage solution was measured at $269 \mathrm{~nm}$ and $320 \mathrm{~nm}$. M13K07 phages have the absorbance peak at $269 \mathrm{~nm}$, while their low absorbance at $320 \mathrm{~nm}$ allows to use this value to correct for contaminating non-phage particles. The formula below is used to quantify phage particles (Day and Wiseman, 1978).

$$
\mathrm{c}[\mathrm{phage} / \mathrm{ml}]=\left(\mathrm{A}_{269 \mathrm{~nm}}-\mathrm{A}_{320 \mathrm{~nm}}\right) \cdot 6 \cdot 10^{16} / \text { phagemid size }(\mathrm{bp})
$$

\subsubsection{Selecting of antigen-specific antibodies}

Antigen specific antibodies from phage antibody libraries were isolated by recursive rounds of bio-panning. In a nutshell, bio-panning is a technique that allows affinity based selection of binders to a given antigen.

The antigens used in this work were either recombinantly expressed or purified from native sources. Recombinantly produced proteins were expressed in E. coli with a His14-AviTag $\mathrm{N}$-terminal tag. All protein tags were separated by short flexible linkers to minimize steric hindrance effects. Additionally, brSUMO or SUMOstar serve the purpose of a cleavable module, which allows convenient and specific elution of antigen-bound phages.

In this study two types of panning were performed. In order to capture most of the phages that display desired nanobodies the very first round of panning was on-column. All the consecutive rounds of bio-selection were in solution. In case of on-column bio-panning 
25 pmoles of a biotinylated antigen was pre-immobilized onto $1 \mathrm{mg}$ of magnetic Dynabeads MyOne Streptavidin T1 (Life Technologies, USA) in $50 \mathrm{mM}$ Tris/HCl pH 7.5, $300 \mathrm{mM} \mathrm{NaCl}$, and $0.1 \%$ BSA. The same amount of beads but without any antigen served as a negative control. All non bound antigen was washed off by three washing steps with the buffer mentioned above. Next, the beads were incubated with $10^{13}$ phages from the initial phage library. Unbound phages were discarded and beads were washed several times in order to remove all non-specifically bound phages. Lastly antigen-bound phages were specifically eluted by $100 \mathrm{nM}$ brSUMO protease addition.

Bio-panning in solution was done in a similar manner with the only exception being that the antigen and the phage library were premixed and incubated for $1 \mathrm{~h}$. Then they were added to $1 \mathrm{mg}$ of magnetic Dynabeads and all the consecutive steps were performed as in panning on-column. Ten microliters of the eluate was used for phage tittering.

Eluted phages were used to infect $E$. coli to amplify them for the next rounds of panning. For this TG1 cells were pre-grown till OD600 $0.6-0.8$ in a $100 \mathrm{ml}$ culture at $37^{\circ} \mathrm{C}$. Prior to the infection the cell culture was kept still for 15 minutes to allow cell pili growth. After that the eluted phages as well as the helper phages were added to the culture and incubated for 30 minutes at $37^{\circ} \mathrm{C}$. At the end of the incubation time the culture was diluted with fresh $2 \mathrm{YT}$ medium with appropriate antibiotics. The culture was grown overnight at $37^{\circ} \mathrm{C}$ while shaking.

Typically four rounds of bio-panning were performed. Every next bio-panning round was with higher selection pressure to ensure that only specific and highly affine nanobodies are selected. To achieve this the number of washing steps was increased up to ten in the letter bio-panning steps.

\subsubsection{Re-cloning of bio-panned nanobodies into an expression vector}

In order to re-clone a nanobody library into an expression vector a DNA Midi had to be prepared. First, phages eluted during the last panning cycle were collected and used to infect a pre-grown TG1 E. coli cell culture. After $30 \mathrm{~min}$ incubation with no shaking 300 $\mathrm{ml}$ of 2 YT medium was added, supplemented with trimethoprim and grown over-night at $37^{\circ} \mathrm{C}$.

Then $50 \mathrm{ng}$ of phagemid was used for PCR reactions to amplify nanobodies and to introduce over-hangs for Gibson assembly. Alternatively Midi DNA preparation of the 
nanobody library was directly digested with restriction enzymes BamHF/NotI and cloned into a vector prepared in the same manner. Specifically, nanobodies were cloned either into pMG44 vector (His14-Biotin-tag-SumoStar-Nanobody) or into pMG141 (IM9-IF2SumoStar-sfGFP-Nanobody-His 10).

\subsubsection{Library sequencing and data analysis}

Typically 96 colonies were picked and grown in $300 \mu 1$ of 2YT supplemented with trimethoprim. These cultures were incubated in a 96-well plate in an incubator at $37^{\circ} \mathrm{C}$ under constant shaking for 6 hours. Approximately $150 \mu 1$ of each cell culture was transferred into a 96-well plate which was provided by Seqlab (Göttingen, Germany). The actual sequencing was done using the primer QE-rev (GTTCTGAGGTCATTACTGG). Sequencing retrieving and in silico translation was done using a Python script written by Dr. Koray Kirli. As a standard alignment tool MegAlign software was used and nanobodies with similar CDRIII loops were grouped into distinct classes. Usually 8-10 selected nanobodies were chosen for further expression and characterization.

\subsection{In vitro methods using Xenopus laevis egg extract}

\subsubsection{Interphase $X$. laevis egg extract}

Before egg extract preparation Xenopus laevis frogs were primed for ovulation for 3-14 days by subcutaneous injection of $50 \mathrm{U}$ human chorionic gonadotropin (hCG). One day prior egg collection frogs were injected with 500U of hCG and kept in separate containers with 1x Marc's Modified Ringers buffer (MMR). The next day laid eggs were inspected and any batches of bad quality (containing many apoptotic or activated eggs) were discarded. Batches of satisfactory quality were pulled together and washed with MMR buffer and the outer jelly layer of eggs was removed with $165 \mathrm{mM}$ L-cysteine $\mathrm{pH} 7.8$ in $0.25 \mathrm{x}$ MMR buffer for 5 minutes. In order to have all the eggs at the interphase state, they were treated with $80 \mu \mathrm{l}$ of calcium ionophore for $7 \mathrm{~min}$. Then, to remove the calcium ionophore the eggs were washed multiple times with 1x MMR buffer. Finally, they were packed into $13 \times 51 \mathrm{~mm}$ thin-wall centrifuge tubes (SETON Scientific, USA) and centrifuged for $30 \mathrm{~s}$ at $800 \mathrm{rpm}$ and consequently $90 \mathrm{~s}$ at $2000 \mathrm{rpm}$. Excess buffer was removed and the eggs were crushed by centrifugation for $20 \mathrm{~min}$ at $11000 \mathrm{rpm}$ at $4^{\circ} \mathrm{C}(\mathrm{RC} 6$ plus centrifuge, HB-6 rotor, Sorvall, USA). The resulting low-speed supernatant (LSS) was collected via the side puncture of the centrifugation tube with a syringe. The LSS was 
supplemented with $40 \mu \mathrm{g} / \mathrm{ml}$ cyclohexamide, $5 \mu \mathrm{g} / \mathrm{ml}$ cytochalasin $\mathrm{B}, 10 \mu \mathrm{M}$ caspase inhibitor Z-VAD-FMK (Calbiochem, USA), and a protease inhibitor. Upon addition of the chemicals mentioned above the LSS was frozen in $0.5 \mathrm{ml}$ aliquots in liquid nitrogen and stored at $-80^{\circ} \mathrm{C}$.

\subsubsection{Protein isolation from Xenopus laevis egg extract}

The interphase egg extract prepared as described above was diluted four-fold in S250 buffer (20 mM Hepes pH 7.5, 90 mM KAc, 2 mM MgAc, $250 \mathrm{mM}$ sucrose, 5 mM ATP, and $5 \mu \mathrm{g} / \mathrm{ml}$ cytochalasin B) and consequently centrifuged in $13 \times 51 \mathrm{~mm}$ thin-wall tubes (Seton Scientific, USA) for $1 \mathrm{~h}$ at $235000 \mathrm{~g}$ (Discovery M120 SE, S52ST, Sorvall, USA). The supernatant was collected by puncturing the tube side wall with a needle, this supernatant served as material for affinity purification.

The bait nanobodies were expressed as SUMOstar-fusions and biotinylated for immobilization onto magnetic Dynabeads MyOne Streptavidin T1 (Life Technologies, USA). Nanobodies were incubated with the magnetic beads for $1 \mathrm{~h}$ and non-bound material was discarded. The beads were washed with S250 buffer two times and then the interphase Xenopus laevis egg extract was added and incubated for $1 \mathrm{~h}$. Nanobody trapped protein complexes were washed several times with S250 buffer and eluted with SUMOstar protease for $1 \mathrm{~h}$ at $4^{\circ} \mathrm{C}$. Collected elution fractions were analyzed on SDS gels.

\subsubsection{In vitro assembled nuclei}

In vitro nuclear assembly was essentially done as described by Hülsmann et al. 2012 (and references therein). In short, $9 \mu 1 \mathrm{HSS}$ cytosol supplemented with $1 \mathrm{x}$ E-mix $(1 \mathrm{mM}$ HEPES/KOH pH 7.5, $10 \mathrm{mM}$ creatine phosphate, $0.5 \mathrm{mM}$ ATP, $0.5 \mathrm{mM} \mathrm{GTP}, 50 \mu \mathrm{g} / \mathrm{ml}$ creatine kinase, $12.5 \mathrm{mM}$ sucrose) were supplemented with $0.5 \mu 1$ of sperm chromatin and allowed to decondense for 10 minutes. Then the membranes were added $(1 \mu 1)$, and nuclear assembly proceeded for $2 \mathrm{~h}$ at $20^{\circ} \mathrm{C}$. After the formation of nuclei was finished, the nuclei were fixed by addition of $300 \mu 1$ of $2.4 \%$ paraformaldehyde in 1x PBS with $250 \mathrm{mM}$ sucrose for 15 minutes at room temperature. Nuclei prepared in this manner were centrifuged onto aminosilan-coated coverslips for $10 \mathrm{~min}$ at $3000 \mathrm{rpm}$ (Heraeus Multifuge, Thermo Scientific, USA). 
Lamin binding nanobodies were individually tested for their ability to bind Lamin B3 in in vitro assembled nuclei. Lamin B3 was visualized with fluorophore labeled and GFP-fused nanobodis. Essentially nanobodies were added either during nuclei assembly or to already fully assembled nuclei. In the first case nanobodies were incorporated during nuclei assembly by addition of $200 \mathrm{nM}$ of labeled nanobodies to HSS before sperm chromatin addition. In the second case $200 \mathrm{nM}$ nanobodies were added to already fully assembled nuclei and incubated for $30 \mathrm{~min}$ at $20^{\circ} \mathrm{C}$. DAPI was added in both experimental systems $(2 \mu \mathrm{g} / \mathrm{ml})$ in order to visualize DNA. For both options, stained nuclei were finally fixed in $500 \mu 1$ of $2,4 \%$ paraformaldehyde in $1 \mathrm{x}$ PBS for $15 \mathrm{~min}$. To test if fixation influences nanobodies ability to bind lamin samples were also first fixed and then treated with fluorescently labeled or GFP-fused nanobodies.

Treated nuclei were centrifuged onto aminosilan-coated coverslips (Heraeus Multifuge, Thermo Scientific, USA) for $10 \mathrm{~min}$ at $3000 \mathrm{rpm}$ at $20^{\circ} \mathrm{C}$ in $30 \%$ sucrose, $1 \mathrm{x}$ PBS in a $24-$ well plate. The coverslips were consequently washed twice with 1x PBS, desalted $\mathrm{H}_{2} \mathrm{O}$ and mounted with Vectashield (Vector Laboratories, Burlingame, USA). Samples were imaged on confocal microscopy.

\subsection{FG particles assays}

\subsubsection{Formation of FG repeat hydrogels}

Essentially FG repeat hydrogels were formed as reported (Frey and Gorlich, 2009; Frey and Görlich, 2007). In summary, lyophilized FG repeats were dissolved to the desired concentration in $0.2 \%$ TFA (in $\mathrm{ddH}_{2} \mathrm{O}$ ) containing 0.25-1 $\mu \mathrm{M}$ Alexa647-labeled MacNup98. One microliter drops of the re-suspended protein were placed onto 18-well collagen-coated micro slides (IBIDI, Martinsried, Germany) and allowed to gelate in humid environment at RT. If required the gelation process could be delayed by addition of 0.5-2 $\mathrm{M} \mathrm{GHCl}$ during re-suspension.

\subsubsection{FG repeats hydrogel permeation assay}

FG hydrogel was obtained by mixing recombinantly expressed MacNup98 with MacNup98-Alexa647 and supplemented with FG assay buffer (50 mM Tris pH 7.5, 130 $\mathrm{mM}, 2 \mathrm{mM} \mathrm{MgCl} 2,250 \mathrm{mM}$ sucrose, $2 \mathrm{mM}$ DTT) to $1 \mathrm{mM}$ final concentration. The concentration of labeled MacNup98 in the final solution was $25 \mu \mathrm{M}$ or $2.5 \%$. Next, the 
substrate molecules were mixed such that the concentration of the probe molecule was 0.7 $\mathrm{nM}$, the concentration of nuclear transport receptor was $1 \mu \mathrm{M}$, and finally mCherry served as a negative control $(8 \mu \mathrm{M})$. The cargo mixture was mixed and incubated for 1 hour at room temperature to ensure complex formation. At the end of the incubation time $8 \mu 1$ of cargo mixture were placed onto collagen coated micro slides (IBIDI, Martinsried, Germany). $20 \mu 1$ of previously pre-mixed FG particles were added to the cargo mixture and allowed to settle for 2 hours. The final concentrations of FG particles in the assay was $5 \mu \mathrm{M}$ (Frey and Gorlich, 2009; Frey and Görlich, 2007).

Import of fluorescent substrate molecules into FG repeat hydrogels was monitored using a confocal microscope Leica MZ6 with an 63x immersion objective (Mannheim, Germany). The $633 \mathrm{~nm}$ laser line was utilized to excite the tracer molecule MacNup98-Alexa647 and thus localize and adjust the position of FG particles. The $496 \mathrm{~nm}$ and $561 \mathrm{~nm}$ laser lines were used to excite the actual substrate of interest and the inert mCherry cargo respectively.

\subsection{Cell cultures and immunostaining}

\subsubsection{Transient transfection of cultured cells}

Lamin B3 containing vectors were prepared based on a modified pPEF2-GFP vector (Invitrogen, USA). Xenopus laevis XL177 epithelial cells (Ellison et al., 1985) as well as Homo sapiens Hela cell lines were used for transfection in this work. $1 \mathrm{x} 10^{4}$ cells were plated onto coverslips in a 24 well plate and left overnight to grow. Meanwhile a transfection mixture was prepared, which contains a plasmid DNA $(0.1 \mu \mathrm{g})$ and Lipofectamine 2000 (Thermofisher, USA). Cells were transfected upon reaching confluency of $50-80 \%$. After $24 \mathrm{~h}$ incubation time the cells were washed two times with $1 \mathrm{x}$ PBS buffer and quickly fixed by addition of $4 \%$ paraformaldehyde for 5 minutes. Any excess of paraformaldehyde was removed by several successive washes with 1x PBS. Fixed cells were stored up to a week until further use.

\subsubsection{Immunofluorescence}

Cultured cells were prepared as described in the "Transient transfection of cultured cells" section. Transfected and fixed cells were washed with 1x PBS and permeabilized with 0.3 $\%$ Triton X-100 in 1x PBS for 3-5 minutes. Consequently the cells were washed three 
times with 1x PBS to remove any excess of Triton X-100. In order to block any nonspecific background binding sites a 1\% BSA in 1x PBS solution was used to wash the cells for 30 minutes. Then a 100-200 nM concentration of fluorescently labeled nanobodies in 1x PBS was added to the cells and incubated for 45 minutes at RT, while gently shaking. After three washes with 1x PBS solution the coverslips were desalted in $\mathrm{ddH}_{2} \mathrm{O}$ and mounted on glass slides for confocal microscopy analysis.

\subsection{Structural methods}

\subsubsection{Cross-linking mass spectrometry for protein identification}

His-tagged anti-70S ribosome nanobodies and 70S ribosomes ( $1 \mathrm{nM}$ each) were incubated on ice for $1 \mathrm{~h}$ in $50 \mathrm{mM} \mathrm{K}_{2} \mathrm{HPO}_{4} \mathrm{pH} 6.8,300 \mathrm{mM} \mathrm{NaCl}$ to allow complex formation. Then a Mal-NHS cross-linking agent (Iris Biotech, Germany) was added in $2 \mathrm{nM}$ concentration and the $\mathrm{pH}$ was quickly raised to 7.5 by addition of $100 \mathrm{mM} \mathrm{K} \mathrm{HPO}_{4}$. The mixture was kept at $4^{\circ} \mathrm{C}$, while being gently agitated on a shaker. The reaction was stopped by addition of quenching reagents such as DTT or TCEP in 2-3 mM concentration.

In order to get rid of all the non cross-linked ribosomal proteins the samples were treated with SDS buffer. The SDS buffer causes denaturing of the ribosomal proteins and rRNA while the His-tagged nanobody retains the ability to bind $\mathrm{Ni}^{2+}$ resin. Thus, $50 \mu \mathrm{l}$ of $\mathrm{Ni}^{2+}$ magnetic beads were added to SDS treated samples and incubated for $1 \mathrm{~h}$ at $4^{\circ} \mathrm{C}$. Then, the samples were washed several times with $50 \mathrm{mM}$ Tris, $300 \mathrm{mM} \mathrm{NaCl}, 30 \mathrm{mM}$ imidazole buffer supplemented with SDS. Finally, samples were eluted with $50 \mu 1$ of $500 \mathrm{mM}$ imidazole in SDS buffer. One fifth of the eluate was analyzed by gel electrophoresis. A cross-linked nanobody to a ribosomal protein showed a mass shift. These bands were excised and analyzed by mass spectrometry. All mass spectrometry work on protein identification was carried out by Monika Raabe and Annika Kuehn.

\subsubsection{Nanobody epitope mapping by cross-linking}

Anti-Lamin B3 nanobodies pMG141, pMG143, and pMG152 and Lamin B3 full-length or Lamin B3 rod domain were first reduced with $5 \mathrm{mM}$ DTT. Then, the sample proteins were re-buffered on gel filtration colums PD-10 (GE Gealthcare Life Science, USA) into a buffer consisting of $50 \mathrm{mM} \mathrm{K}_{2} \mathrm{HPO}_{4} \mathrm{pH} 6.8,300 \mathrm{mM} \mathrm{NaCl}, 250 \mathrm{mM}$ sucrose. The samples were mixed in $\sim 16-20 \mu \mathrm{M}$ concentrations and a UVcross-linker was added. The UV cross- 
linker was activated for 2-10 minutes with $365 \mathrm{~nm}$ wavelength light. Next, the cross-linked samples were run on a SDS-PAGE for analysis and bands corresponding to cross-links were excised for in-gel digestion. Separated peptides were analyzed on Orbitrap Fusion mass spectrometer (Thermo Scientific, USA). The obtained data were searched against a FASTA database containing the sequences of respective nanobodies and Lamin B3 with pLink 1.22 software (Yang et al., 2012). 


\section{List of abbreviations}

\begin{tabular}{|c|c|}
\hline Amp & Ampicillin \\
\hline ATP & Adenosine 5'-triphosphate \\
\hline Avi-tag & Biotin acceptor peptide \\
\hline BSA & Bovine serum albumin \\
\hline C-terminus & Carboxy terminus \\
\hline CDR & Complementarity determining region \\
\hline $\mathrm{Cfu}$ & Colony forming unit \\
\hline ColE9 & Colicin E9 \\
\hline $\mathrm{COOH}$ & Carboxyl group \\
\hline DAPI & 4', 6'-diamidin-2-phenylindol \\
\hline DFHR & Dihydrofolate reductase \\
\hline DMF & Dimethylformamide \\
\hline DMSO & Dimethylsulfoxide \\
\hline DNA & Desoxyribonucleic acid \\
\hline DTT & Dithiothreitol \\
\hline E. coli & Escherichia coli \\
\hline EDTA & Ethylenediaminetetraacetic acid \\
\hline EM & Electron microscopy \\
\hline $\mathrm{Fab}$ & Antigen-binding fragment of an Ig molecule \\
\hline $\mathrm{Fc}$ & Crystallizable fragment of an Ig molecule \\
\hline FG & Phenylalanine-glycine dipeptide \\
\hline GA & Gibson assembly \\
\hline GFP & Green fluorescent protein \\
\hline HEPES & 4-(2-hydroxyethyl)-1-piperazineethanesulfonic acid \\
\hline IBB & Importin $\beta$-binding domain of Importin $\alpha$ \\
\hline IF2 & E. coli translation initation factor 2 \\
\hline iBAQ & Intensity based absolute quantification \\
\hline Ig & Immunoglobuline \\
\hline IM9 & Immunity protein 9 \\
\hline $\operatorname{Imp}$ & Importin \\
\hline IPTG & Isopropyl- $\beta$-D-thiogalactopyranosid \\
\hline $\mathrm{kDa}$ & Kilodalton \\
\hline LSS & Low speed supernatant \\
\hline $\mathrm{mAb}$ & Monoclonal antibody \\
\hline Mal & Maleimide \\
\hline MBP & Maltose binding protein \\
\hline MDa & Megadalton \\
\hline mRNA & Messenger RNA \\
\hline NPC & Nuclear pore complex \\
\hline $\mathrm{Nb}$ & Nanobody \\
\hline NH2 & Amino group \\
\hline NHS & N-Hydroxysuccinimide \\
\hline N-terminus & Amino-terminus \\
\hline NTR & Nuclear transport receptor \\
\hline Nup & Nucleoporin \\
\hline
\end{tabular}


OD600

Ori

pIII

PBS

PCR

PDB

PFA

PMSF

Ran

RanGAP

Ran-GTP

RanBP2

RNA

Rpm

RT

$\mathrm{scFv}$

SDS-PAGE

SH

SUMO

TB

Tth

TCEP

Tmp

Tris

$\mathrm{VH}$

VHH

VL

YT

Z-domain
Optical density measured at a wavelength of $600 \mathrm{~nm}$

Origin of replication

Bacteriophage M13KO7 minor coat protein III

Phosphate-buffered saline

Polymerase chain reaction

Protein data bank

Paraformaldehyde

Phenylmethanesulfonylfluoride

Ras-related nuclear protein

Ran GTPase-activating protein

GTP-bound Ran

Ran-binding protein 2

Ribonucleic acid

Revolutions per minute

Room temperature

Single-chain variable fragment

Sodium dodecyl sulfate polyacrylamide gel electrophoresis Thiol group

Small ubiquitin-related modifier

Terrific broth medium

Thermus thermophilus

Tris(2-carboxyethyl)phosphine

Trimethoprim

Tris(hydroxymethyl)aminomethane

Variable heavy chain domain of conventional antibodies

Variable heavy chain domain of heavy-chain-only antibodies

Variable light chain domain

Yeast extract tryptone medium

IgG-binding domain of Staphylococcus protein A 


\section{Acknowledgements}

First and foremost, I would like to express my gratitude to Prof. Görlich for teaching me how to do science and unveiling all the aspects of it. Thanks for all your help throughout my $\mathrm{PhD}$. I also would like to wish you to keep sharing your passion about science with younger generations for many years to come.

I also would like to thank my thesis committee members Prof. Bohnsack and Prof. Wienands for valuable comments, discussions, and criticism.

I would like to thank Prof. Bohnsack and his laboratory for the CRAC experiments. Prof. Urlaub and all his colleagues including Monika Raabe and Annika Kuehn for all the mass spectrometry work.

I would like to thank Sergey Trakhanov, for numerous practical advices and help with the preparation of ribosomes; Bastian Huelsmann for valuable help with the in vitro nuclei assambly, Volker Cordes, Tino Pleiner, Trevor Huyton, Sheung Chun Ng, Metin Aksu, Koray Kirli, and Kevser Fünfgeld for practical support and inspiring scientific conversations.

My deepest gratitude goes to Susanne Brandfass and Heinz-Juergen Dehne for your excellent technical support and help at different stages during my PhD.

I also would like to thank Gabriele Hawlitschek and Uwe Hoffmann for making our lab a great working place, we all greatly appreciate the work you do. Cornelia Paz is the greatest and most helpful department secretary, thanks a lot for all your work.

I would like to thank all the lab members for a pleasant working atmosphere.

I express my gratitude to all the members of the GGNB office for an excellent administrative support.

Arturo Vera, John Ng, Oleh Rymarenko, Cristin Kappert, and Mireia Sola are the people who are responsible for the most of my positive emotions I had during my working time. I would like to thank you altogether and each in person for creating a great atmosphere and your friendship that I will always value!

I thank my family for a continuous support during my studies and your trust in me. 
Finally, I would like to thank Ingrid who was always there for me. Whatever happens, I could always rely on you. I have a feeling that you could carry the whole world on your tender shoulders. Thank you for sharing my sorrow and happiness. Thank you for being my muse. 


\section{Curriculum vitae}

Name

Date of birth

Place of birth

Nationality
Myroslav Gebura

28.05.1989

Kalush, Ukraine

Ukrainian

\section{Education}

2013-2017 Doctoral studies in the Department of Cellular Logistics under the supervision of Prof. Dirk Goerlich at the Max Planck Institute for Biophysical chemistry, Goettingen, Germany

2012-2013 M. Sc. in Neurosciences and

Neuropsychopharmacology at the Bordeaux Sagalen University, France

2011-2013 M.Sc. in Neuroscience at the International Max Planck Research School (IMPRS) for Neuroscience at the Georg August University Goettingen, Germany

2006-2010

B. Sc. in Cell biology at Taras Shevchenko National University of Kyiv, Ukraine 


\section{References}

Aaronson, R.P., and Blobel, G. (1975). Isolation of nuclear pore complexes in association with a lamina. Proc. Natl. Acad. Sci. U. S. A. 72, 1007-1011.

Aebi, U., Cohn, J., Buhle, L., and Gerace, L. (1986). The nuclear lamina is a meshwork of intermediate-type filaments. Nature 323, 560-564.

Alberts, B., Johnson, A., Lewis, J., Raff, M., Roberts, K., and Walter, P. (2002). The Transport of Molecules between the Nucleus and the Cytosol.

Andersen, K.R., Onischenko, E., Tang, J.H., Kumar, P., Chen, J.Z., Ulrich, A., Liphardt, J.T., Weis, K., and Schwartz, T.U. (2013). Scaffold nucleoporins Nup188 and Nup192 share structural and functional properties with nuclear transport receptors. Elife 2013, 120.

von Appen, A., Kosinski, J., Sparks, L., Ori, A., DiGuilio, A.L., Vollmer, B., Mackmull, M.-T., Banterle, N., Parca, L., Kastritis, P., et al. (2015). In situ structural analysis of the human nuclear pore complex. Nature 526, 140-143.

Arenas, J.E., and Abelson, J.N. (1997). Prp43: An RNA helicase-like factor involved in spliceosome disassembly. Biochemistry 94, 11798-11802.

Bailer, S.M., Siniossoglou, S., Podtelejnikov, A., Hellwig, A., Mann, M., and Hurt, E. (1998). Nup116p and Nup100p are interchangeable through a conserved motif which constitutes a docking site for the mRNA transport factor Gle2p. EMBO J. 17, 1107-1119.

Ban, N., Nissen, P., Hansen, J., Moore, P.B., and Steitz, T.A. (2000). The complete atomic structure of the large ribosomal subunit at 2. Science (80-. ). 289, 905-920.

Bayliss, R., Ribbeck, K., Akin, D., Kent, H.M., Feldherr, C.M., Görlich, D., and Stewart, M. (1999). Interaction between NTF2 and xFxFG-containing nucleoporins is required to mediate nuclear import of RanGDP. J. Mol. Biol. 293, 579-593.

Beck, L.A., Hosick, T.J., and Sinensky, M. (1990). Isoprenylation is required for the processing of the lamin A precursor. J. Cell Biol. 110, 1489-1499.

Benavente, R., Krohne, G., and Franke, W.W. (1985). Cell type-specific expression of nuclear lamina proteins during development of Xenopus laevis. Cell 41, 177-190.

Bischoff, F.R., and Görlich, D. (1997). RanBP1 is crucial for the release of RanGTP from 
importin $\beta$-related nuclear transport factors. FEBS Lett. 419, 249-254.

Bischoff, F.R., and Ponstingl, H. (1995). Catalysis of guanine nucleotide exchange of Ran by RCC1 and stimulation of hydrolysis of Ran-bound GTP by Ran-GAP1. Methods Enzymol. 257, 135-144.

Bischoff, F.R., Krebber, H., Smirnova, E., Dong, W., and Ponstingl, H. (1995). Coactivation of RanGTPase and inhibition of GTP dissociation by Ran-GTP binding protein RanBP1. EMBO J. 14, 705-715.

Bjerke, S.L., and Roller, R.J. (2006). Roles for herpes simplex virus type 1 UL34 and US3 proteins in disrupting the nuclear lamina during herpes simplex virus type 1 egress. Virology 347, 261-276.

Blow, J.J., and Laskey, R.A. (1986). Initiation of DNA replication in nuclei and purified DNA by a cell-free extract of Xenopus eggs. Cell 47, 577-587.

Bohnsack, M.T., Martin, R., Granneman, S., Ruprecht, M., Schleiff, E., and Tollervey, D. (2009). Prp43 Bound at Different Sites on the Pre-rRNA Performs Distinct Functions in Ribosome Synthesis. Mol. Cell 36, 583-592.

Bohnsack, M.T., Tollervey, D., and Granneman, S. (2012). Identification of RNA helicase target sites by UV cross-linking and analysis of cDNA. In Methods in Enzymology, pp. $275-288$.

Bollati, M., Barbiroli, A., Favalli, V., Arbustini, E., Charron, P., and Bolognesi, M. (2012). Structures of the lamin A/C R335W and E347K mutants: Implications for dilated cardiolaminopathies. Biochem. Biophys. Res. Commun. 418, 217-221.

Bonner, W.M. (1975). Protein migration into nuclei: I. frog oocyte nuclei in vivo accumulate microinjected histones, allow entry to small proteins, and exclude large proteins. J. Cell Biol. 64, 421-430.

Branston, S.D., Stanley, E.C., Ward, J.M., and Keshavarz-Moore, E. (2013). Determination of the Survival of Bacteriophage M13 from Chemical and Physical Challenges to Assist in Its Sustainable Bioprocessing. Biotechnol. Bioprocess Eng. 18, $560-566$.

Breitling, F., Dübel, S., Seehaus, T., Klewinghaus, I., and Little, M. (1991). A surface expression vector for antibody screening. Gene 104, 147-153. 
Brimacombe, R., Stiege, W., Kyriatsoulis, A., and Maly, P. (1988). Intra-RNA and RNAprotein cross-linking techniques in Escherichia coli ribosomes. pp. 287-309.

Broers, J.L. V, Bronnenberg, N.M.H.J., Kuijpers, H.J.H., Schutte, B., Hutchison, C.J., and Ramaekers, F.C.S. (2002). Partial cleavage of A-type lamins concurs with their total disintegration from the nuclear lamina during apoptosis.

Brownawell, A.M., and Macara, I.G. (2002). Exportin-5, a novel karyopherin, mediates nuclear export of double-stranded RNA binding proteins. J. Cell Biol. 5312, 21-9525.

Burke, B. (1990). On the Cell-Free Association with Metaphase Chromosomes. Exp. Cell Res. 186, 169-176.

Chelsky, D., Olson, J.F., and Koshland, D.E. (1987). Cell cycle-dependent methyl esterification of lamin B. J. Biol. Chem. 262, 4303-4309.

Chen, Z.A., Jawhari, A., Fischer, L., Buchen, C., Tahir, S., Kamenski, T., Rasmussen, M., Lariviere, L., Bukowski-Wills, J.-C., Nilges, M., et al. (2010). Architecture of the RNA polymerase II-TFIIF complex revealed by cross-linking and mass spectrometry. EMBO J. $29,717-726$.

Chomczynski, P., and Sacchi, N. (1987). Single-step method of RNA isolation by acid guanidinium thiocyanate-phenol-chloroform extraction. Anal. Biochem. 162, 156-159.

Chook, Y.M., and Blobel, G. (1999). Structure of the nuclear transport complex karyopherin-beta2-Ran x GppNHp. Nature 399, 230-237.

Chug, H., Trakhanov, S., and Pleiner, T. (2014). Crystal structure of the metazoan Nup62 • Nup58 • Nup54 nucleoporin complex. Science (80-. ). 250, 106-110.

Combe, C.W., Fischer, L., and Rappsilber, J. (2015). xiNET: cross-link network maps with residue resolution. Mol. Cell. Proteomics 14, mcp.O114.042259.

Conti, E., Müller, C.W., and Stewart, M. (2006). Karyopherin flexibility in nucleocytoplasmic transport. Curr. Opin. Struct. Biol. 16, 237-244.

Cremer, T., Cremer, M., Dietzel, S., Müller, S., Solovei, I., and Fakan, S. (2006). Chromosome territories - a functional nuclear landscape. Curr. Opin. Cell Biol. 18, 307316.

Croft, J.A., Bridger, J.M., Boyle, S., Perry, P., Teague, P., and Bickmore, W.A. (1999). 
Differences in the localization and morphology of chromosomes in the human nucleus. J. Cell Biol. 145, 1119-1131.

Day, L.A., and Wiseman, R.L. (1978). A Comparison of DNA Packaging in the Virions of fd , Xf , and Pf1.605-625.

Dechat, T., Gajewski, A., Korbei, B., Gerlich, D., Daigle, N., Haraguchi, T., Furukawa, K., Ellenberg, J., and Foisner, R. (2004). LAP2alpha and BAF transiently localize to telomeres and specific regions on chromatin during nuclear assembly. J. Cell Sci. 117, 6117-6128.

Dechat, T., Pfleghaar, K., Sengupta, K., Shimi, T., Shumaker, D.K., Solimando, L., and Goldman, R.D. (2008). Nuclear Lamins, Major Factors in the Structual Organization and Function of the Nucleus and Chromain.Pdf. Genes Dev 22, 832-853.

Delbarre, E., Tramier, M., Coppey-Moisan, M., Gaillard, C., Courvalin, J.C., and Buendia, B. (2006). The truncated prelamin A in Hutchinson-Gilford progeria syndrome alters segregation of A-type and B-type lamin homopolymers. Hum. Mol. Genet. 15, 1113-1122.

Denning, D.P., Patel, S.S., Uversky, V., Fink, A.L., and Rexach, M. (2003). Disorder in the nuclear pore complex: The FG repeat regions of nucleoporins are natively unfolded. Proc. Natl. Acad. Sci. 100, 2450-2455.

Dhe-Paganon, S., Werner, E.D., Chi, Y.I., and Shoelson, S.E. (2002). Structure of the globular tail of nuclear lamin. J. Biol. Chem. 277, 17381-17384.

Diaconu, M., Kothe, U., Schlünzen, F., Fischer, N., Harms, J.M., Tonevitsky, A.G., Stark, H., Rodnina, M. V., and Wahl, M.C. (2005). Structural basis for the function of the ribosomal L7/12 stalk in factor binding and GTpase activation. Cell 121,991-1004.

Dingwall, C., and Laskey, R.A. (1991). Nuclear targeting sequences - a consensus? Amino Acids 16, 478-481.

Dolk, E., van der Vaart, M., Lutje Hulsik, D., Vriend, G., de Haard, H., Spinelli, S., Cambillau, C., Frenken, L., and Verrips, T. (2005). Isolation of llama antibody fragments for prevention of dandruff by phage display in shampoo. Appl. Environ. Microbiol. 71, $442-450$.

Dong, L., Lv, L.-B., and Lai, R. (2012). [Molecular cloning of Tupaia belangeri chinensis neuropeptide $\mathrm{Y}$ and homology comparison with other analogues from primates]. (Cold Spring Harbor, NY, USA: Cold Spring Harbor Laboratory Press). 
Dudek, S.M., Chiang, E.T., Camp, S.M., Guo, Y., Zhao, J., Brown, M.E., Singleton, P. a, Wang, L., Desai, A., Arce, F.T., et al. (2010). Abl tyrosine kinase phosphorylates nonmuscle Myosin light chain kinase to regulate endothelial barrier function. Mol. Biol. Cell 21, 4042-4056.

Dumoulin, M., Conrath, K., Van Meirhaeghe, A., Meersman, F., Heremans, K., Frenken, L.G.J., Muyldermans, S., Wyns, L., and Matagne, A. (2002). Single-domain antibody fragments with high conformational stability. Protein Sci. 11, 500-515.

Dwyer, N. (1976). NANCY DWYER and GISNTER BLOBEL From The Rockefeller University, New York 10021. J. Cell Biol. 70, 581-591.

EGGERT, M., RADOMSKI, N., LINDER, D., TRIPIER, D., TRAUB, P., and JOST, E. (1993). Identification of novel phosphorylation sites in murine A-type lamins. Eur. J. Biochem. 213, 659-671.

Eibauer, M., Pellanda, M., Turgay, Y., Dubrovsky, A., Wild, A., and Medalia, O. (2015). Structure and gating of the nuclear pore complex. Nat. Commun. 6, 7532.

Ellis, D.J., Jenkins, H., Whitfield, W.G., and Hutchison, C.J. (1997). GST-lamin fusion proteins act as dominant negative mutants in Xenopus egg extract and reveal the function of the lamina in DNA replication. J. Cell Sci. 110 ( Pt 2, 2507-2518.

Ellison, T.R., Mathisen, P.M., and Miller, L. (1985). Developmental changes in keratin patterns during epidermal maturation. Dev. Biol. 112, 329-337.

Englmeier, L., Olivo, J.C., and Mattaj, I.W. (1999). Receptor-mediated substrate translocation through the nuclear pore complex without nucleotide triphosphate hydrolysis. Curr. Biol. 9, 30-41.

Farnsworth, C.C., Wolda, S.L., Gelb, M.H., and Howard, J.A.G. (1987). Human Lamin B Contains a Farnesylated Cysteine Residue*. J Biol Chem.

Firmbach-Kraft, I., and Stick, R. (1993). The role of CaaX-dependent modifications in membrane association of Xenopus nuclear lamin B3 during meiosis and the fate of B3 in transfected mitotic cells. J. Cell Biol. 123, 1661-1670.

Fischer, U., Huber, J., Boelens, W.C., Mattajt, L.W., and Lührmann, R. (1995). The HIV-1 Rev Activation Domain is a nuclear export signal that accesses an export pathway used by specific cellular RNAs. Cell 82, 475-483. 
Forbes, D.J., Kirschner, M.W., and Newport, J.W. (1983). Spontaneous formation of nucleus-like structures around bacteriophage DNA microinjected into Xenopus eggs. Cell $34,13-23$.

Fornerod, M., Van Deursen, J., Van Baal, S., Reynolds, A., Davis, D., Murti, K.G., Fransen, J., and Grosveld, G. (1997a). The human homologue of yeast CRM1 is in a dynamic subcomplex with CAN/Nup214 and a novel nuclear pore component Nup88. EMBO J. 16, 807-816.

Fornerod, M., Ohno, M., Yoshida, M., and Mattaj, I.W. (1997b). CRM1 Is an Export Receptor for Leucine-Rich Nuclear Export Signals. Cell 90, 1051-1060.

Frey, S., and Gorlich, D. (2009). FG/FxFG as well as GLFG repeats form a selective permeability barrier with self-healing properties. Embo J. 28, 2554-2567.

Frey, S., and Görlich, D. (2007). A Saturated FG-Repeat Hydrogel Can Reproduce the Permeability Properties of Nuclear Pore Complexes. Cell 130, 512-523.

Frey, S., and Görlich, D. (2014). Purification of protein complexes of defined subunit stoichiometry using a set of orthogonal, tag-cleaving proteases. J. Chromatogr. A 1337, $106-115$.

Frey, S., Richter, R.P., and Gorlich, D. (2006). FG-Rich Repeats of Nuclear Pore Proteins Form a Three-Dimensional Meshwork with Hydrogel-Like Properties. Science (80-. ). 314, $815-817$.

Gadal, O., Strauss, D., Kessl, J., Trumpower, B., Tollervey, D., and Hurt, E. (2001). Nuclear export of 60s ribosomal subunits depends on Xpo1p and requires a nuclear export sequence-containing factor, $\mathrm{Nmd} 3 \mathrm{p}$, that associates with the large subunit protein Rpl10p. Mol. Cell. Biol. 21, 3405-3415.

Gallan H.G., T.S.. (1950). Experimental studies on amphibian oocyte nuclei. I. Investigation of the structure of the nuclear membrane by means of the electron microscope. Proc R Soc L. B Biol Sci.

De Genst, E., Silence, K., Decanniere, K., Conrath, K., Loris, R., Kinne, J., Muyldermans, S., and Wyns, L. (2006). Molecular basis for the preferential cleft recognition by dromedary heavy-chain antibodies. Proc. Natl. Acad. Sci. U. S. A. 103, 4586-4591.

Gerace, L., and Blobel, G. (1980). The nuclear envelope lamina is reversibly 
depolymerized during mitosis. Cell 19, 277-287.

Gerace, L., Blum, A., and Blobel, G. (1978). Immunocytochemical localization of the major polypeptides of the nuclear pore complex-lamina fraction: Interphase and mitotic distribution. J. Cell Biol. 79, 546-566.

Gibson, D.G. (2011). Enzymatic assembly of overlapping DNA fragments. Methods Enzymol. 498, 349-361.

Gieffers, C., and Krohne, G. (1991). In vitro reconstitution of recombinant lamin A and a lamin A mutant lacking the carboxy-terminal tail. Eur J Cell Biol 55, 191-199.

Goldberg, M.W., Huttenlauch, I., Hutchison, C.J., and Stick, R. (2008). Filaments made from A- and B-type lamins differ in structure and organization. J. Cell Sci. 121, 215-225.

Görlich, D., and Kutay, U. (1999). Transport Between the Cell Nucleus and the Cytoplasm. Annu. Rev. Cell Dev. Biol. 15, 607-660.

Görlich, D., Prehn, S., Laskey, R.A., and Hartmann, E. (1994). Isolation of a protein that is essential for the first step of nuclear protein import. Cell 79, 767-778.

Görlich, D., Panté, N., Kutay, U., Aebi, U., and Bischoff, F.R. (1996). Identification of different roles for RanGDP and RanGTP in nuclear protein import. EMBO J. 15, 55845594.

Görlich, D., Dabrowski, M., Bischoff, F.R., Kutay, U., Bork, P., Hartmann, E., Prehn, S., and Izaurralde, E. (1997). A novel class of RanGTP binding proteins. J. Cell Biol. 138, 6580 .

Görlich, D., Seewald, M.J., and Ribbeck, K. (2003). Characterization of Ran-driven cargo transport and the RanGTPase system by kinetic measurements and computer simulation. EMBO J. 22, 1088-1100.

Groves, M.R., Hanlon, N., Turowski, P., Hemmings, B.A., and Barford, D. (1999). The structure of the protein phosphatase 2A PR65/A subunit reveals the conformation of its 15 tandemly repeated HEAT motifs. Cell 96, 99-110.

Gruenbaum, Y., Landesman, Y., Drees, B., Bare, J.W., Saumweber, H., Paddy, M.R., Sedat, J.W., Smith, D.E., Benton, B.M., and Fisher, P.A. (1988). Drosophila nuclear lamin precursor Dm0 is translated from either of two developmentally regulated mRNA species 
apparently encoded by a single gene. J. Cell Biol. 106, 585-596.

Güttler, T., Madl, T., Neumann, P., Deichsel, D., Corsini, L., Monecke, T., Ficner, R., Sattler, M., and Görlich, D. (2010). NES consensus redefined by structures of PKI-type and Rev-type nuclear export signals bound to CRM1. Nat. Struct. Mol. Biol. 17, 13671376.

H. B. Schmidt, D.G. (2015). Nup98 FG domains from diverse species spontaneously phase-separate into particles with nuclear pore-like permselectivity. Elife.

Hansen, C.B., Kao, G.Y., Moase, E.H., Zalipsky, S., and Allen, T.M. (1995). Attachment of antibodies to sterically stabilized liposomes: evaluation, comparison and optimization of coupling procedures. BBA - Biomembr. 1239, 133-144.

Hawlisch, H., Müller, M., Frank, R., Bautsch, W., Klos, A., and Köhl, J. (2001). SiteSpecific Anti-C3a Receptor Single-Chain Antibodies Selected by Differential Panning on Cellulose Sheets. Anal. Biochem. 293, 142-145.

Heald, R., and McKeon, F. (1990). Mutations of phosphorylation sites in lamin A that prevent nuclear lamina disassembly in mitosis. Cell 61, 579-589.

Ho, J.H.-N., Kallstrom, G., and Johnson, A.W. (2000). Nmd3p Is a Crm1p-Dependent Adapter Protein for Nuclear Export of the Large Ribosomal Subunit. J. Cell Biol. 151, 1057-1066.

Hockensmith, J.W., Kubasek, W.L., Vorachek, W.R., and von Hippel, P.H. (1986). Laser cross-linking of nucleic acids to proteins. Methodology and first applications to the phage T4 DNA replication system. J. Biol. Chem. 261, 3512-3518.

Hülsmann, B.B., Labokha, A.A., and Görlich, D. (2012). The permeability of reconstituted nuclear pores provides direct evidence for the selective phase model. Cell 150, 738-751.

Hurt, E.C. (1988). A novel nucleoskeletal-like protein located at the nuclear periphery is required for the life cycle of Saccharomyces cerevisiae. EMBO J. 7, 4323-4334.

Hurt, E., Hannus, S., Schmelzl, B., Lau, D., Tollervey, D., and Simos, G. (1999). A novel in vivo assay reveals inhibition of ribosomal nuclear export in Ran-cycle and nucleoporin mutants. J. Cell Biol. 144, 389-401.

Hust, M., Maiss, E., Jacobsen, H.J., and Reinard, T. (2002). The production of a genus- 
specific recombinant antibody ( $\mathrm{scFv}$ ) using a recombinant potyvirus protease. J. Virol. Methods 106, 225-233.

Iovine, M.K., Watkins, J.L., and Wente, S.R. (1995). The GLFG repetitive region of the nucleoporin Nup116p interacts with Kap95p, an essential yeast nuclear import factor. J. Cell Biol. 131, 1699-1713.

Isobe, K., Gohara, R., Ueda, T., Takasaki, Y., and Ando, S. (2007). The Last Twenty Residues in the Head Domain of Mouse Lamin A Contain Important Structural Elements for Formation of Head-to-Tail Polymers in Vitro. Biosci. Biotechnol. Biochem. 71, 12521259.

Jäkel, S., and Görlich, D. (1998). Importin $\beta$, transportin, RanBP5 and RanBP7 mediate nuclear import of ribosomal proteins in mammalian cells. EMBO J. 17, 4491-4502.

Johnson, B.R., Nitta, R.T., Frock, R.L., Mounkes, L., Barbie, D.A., Stewart, C.L., Harlow, E., and Kennedy, B.K. (2004). A-type lamins regulate retinoblastoma protein function by promoting subnuclear localization and preventing proteasomal degradation. Proc. Natl. Acad. Sci. 101, 9677-9682.

Kalderon, D., Richardson, W.D., Markham, a F., and Smith, a E. (1984). Sequence requirements for nuclear location of simian virus 40 large-T antigen. Nature 311, 33-38.

Karabinos, A., Schünemann, J., Meyer, M., Aebi, U., and Weber, K. (2003). The single nuclear lamin of Caenorhabditis elegans forms in vitro stable intermediate filaments and paracrystals with a reduced axial periodicity. J. Mol. Biol. 325, 241-247.

Kehlenbach, R.H. (1954). Pores in mammalian nucmlear membrane.

Klapper, M., Exner, K., Kempf, A., Gehrig, C., Stuurman, N., Fisher, P.A., Krohne, G., Krohne, G., Stuurman, N., and Kempf, A. (1997). Authors' correction Assembly of A-and B-type lamins studied in vivo with the baculovirus system. J. Cell Sci. 110, 2519-2532.

Korostelev, A., Trakhanov, S., Laurberg, M., and Noller, H.F. (2006a). Crystal Structure of a 70S Ribosome-tRNA Complex Reveals Functional Interactions and Rearrangements. Cell 126, 1065-1077.

Korostelev, A., Trakhanov, S., Laurberg, M., and Noller, H.F. (2006b). Crystal Structure of a 70S Ribosome-tRNA Complex Reveals Functional Interactions and Rearrangements. Cell 126, 1065-1077. 
Kumaran, R.I., Muralikrishna, B., and Parnaik, V.K. (2002). Lamin A/C speckles mediate spatial organization of splicing factor compartments and RNA polymerase II transcription. J. Cell Biol. 159, 783-793.

Kutay, U., Ralf Bischoff, F., Kostka, S., Kraft, R., and Görlich, D. (1997). Export of importin $\alpha$ from the nucleus is mediated by a specific nuclear transport factor. Cell 90 , 1061-1071.

Laemmli, U.K. (1970). Cleavage of structural proteins during the assembly of the head of bacteriophage T4. Nature 227, 680-685.

Lasa, I., Castón, J.R., Fernández-Herrero, L.A., de Pedro, M.A., and Berenguer, J. (1992). Insertional mutagenesis in the extreme thermophilic eubacteria Thermus thermophilus HB8. Mol. Microbiol. 6, 1555-1564.

Lawyer, F.C., Stoffel, S., Saiki, R.K., Chang, S.Y., Landre, P.A., Abramson, R.D., and Gelfand, D.H. (1993). High-level expression, purification, and enzymatic characterization of full-length Thermus aquaticus DNA polymerase and a truncated form deficient in $5^{\prime}$ to 3' exonuclease activity. Genome Res. 2, 275-287.

Lee, C.-P., Huang, Y.-H., Lin, S.-F., Chang, Y., Chang, Y.-H., Takada, K., and Chen, M.R. (2008). Epstein-Barr Virus BGLF4 Kinase Induces Disassembly of the Nuclear Lamina To Facilitate Virion Production. J. Virol. 82, 11913-11926.

Lehner, C.F., Fürstenberger, G., Eppenberger, H.M., and Nigg, E.A. (1986). Biogenesis of the nuclear lamina: in vivo synthesis and processing of nuclear protein precursors. Proc. Natl. Acad. Sci. U. S. A. 83, 2096-2099.

Liu, W., Xie, Y., Ma, J., Luo, X., Nie, P., Zuo, Z., Lahrmann, U., Zhao, Q., Zheng, Y., Zhao, Y., et al. (2015). IBS: An illustrator for the presentation and visualization of biological sequences. Bioinformatics 31, 3359-3361.

Loewinger, L., and Mckeon, F. (1988). Mutations in the nuclear lamin proteins resulting in their aberrant assembly in the cytoplasm. EMBO J. 7, 2301-2309.

Lohka, M., and Masui, Y. (1983). Formation in vitro of sperm pronuclei and mitotic chromosomes induced by amphibian ooplasmic components. Science (80-. ). 220, 719721. 
Lourim, D., and Krohne, G. (1998). Chromatin binding and polymerization of the endogenous Xenopus egg lamins: the opposing effects of glycogen and ATP. J. Cell Sci. 111 ( Pt 2, 3675-3686.

Lourim, D., Kempf, a, and Krohne, G. (1996). Characterization and quantitation of three B-type lamins in Xenopus oocytes and eggs: increase of lamin LI protein synthesis during meiotic maturation. J. Cell Sci. 109 ( Pt 7, 1775-1785.

Lowman, H.B., and Clackson, T. (2004). Phage Display: A Practical Approach (New York: Oxford University Press).

Ma, C., Wu, S., Li, N., Chen, Y., Yan, K., Li, Z., Zheng, L., Lei, J., Woolford, J.L., and Gao, N. (2017). Structural snapshot of cytoplasmic pre-60S ribosomal particles bound by Nmd3, Lsg1, Tif6 and Reh1. Nat. Struct. Mol. Biol. 24, 214-220.

Maass, D.R., Sepulveda, J., Pernthaner, A., and Shoemaker, C.B. (2007). Alpaca (Lama pacos) as a convenient source of recombinant camelid heavy chain antibodies (VHHs). J. Immunol. Methods 324, 13-25.

Malhas, A., Lee, C.F., Sanders, R., Saunders, N.J., and Vaux, D.J. (2007). Defects in lamin B1 expression or processing affect interphase chromosome position and gene expression. $\mathrm{J}$. Cell Biol. 176, 593-603.

Mancini, M.A., Shan, B., Nickerson, J.A., Penman, S., and Lee, W.H. (1994). The retinoblastoma gene product is a cell cycle-dependent, nuclear matrix-associated protein. Proc. Natl. Acad. Sci. U. S. A. 91, 418-422.

Markiewicz, E. (2002). Lamin A/C Binding Protein LAP2alpha Is Required for Nuclear Anchorage of Retinoblastoma Protein. Mol. Biol. Cell 13, 4401-4413.

Martineau, P. (2010). Antibody Engineering (Totowa, NJ: Humana Press).

McConnell, S.J., Dinh, T., Le, M.H., and Spinella, D.G. (1999). Biopanning phage display libraries using magnetic beads vs. polystyrene plates. Biotechniques 26, 208-214.

Mingot, J.M., Kostka, S., Kraft, R., Hartmann, E., and Görlich, D. (2001). Importin 13: A novel mediator of nuclear import and export. EMBO J. 20, 3685-3694.

von Moeller, F., Barendziak, T., Apte, K., Goldberg, M.W., and Stick, R. (2010). Molecular characterization of Xenopus lamin LIV reveals differences in the lamin 
composition of sperms in amphibians and mammals. Nucleus 1,85-95.

Moir, R.D., Donaldson, A.D., and Stewart, M. (1991). Expression in Escherichia coli of human lamins $\mathrm{A}$ and $\mathrm{C}$ : influence of head and tail domains on assembly properties and paracrystal formation. J. Cell Sci. 99 ( Pt 2), 363-372.

Moir, R.D., Yoon, M., Khuon, S., and Goldman, R.D. (2000). Nuclear lamins A and B1: Different pathways of assembly during nuclear envelope formation in living cells. J. Cell Biol. 151, 1155-1168.

Monecke, T., Haselbach, D., Voß, B., Russek, A., Neumann, P., Thomson, E., Hurt, E., Zachariae, U., Stark, H., Grubmüller, H., et al. (2013). Structural basis for cooperativity of CRM1 export complex formation. Proc. Natl. Acad. Sci. U. S. A. 110, 960-965.

Moy, T.I., and Silver, P.A. (2002). Requirements for the nuclear export of the small ribosomal subunit. J. Cell Sci. 115, 2985-2995.

Noller, H.F., Hoffarth, V., and Zimniak, L. (1992). Unusual Resistance of Peptidyl Transferase to Protein Extraction Procedures. Source Sci. New Ser. Transplant. Bull. Ann. Surg. Br. Med. J 256, 1416-1419.

Noppe, W., Plieva, F., Galaev, I.Y., Pottel, H., Deckmyn, H., and Mattiasson, B. (2009). Chromato-panning: an efficient new mode of identifying suitable ligands from phage display libraries. BMC Biotechnol. 9, 21.

Okonechnikov, K., Golosova, O., and Fursov, M. (2012). Unipro UGENE: a unified bioinformatics toolkit. Bioinforma. Appl. NOTE 28, 1166-116710.

Ori, A., Banterle, N., Iskar, M., Andres-Pons, A., Escher, C., Khanh Bui, H., Sparks, L., Solis-Mezarino, V., Rinner, O., Bork, P., et al. (2013). Cell type-specific nuclear pores: a case in point for context-dependent stoichiometry of molecular machines. Mol. Syst. Biol. 9, 648-648.

Oshima, T., and Imahori, K. (1974). Description of Thermus thermophilus (Yoshida and Oshima) comb. nov., a Nonsporulating Thermophilic Bacterium from a Japanese Thermal Spa. Int. J. Syst. Bacteriol. 24, 102-112.

Pajerowski, J.D., Dahl, K.N., Zhong, F.L., Sammak, P.J., and Discher, D.E. (2007). Physical plasticity of the nucleus in stem cell differentiation. Proc. Natl. Acad. Sci. 104, $15619-15624$. 
Pashev, I.G., Dimitrov, S.I., and Angelov, D. (1991). Crosslinking proteins to nucleic acids by ultraviolet laser irradiation. Tibs $16,323-326$.

Peter, A., and Stick, R. (2012). Evolution of the lamin protein family. Nucleus 3, 44-59.

Petosa, C., Schoehn, G., Askjaer, P., Bauer, U., Moulin, M., Steuerwald, U., Soler-López, M., Baudin, F., Mattaj, I.W., and Müller, C.W. (2004). Architecture of CRM1/Exportin1 suggests how cooperativity is achieved during formation of a nuclear export complex. Mol. Cell 16,761-775.

Pleiner, T., Bates, M., Trakhanov, S., Lee, C.T., Schliep, J.E., Chug, H., Böhning, M., Stark, H., Urlaub, H., and Görlich, D. (2015). Nanobodies: Site-specific labeling for superresolution imaging, rapid epitope- mapping and native protein complex isolation. Elife 4, $1-21$.

Reichelt, R., Holzenburg, A., Buhle, E.L., Jarnik, M., Engel, A., and Aebi, U. (1990). Correlation between structure and mass distribution of the nuclear pore complex and of distinct pore complex components. J. Cell Biol. 110, 883-894.

Ribbeck, K., and Görlich, D. (2001). Kinetic analysis of translocation through nuclear pore complexes. EMBO J. 20, 1320-1330.

Ribbeck, K., and Görlich, D. (2002). The permeability barrier of nuclear pore complexes appears to operate via hydrophobic exclusion. EMBO J. 21, 2664-2671.

Ribbeck, K., Lipowsky, G., Kent, H.M., Stewart, M., and Görlich, D. (1998). NTF2 mediates nuclear import of Ran. EMBO J. 17, 6587-6598.

Riemer, D., Dodemont, H., and Weber, K. (1993). A nuclear lamin of the nematode Caenorhabditis elegans with unusual structural features; cDNA cloning and gene organization. Eur J Cell Biol 62, 214-223.

Rothbauer, U., Zolghadr, K., Muyldermans, S., Schepers, A., Cardoso, M.C., and Leonhardt, H. (2007). A Versatile Nanotrap for Biochemical and Functional Studies with Fluorescent Fusion Proteins. Mol. Cell. Proteomics 7, 282-289.

Rout, M.P., and Blobel, G. (1993). Isolation of the yeast nuclear pore complex. J. Cell Biol. 123, 771-783.

Rout, M.P., Aitchison, J.D., Suprapto, A., Hjertaas, K., Zhao, Y., and Chait, B.T. (2000). 
The yeast nuclear pore complex: Composition, architecture, transport mechanism. J. Cell Biol. 148, 635-651.

Santos-Rosa, H., Moreno, H., Simos, G., Segref, A., Fahrenkrog, B., Panté, N., and Hurt, E. (1998). Nuclear mRNA export requires complex formation between Mex67p and Mtr2p at the nuclear pores. Mol. Cell. Biol. 18, 6826-6838.

Schatz, P.J. (1993). Use of peptide libraries to map the substrate specificity of a peptidemodifying enzyme: a 13 residue consensus peptide specifies biotinylation in Escherichia coli. Biotechnology. (N. Y). 11, 1138-1143.

Schirmer, E.C., Guan, T., and Gerace, L. (2001). Involvement of the lamin rod domain in heterotypic lamin interactions important for nuclear organization. J. Cell Biol. 152, 479489.

Schmidt, H.B., and Görlich, D. (2015). Nup98 FG domains from diverse species spontaneously phase-separate into particles with nuclear pore-like permselectivity. Elife 4 .

Schwartz, T.U. (2005). Modularity within the architecture of the nuclear pore complex. Curr. Opin. Struct. Biol. 15, 221-226.

Sedelnikova, S.E., Agalarov, S.C., Garber, M.B., and Yusupov, M.M. (1987). Proteins of the Thermus thermophilus ribosome Purification of several individual proteins and crystallization of protein TL7. FEBS Lett. 220, 227-230.

Shimi, T., Pfleghaar, K., Kojima, S.I., Pack, C.G., Solovei, I., Goldman, A.E., Adam, S.A., Shumaker, D.K., Kinjo, M., Cremer, T., et al. (2008). The A- and B-type nuclear lamin networks: Microdomains involved in chromatin organization and transcription. Genes Dev. $22,3409-3421$.

Shumaker, D.K., Lopez-Soler, R.I., Adam, S.A., Herrmann, H., Moir, R.D., Spann, T.P., and Goldman, R.D. (2005). Functions and dysfunctions of the nuclear lamin Ig-fold domain in nuclear assembly, growth, and Emery-Dreifuss muscular dystrophy. Proc. Natl. Acad. Sci. U. S. A. 102, 15494-15499.

Smith, G.P. (1985). Filamentous fusion phage: novel expression vectors that display cloned antigens on the virion surface. Science 228, 1315-1317.

Sørensen, H.P., Sperling-Petersen, H.U., and Mortensen, K.K. (2003). A favorable solubility partner for the recombinant expression of streptavidin. Protein Expr. Purif. 32, 
Spann, T.P., Moir, R.D., Goldman, A.E., Stick, R., and Goldman, R.D. (1997). Disruption of nuclear lamin organization alters the distribution of replication factors and inhibits DNA synthesis. J. Cell Biol. 136, 1201-1212.

Stage-Zimmermann, T., Schmidt, U., and Silver, P.A. (2000). Factors affecting nuclear export of the 60S ribosomal subunit in vivo. Mol. Biol. Cell 11,3777-3789.

Steiner, D., Forrer, P., and Plückthun, A. (2008). Efficient Selection of DARPins with Subnanomolar Affinities using SRP Phage Display. J. Mol. Biol. 382, 1211-1227.

Stick, R. (1992). The Gene Structure of Xenopus Nuclear Lamin A: A Model for the Evolution of A-type from B-type Lamins by Exon Shuffling. Chromosoma 101, 566-574.

Stick, R., and Hausen, P. (1985). Changes in the nuclear lamina composition during early development of Xenopus laevis. Cell 41, 191-200.

Strelkov, S. V., Schumacher, J., Burkhard, P., Aebi, U., and Herrmann, H. (2004). Crystal structure of the human lamin a coil 2B dimer: Implications for the head-to-tail association of nuclear lamins. J. Mol. Biol. 343, 1067-1080.

Suchanek, M., Radzikowska, A., and Thiele, C. (2005). Photo-leucine and photomethionine allow identification of protein-protein interactions in living cells. Nat. Methods $2,261-267$.

Takata, H., Uchiyama, S., Nakamura, N., Nakashima, S., Kobayashi, S., Sone, T., Kimura, S., Lahmers, S., Granzier, H., Labeit, S., et al. (2007). A comparative proteome analysis of human metaphase chromosomes isolated from two different cell lines reveals a set of conserved chromosome-associated proteins. Genes to Cells 12, 269-284.

Trotta, C.R., Lund, E., Kahan, L., Johnson, A.W., and Dahlberg, J.E. (2003). Coordinated nuclear export of 60s ribosomal subunits and NMD3 in vertebrates. EMBO J. 22, 28412851 .

Turgay, Y., Eibauer, M., Goldman, A.E., Shimi, T., Khayat, M., Ben-Harush, K., Dubrovsky-Gaupp, A., Sapra, K.T., Goldman, R.D., and Medalia, O. (2017). The molecular architecture of lamins in somatic cells. Nature 543, 261-264.

Vartanian, J.P., Henry, M., and Wain-Hobson, S. (1996). Hypermutagenic PCR involving 
all four transitions and a sizeable proportion of transversions. Nucleic Acids Res. 24, $2627-2631$.

Watson, M.L. (1954). Pores in the mammalian nuclear membrane. Biochim. Biophys. Acta $15,475-479$.

Watson, M.L. (1959). Further Observations on the Nuclear Envelope of the Animal Cell. J. Cell Biol. 6, 147-156.

Webster, B.M., Colombi, P., Jäger, J., and Patrick Lusk, C. (2014). Surveillance of nuclear pore complex assembly by ESCRT-III/Vps4. Cell 159, 388-401.

Wen, W., Meinkotht, J.L., Tsien, R.Y., and Taylor, S.S. (1995). Identification of a signal for rapid export of proteins from the nucleus. Cell 82,463-473.

Wente, S.R., Rout, M.P., and Blobel, G. (1992). A New Family of Yeast Nuclear Pore Complex Proteins. 119, 705-723.

Wu, S., Tutuncuoglu, B., Yan, K., Brown, H., Zhang, Y., Tan, D., Gamalinda, M., Yuan, Y., Li, Z., Jakovljevic, J., et al. (2016). Diverse roles of assembly factors revealed by structures of late nuclear pre-60S ribosomes. Nature 534, 133-137.

Wühr, M., Freeman, R.M., Presler, M., Horb, M.E., Peshkin, L., Gygi, S.P., and Kirschner, M.W. (2014). Deep proteomics of the xenopus laevis egg using an mRNA-derived reference database. Curr. Biol. 24, 1467-1475.

$\mathrm{Xu}, \mathrm{S}$. , and Powers, M.A. (2013). In vivo analysis of human nucleoporin repeat domain interactions. Mol. Biol. Cell 24, 1222-1231.

Yamaguchi, A., Iwatani, M., Ogawa, M., Kitano, H., and Matsuyama, M. (2013). In vitro characterization of the RS motif in N-terminal head domain of goldfish germinal vesicle lamin B3 necessary for phosphorylation of the p34cdc2 target serine by SRPK1. FEBS Open Bio 3, 165-176.

Yang, B., Wu, Y.-J., Zhu, M., Fan, S.-B., Lin, J., Zhang, K., Li, S., Chi, H., Li, Y.-X., Chen, H.-F., et al. (2012). Identification of cross-linked peptides from complex samples. Nat. Methods 9, 904-906.

Yao, W., Roser, D., Köhler, A., Bradatsch, B., Baßler, J., and Hurt, E. (2007). Nuclear Export of Ribosomal 60S Subunits by the General mRNA Export Receptor Mex67-Mtr2. 
Mol. Cell 26, 51-62.

Ye, Q., and Worman, H.J. (1996). Interaction between an integral protein of the nuclear envelope inner membrane and human chromodomain proteins homologous to Drosophila HP1. J. Biol. Chem. 271, 14653-14656.

Zeitler, B., and Weis, K. (2004). The FG-repeat asymmetry of the nuclear pore complex is dispensable for bulk nucleocytoplasmic transport in vivo. J. Cell Biol. 167, 583-590. 